\title{
WinGrt, a Windows program for garnet supergroup minerals
}

\author{
Fuat YAVUZ*, Demet Kıran YILDIRIM \\ Department of Geological Engineering, Istanbul Technical University, 34469 Maslak, Istanbul, Turkey; yavuz@itu.edu.tr \\ * Corresponding author
}

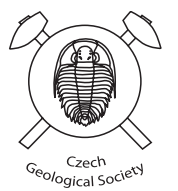

A Microsoft ${ }^{\circledR}$ Visual Basic software, called WinGrt, has been developed to calculate and classify wet-chemical and electron-microprobe garnet supergroup mineral analyses. The program evaluates 33 approved species that belong to the tetragonal henritermierite and isometric bitikleite, schorlomite, garnet and berzeliite groups based on the Commission on New Minerals and Mineral Names (CNMMN) of the International Mineralogical Association (IMA-13) nomenclature scheme. WinGrt also evaluates thirty geothermometers using the $\mathrm{Fe}^{2+}-\mathrm{Mg}$ exchange reactions for garnet-biotite, garnet-clinopyroxene and garnet-orthopyroxene pairs within the application range of greenschist-, amphibolite-, granulite- and eclogite-facies metamorphic rocks. As naturally occurring garnet is potentially a useful provenance indicator, the program calculates end-member molecules from chemical compositions on the basis of different approaches and yields pyrope, almandine, spessartine, grossular, andradite and schorlomite phase on various ternary discrimination diagrams used in provenance studies. The ferric and ferrous iron contents from total $\mathrm{FeO}$ (wt. \%) amount are estimated by stoichiometric constraints. The program allows the users to enter 30 input variables including Sample $\mathrm{No}_{2} \mathrm{SiO}_{2}$, $\mathrm{TiO}_{2}, \mathrm{ZrO}_{2}, \mathrm{HfO}_{2}, \mathrm{Th}_{2} \mathrm{O}, \mathrm{SnO}_{2}, \mathrm{Al}_{2} \mathrm{O}_{3}, \mathrm{Cr}_{2} \mathrm{O}_{3}, \mathrm{~V}_{2} \mathrm{O}_{3}, \mathrm{Fe}_{2} \mathrm{O}_{3}, \mathrm{Mn}_{2} \mathrm{O}_{3}, \mathrm{Sc}_{2} \mathrm{O}_{3}, \mathrm{Y}_{2} \mathrm{O}_{3}+\mathrm{REE}_{2} \mathrm{O}_{3}, \mathrm{FeO}, \mathrm{MgO}, \mathrm{MnO}, \mathrm{ZnO}, \mathrm{CaO}$, $\mathrm{Na}_{2} \mathrm{O}, \mathrm{Li}_{2} \mathrm{O}, \mathrm{P}_{2} \mathrm{O}_{5}, \mathrm{~V}_{2} \mathrm{O}_{5}, \mathrm{Sb}_{2} \mathrm{O}_{5}, \mathrm{As}_{2} \mathrm{O}_{5}, \mathrm{Nb}_{2} \mathrm{O}_{5}, \mathrm{UO}_{3}, \mathrm{Te}_{2} \mathrm{O}_{3}, \mathrm{~F}$ and $\mathrm{H}_{2} \mathrm{O}$ (wt. \%). WinGrt also enables the user to enter the total $\mathrm{REE}_{2} \mathrm{O}_{3}$ (wt. \%) as input values from $\mathrm{La}_{2} \mathrm{O}_{3}$ to $\mathrm{Lu}_{2} \mathrm{O}_{3}$ (wt. \%) of garnet supergroup mineral analyses in program's data edit section. WinGrt enables the user to type or load multiple garnet compositions in the data entry section, to edit and load Microsoft ${ }^{\circledR}$ Excel files in calculating, classifying and naming the garnet species, and to store all the calculated parameters in the Microsoft ${ }^{\mathbb{B}}$ Excel file for further evaluation.

Keywords: garnet, geothermometer, biotite, pyroxene, provenance, software

Received: 19 November 2019; accepted: 28 April 2020; handling editor: J. Konopásek

The online version of this article (doi: 10.3190/jgeosci.303) contains supplementary electronic material

\section{Introduction}

Garnet is one of the best-known minerals on the Earth and a key rock-forming mineral in crystalline rocks of the crust and mantle. The garnet minerals are present in diverse geologic settings and environments from igneous and metamorphic to sedimentary rocks, in remarkable crystal colors and size. In the Earth's crust, garnet is commonly found in metamorphic rocks derived from any protolith, from lower greenschist-facies rocks to ultrahigh-temperature granulites and ultrahigh-pressure eclogites (Baxter et al. 2013; Geiger 2013 and references therein). In igneous rocks, on the other hand, garnet commonly prefers to crystallize in peraluminous granites.

Garnet's density and its relative resistance to surface weathering and diagenetic processes lead to accumulation of this common detrital phase in the heavy mineral fraction of sediments. Consequently, certain garnet compositions including the major end-members such as almandine, pyrope, spessartine, and grossular have been empirically related to specific sources and, therefore, detrital garnets have been increasingly used in sedimentary provenance analysis. A number of binary and ternary discrimination diagrams for garnet have been proposed in the literature (Mange and Morton 2007; Krippner et al. 2014; Suggate and Hall 2014; Tolosana-Delgado et al. 2018). In these diagrams, however, there is no exact and quantitative understanding of what garnet type originates from which host lithology.

Because of the flexible structure, at least 25 essential of total 53 elements were reported in the natural garnets (Geiger 2013; Grew et al. 2013). Garnet's high coordination numbers, as well as relatively high density, low compressibility and increasing stability with increasing pressure make it a petrologically and geochemically important mineral. According to the current IMA-approved nomenclature scheme (Grew et al. 2013), the garnet supergroup includes all minerals isostructural with garnet irrespective of which elements occupy the four atomic sites including three symmetry unique atomic sites $(X, Y$, $Z$ ) and the anionic site $(\phi)$. Hence, the general formula of the garnet supergroup minerals is expressed in the form of $\left\{X_{3}\right\}\left[Y_{2}\right]\left(Z_{3}\right) \phi_{12}$, where $X, Y$, and $Z$ refer to dodecahedral, octahedral and tetrahedral sites, respectively, and $\phi$ contains $\mathrm{O}^{2-}, \mathrm{OH}^{-}$, or $\mathrm{F}^{-}$(Grew et al. 2013). Alternating $Z \phi_{4}$ tetrahedra and $Y \phi_{6}$ octahedra participate of corners to form a three-dimensional framework containing $X \phi_{8}$ triangular dodecahedra (Schingaro et al. 2016). In current 
nomenclature of garnets (Grew et al. 2013), 33 species of the garnet supergroup were accepted, out of which thirty are classified into five groups on the basis of the symmetry and of the total charge of cations at the tetrahedral site. The standard garnet has a cubic symmetry, space group $I a \overline{3} d$. However, two OH-bearing species, henritermierite and holtstamite, have tetragonal symmetry, space group $I 4_{1} / a c d$, with their $X, Z$, and $\phi$ sites split into more symmetrically unique atomic positions.

Chemistry of natural garnet group minerals plays an important role in provenance and geothermobarometry studies that require an accurate estimation of end-member components. Rickwood (1968) proposed a scheme for recasting garnet analyses into 19 endmember molecules with a discussion of the influence of calculation sequence on the end-members allocation. Rickwood's (1968) procedure of recasting garnet analyses into end-member molecules was later implemented by several computer programs and spreadsheet applications (Knowles 1987; Friberg 1989; Muhling and Griffin 1991; Yang 1991; Locock 2008). Although Rickwood's (1968) procedure has been widely used in literature, it was reviewed by Muhling and Griffin (1991) on the account of inappropriate treatment of titanium content. Consequently, a QuickBasic program was developed by Muhling and Griffin (1991) to produce 16 end-members that do not differ significantly from the Rickwood's (1968) procedure for most abundant garnets, but showing variations most notably in the $\mathrm{Cr}, \mathrm{Fe}$ and $\mathrm{Ti}$ allocation. Locock (2008) developed an Excel spreadsheet to recast garnet analyses into 29 possible end-member components comprising 15 mineral species and 14 hypothetical end-members. Grew et al. (2013) proposed an IMA-approved nomenclature scheme for garnet supergroup minerals based on the dominant-valency rule and prepared an Excel spreadsheet to perform calculation, cation allocation, and classification of 32 approved species with group determination. Knierzinger et al. (2019) presented a new interactive MATLAB-based calculation and visualization tool, called TETGAR_C, to assess better spatial and quantitative differentiation trends of detrital garnet minerals including almandine-pyropegrossular-spessartine end-members in the tetrahedral plot system for provenance studies.

In this paper, we present a new computer program, called WinGrt, developed using the Microsoft ${ }^{\circledR}$ Visual Basic programming language to calculate multiple garnets mineral data obtained by both wet-chemical and electronmicroprobe techniques. The program recalculates garnet supergroup mineral analyses based on 8 cations and 12 anions. Calculation and classification of garnet supergroup mineral analyses are carried out according to the current IMA report (Grew et al. 2013). The program is capable of estimating the $\mathrm{Fe}^{3+}$ and $\mathrm{Fe}^{2+}$ contents from microprobe-derived total $\mathrm{FeO}$ (wt. \%) analysis using the stoichiometric constraints proposed by Droop (1987). Using the recalculated garnet analyses, as well as the entered biotite $\left(\mathrm{Al}^{\mathrm{VI}}, \mathrm{Ti}, \mathrm{Fe}^{2+}\right.$, and $\mathrm{Mg}$ ) and pyroxene $\left(\mathrm{Al}_{\text {tot }}, \mathrm{Fe}_{\text {tot }}, \mathrm{Fe}^{2+}, \mathrm{Mg}\right)$ cations (apfu), together with input pressure (kbar) values, WinGrt implements thirty geothermometers for garnet-biotite, garnet-clinopyroxene, and garnet-orthopyroxene pairs. WinGrt allows the user to display garnet supergroup minerals in various ternary classification and variation diagrams by using the Golden Software's Grapher program. When compared to the previously published garnet-related computer programs and Excel spreadsheets, WinGrt provides the users a quick evaluation of multiple garnet analyses for classification as well as geothermometric calculations based on the $\mathrm{Fe}^{2+}-\mathrm{Mg}$ exchange reactions between garnet-biotite, garnet-clinopyroxene and garnet-orthopyroxene pairs and sedimentary provenance analysis using garnet endmembers in ternary classification diagrams.

\section{Program description}

Numerous Excel spreadsheets and executable computer programs have been developed and published for calculation and classification of rock-forming silicate group minerals including amphibole (Yavuz 2007; Locock 2014), mica (Yavuz 2003a, b), pyroxene (Yavuz 2001), garnet (Locock 2008; Grew et al. 2013), tourmaline (Yavuz et al. 2006, 2014), chlorite (Yavuz et al. 2015), epidote (Yavuz and Yildırım 2018) in recent years. However, only a limited number of computer programs appeared in literature for silicate group mineral calculation, classification and thermobarometry estimation (e.g. Putirka 2008; Hora et al. 2013; Yavuz 2013; Lanari et al. 2014; Yavuz et al. 2015; Yavuz and Döner 2017).

WinGrt is a user-friendly compiled program package (14 MB) for garnet supergroup mineral analyses developed for personal computers running in the Microsoft $^{\circledR}$ Windows operating system. The program calculates cations (apfu) from electron-microprobe and wet-chemical garnet analyses and classifies 33 garnet species belonging to five groups including the tetragonal henritermierite group and isometric bitikleite, garnet and berzeliite groups, as well as three species (i.e. katoite, cryolithionite, yafsoanite) that are single representatives of potential groups in which the $Z$ site is vacant or occupied by monovalent or divalent cations (Tab. 1). The current version of program estimates temperatures using ten garnet-biotite (e.g. Ferry and Spear 1978; Hodges and Spear 1982; Perchuk and Lavrent'eva 1983; Bhattacharya et al. 1992), ten garnet-clinopyroxene (Ellis and Green 1979; Krogh 1988; Krogh-Ravna 2000; Nakamura 2009) and ten garnet-orthopyroxene (Raith et al. 1983; Sen and 
Tab. 1 A list of the 33 approved species in the garnet supergroup (from Grew et al. 2013) recognized by the WinGrt program

\begin{tabular}{|c|c|c|c|c|c|}
\hline $\begin{array}{l}\text { NOT SPECIFIED } \\
\text { GROUP } \\
(Z \text { charge }=0-6)\end{array}$ & $\begin{array}{l}\text { HENRITERMIERITE } \\
\text { GROUP } \\
(Z \text { charge }=8)\end{array}$ & $\begin{array}{l}\text { BITIKLEITE } \\
\text { GROUP } \\
(Z \text { charge }=9)\end{array}$ & $\begin{array}{l}\text { SCHORLOMITE } \\
\text { GROUP } \\
(Z \text { charge }=10)\end{array}$ & $\begin{array}{l}\text { GARNET } \\
\text { GROUP } \\
(Z \text { charge }=12)\end{array}$ & $\begin{array}{l}\text { BERZELIITE } \\
\text { GROUP } \\
(Z \text { charge }=15)\end{array}$ \\
\hline $\begin{array}{l}\text { Katoite } \\
\mathrm{Ca}_{3} \mathrm{Al}_{2} \square(\mathrm{OH})_{12}\end{array}$ & $\begin{array}{l}\text { Holtstamite } \\
\mathrm{Ca}_{3} \mathrm{Al}_{2} \mathrm{Si}_{2} \mathrm{I}_{\square} \mathrm{O}_{8}(\mathrm{OH})_{4}\end{array}$ & $\begin{array}{l}\text { Bitikleite } \\
\mathrm{Ca}_{3} \mathrm{Sb}^{5+} \mathrm{Sn}^{4+} \mathrm{Al}_{3} \mathrm{O}_{12}\end{array}$ & $\begin{array}{l}\text { Kimzeyite } \\
\mathrm{Ca}_{3} \mathrm{Zr}_{2} \mathrm{SiAl}_{2} \mathrm{O}_{12}\end{array}$ & $\begin{array}{l}\text { Menzerite-(Y) } \\
\mathrm{Y}_{2} \mathrm{CaMg}_{2} \mathrm{Si}_{3} \mathrm{O}_{12}\end{array}$ & $\begin{array}{l}\text { Schäferite } \\
\mathrm{Ca}_{2} \mathrm{NaMg}_{2} \mathrm{~V}_{3+}^{5+} \mathrm{O}_{12}\end{array}$ \\
\hline $\begin{array}{l}\text { Cryolithionite } \\
\mathrm{Na}_{3} \mathrm{Al}_{2} \mathrm{Li}_{3} \mathrm{~F}_{12}\end{array}$ & $\begin{array}{l}\text { Henritermierite } \\
\mathrm{Ca}_{3} \mathrm{Mn}^{3+}{ }_{2} \mathrm{Si}_{2} \mathrm{I} \square \mathrm{O}_{8}(\mathrm{OH})_{4}\end{array}$ & $\begin{array}{l}\text { Usturite } \\
\mathrm{Ca}_{3} \mathrm{Sb}^{5+} \mathrm{ZrFe}_{3}^{3+} \mathrm{O}_{12}\end{array}$ & $\begin{array}{l}\text { Irinarassite } \\
\mathrm{Ca}_{3} \mathrm{Sn}_{2}^{4+} \mathrm{SiAl}_{2} \mathrm{O}_{12}\end{array}$ & $\begin{array}{l}\text { Pyrope } \\
\mathrm{Mg}_{3} \mathrm{Al}_{2} \mathrm{Si}_{3} \mathrm{O}_{12}\end{array}$ & $\begin{array}{l}\text { Palenzonaite } \\
\mathrm{Ca}_{2} \mathrm{NaMn}_{2}^{2+} \mathrm{V}_{2}^{5+}{ }_{3} \mathrm{O}_{12}\end{array}$ \\
\hline \multirow[t]{12}{*}{$\mathrm{Ca}_{3} \mathrm{Te}^{6+}{ }_{2} \mathrm{Zn}_{3} \mathrm{O}_{12}$} & & $\begin{array}{l}\text { Dzhuluite } \\
\mathrm{Ca}_{3} \mathrm{Sb}^{5+} \mathrm{Sn}^{4+} \mathrm{Fe}^{3+}{ }_{3} \mathrm{O}_{12}\end{array}$ & $\begin{array}{l}\text { Schorlomite } \\
\mathrm{Ca}_{3} \mathrm{Ti}_{2} \mathrm{SiFe}^{3+}{ }_{2} \mathrm{O}_{12}\end{array}$ & $\begin{array}{l}\text { Grossular } \\
\mathrm{Ca}_{3} \mathrm{Al}_{2} \mathrm{Si}_{3} \mathrm{O}_{12} \\
\end{array}$ & $\begin{array}{l}\text { Berzeliite } \\
\mathrm{Ca}_{2} \mathrm{NaMg}_{2} \mathrm{As}^{5+}{ }_{3} \mathrm{O}_{12}\end{array}$ \\
\hline & & $\begin{array}{l}\text { Elbrusite } \\
\mathrm{Ca}_{3} \mathrm{U}^{6+}{ }_{0.5} \mathrm{Zr}_{1.5} \mathrm{Fe}_{3}^{3+} \mathrm{O}_{12}\end{array}$ & $\begin{array}{l}\text { Kerimasite } \\
\mathrm{Ca}_{3} \mathrm{Zr}_{2} \mathrm{SiFe}_{2}^{3+} \mathrm{O}_{12} \\
\end{array}$ & $\begin{array}{l}\text { Spessartine } \\
\mathrm{Mn}_{3}^{2+} \mathrm{Al}_{2} \mathrm{Si}_{3} \mathrm{O}_{12}\end{array}$ & $\begin{array}{l}\text { Maganberzeliite } \\
\mathrm{Ca}_{2} \mathrm{NaMn}_{2}^{2+} \mathrm{As}_{2}^{5+} \mathrm{O}_{12}\end{array}$ \\
\hline & & & $\begin{array}{l}\text { Toturite } \\
\mathrm{Ca}_{3} \mathrm{Sn}_{2}^{4+} \mathrm{SiFe}_{2}^{3+} \mathrm{O}_{12}\end{array}$ & $\begin{array}{l}\text { Almandine } \\
\mathrm{Fe}^{2+}{ }_{3} \mathrm{Al}_{2} \mathrm{Si}_{3} \mathrm{O}_{12}\end{array}$ & \\
\hline & & & $\begin{array}{l}\text { Hutcheonite }^{\dagger} \\
\mathrm{Ca}_{3} \mathrm{Ti}_{2}\left(\mathrm{SiAl}_{2}\right) \mathrm{O}_{12}\end{array}$ & $\begin{array}{l}\text { Eringaite } \\
\mathrm{Ca}_{3} \mathrm{Sc}_{2} \mathrm{Si}_{3} \mathrm{O}_{12}\end{array}$ & \\
\hline & & & & $\begin{array}{l}\text { Goldmanite } \\
\mathrm{Ca}_{3} \mathrm{~V}^{3+}{ }_{2} \mathrm{Si}_{3} \mathrm{O}_{12}\end{array}$ & \\
\hline & & & & $\begin{array}{l}\text { Momoiite } \\
\mathrm{Mn}_{3}^{2+} \mathrm{V}_{3}^{3+} \mathrm{Si}_{3} \mathrm{O}_{12}\end{array}$ & \\
\hline & & & & $\begin{array}{l}\text { Knorringite } \\
\mathrm{Mg}_{3} \mathrm{Cr}^{3+}{ }_{2} \mathrm{Si}_{3} \mathrm{O}_{12}\end{array}$ & \\
\hline & & & & $\begin{array}{l}\text { Uvarovite } \\
\mathrm{Ca}_{3} \mathrm{Cr}^{3+} \mathrm{Si}_{3} \mathrm{O}_{12}\end{array}$ & \\
\hline & & & & $\begin{array}{l}\text { Andradite } \\
\mathrm{Ca}_{3} \mathrm{Fe}^{3+}{ }_{2} \mathrm{Si}_{3} \mathrm{O}_{12}\end{array}$ & \\
\hline & & & & $\begin{array}{l}\text { Calderite } \\
\mathrm{Mn}_{3}^{2+} \mathrm{Fe}^{3+}{ }_{2} \mathrm{Si}_{3} \mathrm{O}_{12}\end{array}$ & \\
\hline & & & & $\begin{array}{l}\text { Majorite } \\
\mathrm{Mg}_{3} \mathrm{SiMgSi}_{3} \mathrm{O}_{12}\end{array}$ & \\
\hline & & & & $\begin{array}{l}\text { Morimotoite } \\
\mathrm{Ca}_{3} \mathrm{TiFe}^{2+} \mathrm{Si}_{3} \mathrm{O}_{12}\end{array}$ & \\
\hline
\end{tabular}

${ }^{\dagger}$ New garnet species approved by the IMA (IMA 2013-029) later than the subcommittee report by Grew et al. (2013)

Bhattacharya 1984; Perchuk and Lavrent'eva 1990; Lal 1993) geothermometers based on the exchange reactions between $\mathrm{Fe}^{2+}$ and $\mathrm{Mg}$.

Heavy minerals have been used in a number of provenance studies to discriminate and identify the source areas. Garnets are one of the commonly employed minerals due to their occurrence in a wide range of rocks, relative stability under both weathering and diagenetic conditions, as well as a wide range of major-element compositions. WinGrt calculates end-member molecules such as pyrope, almandine, spessartine, grossular, andradite, and schorlomite and allows the users to display them on various ternary classification diagrams in sedimentary provenance studies. A list of the calculation steps in the Calculation Screen and in an Excel output of the WinGrt is given in Tab. 2. Current version presents eight ternary garnet classification and nine garnet provenance-related ternary plots. These plots are displayed by the Golden Software's Grapher program by selecting diagram types from the pull-down menu of Graph in the Calculation Screen of WinGrt.

\subsection{Site allocation of cations in the garnet supergroup and determination of garnet species}

Classification of a rock-forming silicate mineral species is based on the chemical composition of an analyzed sample obtained by wet-chemical or electron-microprobe techniques. Proper site allocation of rock-forming minerals is an important issue, commonly demanding single-crystal, or Rietveld structure refinement using X-ray or neutron diffraction methods. Although most researchers have only electron-microprobe analyses, which provide no direct evidence of valence state, spectroscopic data are also required for unambiguous site assignment, especially when constituents could be present in more than one valence state. The general formula for the garnet supergroup minerals is expressed in the form of $\left\{X_{3}\right\}\left[Y_{2}\right]\left(Z_{3}\right) \phi_{12}$, where $X, Y$, and $Z$ refer to dodecahedral, octahedral, and tetrahedral sites, respectively, and $\phi$ is $\mathrm{O}, \mathrm{OH}$, or F (Grew et al. 2013). The site occupancies used in the 
garnet supergroup general formula by WinGrt program are as follows:

$\{X\}=\left(\mathrm{Th}^{4+}, \mathrm{Y}^{3+}+\mathrm{REE}^{3+}, \mathrm{Ca}^{2+}, \mathrm{Na}^{+}\right), \mathrm{Mn}^{2+}, \mathrm{Fe}^{2+}, \mathrm{Mg}^{2+}$; $[Y]=\mathrm{Zr}^{4+}, \mathrm{Hf}^{4+}, \mathrm{Sn}^{4+}, \mathrm{U}^{6+}, \mathrm{Te}^{6+}, \mathrm{Sb}^{5+}, \mathrm{Nb}^{5+}, \mathrm{Sc}^{3+}, \mathrm{Cr}^{3+}, \mathrm{V}^{3+}$, $\mathrm{Si}^{4+}, \mathrm{Ti}^{4+}, \mathrm{Al}^{3+}, \mathrm{Mn}^{3+}, \mathrm{Fe}^{3+}, \mathrm{Mg}^{2+}, \mathrm{Fe}^{2+}, \mathrm{Mn}^{2+}$;

$(Z)=\mathrm{As}^{5+}, \mathrm{V}^{5+}, \mathrm{P}^{5+}, \mathrm{Li}^{+}, \mathrm{Zn}^{2+}, \mathrm{Si}^{4+}, \mathrm{Al}^{3+}, \mathrm{Fe}^{3+}, \mathrm{Fe}^{2+}, \mathrm{Ti}^{4+}$, $\mathrm{H}_{4}^{+}$, vacancy $\left({ }^{Z} \square\right)$;

$\phi=\mathrm{O}^{2-}, \mathrm{OH}^{-}, \mathrm{F}^{-}$.

Several elements that rarely exceed $1 \mathrm{wt} . \%$ in natural garnets (e.g. B, S, K, Ni, or $\mathrm{Sr}$ ) as well as $\mathrm{Ge}, \mathrm{Ga}$ and $\mathrm{Pb}$ that appear in significant amounts, but with rare occurrence, have been excluded from program's data entry and calculation steps. The following calculation and site allocation procedures were implemented in nomenclature of the garnet supergroup minerals by WinGrt program:

(1) Calculation of formula from the chemical analysis is performed based on 8 cations and 12 anions. Ferric and ferrous iron separation from electron-microprobe analysis, as well as the proportion of $\mathrm{Mn}^{2+}$ and $\mathrm{Mn}^{3+}$, are assessed using the Droop's (1987) method. If quantitative $\mathrm{F}$ (wt. \%) and $\mathrm{H}_{2} \mathrm{O}$ (wt. \%) data are available, then ${ }_{\square}=1 / 4 \mathrm{~F}+1 / 4 \mathrm{H}$.

(2) $\mathrm{Li}^{+}, \mathrm{Zn}^{2+}, \mathrm{P}^{5+}, \mathrm{As}^{5+}$ and $\mathrm{V}^{5+}$ are allocated to the $Z$ site. If $\mathrm{Li}^{+}<1 / 4 \mathrm{~F}^{-}$, then sufficient vacancies are assumed to make up the deficit.

(3) $\mathrm{Si}^{4+}$ is first allocated to the $Z$ site. If $\mathrm{Si}(a p f u)>3$ (including vacancies), then excess $\mathrm{Si}$ is added to the $Y$ site.

(4) $\mathrm{Al}^{3+}$ is first allocated to the $Z$ site to bring total to 3 (apfu), then to the $Y$ site.

(5) $\mathrm{Fe}^{3+}$ is first allocated to the $Z$ site to bring total to 3 (apfu), then to the $Y$ site.

(6) $\mathrm{Ca}^{2+}, \mathrm{Na}^{+}, \mathrm{Y}^{3+}, \mathrm{REE}^{3+}$, and $\mathrm{Th}^{4+}$ are allocated to the $X$ site.

(7) $\mathrm{Al}^{3+}$ (remaining aluminum content at the $Z$ site; i.e. $\mathrm{Al}_{\text {tot }}{ }^{Z} \mathrm{Al}$ ), $\mathrm{Sc}^{3+}, \mathrm{Ti}^{4+}, \mathrm{V}^{3+}, \mathrm{Cr}^{3+}, \mathrm{Mn}^{3+}, \mathrm{Fe}^{3+}$ (remaining ferric iron content at the $Z$ site; i.e. $\mathrm{Fe}^{3+}{ }_{\text {tot }}{ }^{Z} \mathrm{Fe}^{3+}$ ), $\mathrm{Zr}^{4+}, \mathrm{Hf}^{4+}, \mathrm{Nb}^{5+}, \mathrm{Sn}^{4+}, \mathrm{Sb}^{5+}, \mathrm{Te}^{6+}$, and $\mathrm{U}^{6+}$ are allocated to the $Y$ site. If the $Z$ site is still $<3$ (apfu), then $\mathrm{Fe}^{2+}$ is added to bring the $Z$ site total to 3 (apfu). If the content of $Y$ site exceeds 2 ( $a p f u$ ), and the $Z$ site is $<3$ (apfu), then $\mathrm{Ti}^{4+}$ is moved to the $Z$ site to bring the $Z$ site total to 3 (apfu).

(8) $\mathrm{Mg}^{2+}$ is first allocated to the $Y$ site to bring total to 2 (apfu), then remaining to the $X$ site.

(9) $\mathrm{Fe}^{2+}$ (remaining ferrous iron content at the $Z$ site; i.e. $\left.\mathrm{Fe}^{2+}{ }_{\text {tot }}{ }^{Z} \mathrm{Fe}^{2+}\right)$ is first allocated to the $Y$ site to bring total to 2 (apfu), then to the $X$ site.

(10) $\mathrm{Mn}^{2+}$ is first allocated to the $Y$ site to bring total to 2 (apfu), then to the $X$ site.

According to Grew et al. (2013), the site allocation procedure listed above fails to differentiate holtstamite, a member of the hentermierite group, from grossular of the garnet group, which would require additional information such as optical properties or crystallographic data. Following the classification procedures by Henry et al. (2011) for nomenclature of the tourmaline-supergroup minerals, the dominant valence is determined for each site by summing the ions for each valence, and then the dominant cation identified. The dominant ion for each valence determines the species belonging to each garnet group. Detailed nomenclature scheme on determination of the garnet supergroup species was explained in the current IMA-approved report (Grew et al. 2013).

\subsection{Data entry of analyses}

Upon successful installation of WinGrt, the start-up screen with various pull-down menus and equivalent shortcuts appears. The program allows the user to type garnet analyses (Fig. 1a; wt. \%) and selected cation values of biotite, clinopyroxene and orthopyroxene (Fig. 1b; apfu) by clicking the New icon on the tool bar, selecting the New File from the pull-down menu of File option or pressing the $\mathrm{Ctrl}+\mathrm{N}$ keys. Entered biotite, clinopyroxene, and orthopyroxene cations $(a p f u)$ with input pressure (kbar) values are used by program for estimations of garnet-biotite, garnet-clinopyroxene, and garnet-orthopyroxene geothermometers. In the New File, Data Entry Screen, and Calculation Screen, these parameters are highlighted by the ice blue, moon green and faded pink colors, respectively. The standard 30 variables (wt. \%) are used by WinGrt for calculation and classification of garnet supergroup mineral analyses in the following order:

Sample No, $\mathrm{SiO}_{2}, \mathrm{TiO}_{2}, \mathrm{ZrO}_{2}, \mathrm{HfO}_{2}, \mathrm{ThO}_{2}, \mathrm{SnO}_{2}$, $\mathrm{Al}_{2} \mathrm{O}_{3}, \mathrm{Cr}_{2} \mathrm{O}_{3}, \mathrm{~V}_{2} \mathrm{O}_{3}, \mathrm{Fe}_{2} \mathrm{O}_{3}, \mathrm{Mn}_{2} \mathrm{O}_{3}, \mathrm{Sc}_{2} \mathrm{O}_{3}, \mathrm{Y}_{2} \mathrm{O}_{3}+\mathrm{REE}_{2} \mathrm{O}_{3}$, $\mathrm{FeO}, \mathrm{MgO}, \mathrm{MnO}, \mathrm{ZnO}, \mathrm{CaO}, \mathrm{Na}_{2} \mathrm{O}, \mathrm{Li}_{2} \mathrm{O}, \mathrm{P}_{2} \mathrm{O}_{5}, \mathrm{~V}_{2} \mathrm{O}_{5}$, $\mathrm{Sb}_{2} \mathrm{O}_{5}, \mathrm{As}_{2} \mathrm{O}_{5}, \mathrm{Nb}_{2} \mathrm{O}_{5}, \mathrm{UO}_{3}, \mathrm{TeO}_{3}, \mathrm{~F}$ and $\mathrm{H}_{2} \mathrm{O}$ (wt. \%). However, rare earth element oxides $\left(\mathrm{REE}_{2} \mathrm{O}_{3}\right)$ analyses from $\mathrm{La}_{2} \mathrm{O}_{3}$ to $\mathrm{Lu}_{2} \mathrm{O}_{3}$ (wt. \%) can be typed separately in the Data Entry Screen section of program.

Garnet analyses entered in an Excel file (". xls" or ".xIsx") in the above order together with biotite, clinopyroxene, orthopyroxene cations (apfu) and pressure (kbar) values can be loaded into the program's Data Entry Screen by clicking the Open Excel File option from the File pull-down menu. By selecting the Edit Excel File option from the File pull-down menu, these can be typed in a blank Excel file (i.e. MyGarnet), stored under a different file name, and then loaded into the program's Data Entry Screen by clicking the Open Excel File option from the File pull-down menu. Additional information about data entry or similar topics can be accessed by pressing the F1 function key to display the WinGrt. chm file on the screen. 


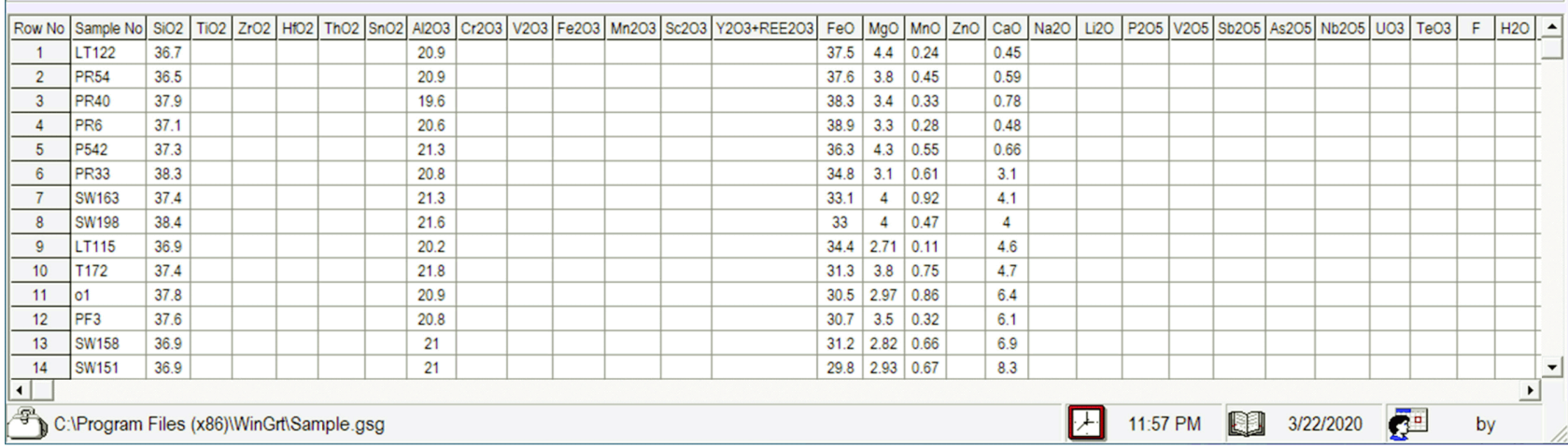

WinGrt

File Edit Calculate About Help

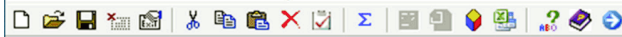

A Windows Program for Garnet Supergroup Minerals

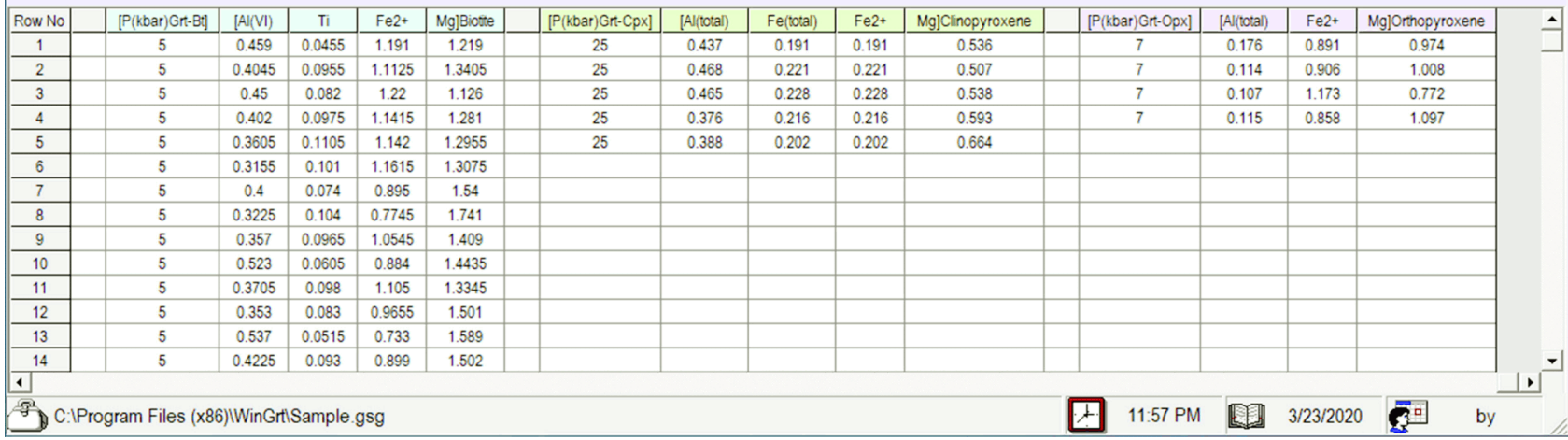

Fig. 1 Screenshots of the WinGrt Data Entry Screen window. a - Editing garnet supergroup mineral analyses (wt. \%). b - Recalculated input biotite, clinopyroxene and orthopyroxene cations $(a p f u)$ for geothermometer estimations.

\section{Worked examples}

Using the selected data set from literature, the following examples show how WinGrt can be used in calculation and classification of the garnet supergroup minerals (see Electronic Supplementary Material, ESM 1), as well as in estimation of temperatures by garnet-biotite, garnet-clinopyroxene and garnet-orthopyroxene geothermometers. Once the previously typed or loaded garnet analyses are processed by clicking the Calculate icon (i.e. $\sum$ ) in the Data Entry Section of the program, all output and input parameters are displayed in columns 1-187 (see Tab. 2) of the Calculation Screen. Pressing the Ctrl + F keys or clicking the Open File to Calculate option from the Calculate menu also executes the data processing for a selected data file with the extension of ". gs g". By clicking the Send results to Excel file icon in the Calculation Screen, all calculations can be stored in an Excel file (Output.xlsx) and then displayed by clicking the Open and edit Excel file icon.

Validity of WinGrt outputs has been tested (Tab. 3) with representative garnet supergroup mineral analyses selected from literature (e.g. Munno et al. 1980; Galuskina et al. 2010; Nagashima and Armbruster 2012; Galuskina et al. 2013; Grew et al. 2013; Antao 2014; $\mathrm{Ma}$ et al. 2014; Antao and Cruickshank 2018). WinGrt calculates cations $(a p f u)$ of garnet supergroup mineral analyses (see rows 38-68 in Fig. 2a) and then allocates the recalculated values into the $Z, Y$, and $X$ sites, respectively (see columns $70-90$ in Fig. $2 b$ and $91-111$ in Fig. 2c; rows $28-63$ in Tab. 3). Anion contents and total of dominant monovalent, divalent, trivalent, tetravalent, pentavalent and hexavalent cations (apfu) in the $X, Y$ and $Z$ sites are listed in columns 113-116 and 118-135 of the Calculation Screen window (see Fig. 2d). Dominant charge formula and type with garnet group names 
Tab. 2 Description of column numbers in the Calculation Screen window of WinGrt program and an output Excel file

\begin{tabular}{|c|c|c|}
\hline Row & Explanation & Column numbers \\
\hline 1 & Major oxide garnet supergroup mineral analyses (wt. \%) & $1-31$ \\
\hline 2 & Blank & 32 \\
\hline 3 & Recalculated $\mathrm{FeO}, \mathrm{Fe}_{2} \mathrm{O}_{3}, \mathrm{MnO}$ and $\mathrm{Mn}_{2} \mathrm{O}_{3}$ (wt. \%) contents based on the stoichiometric constraints & $33-36$ \\
\hline 4 & Blank & 37 \\
\hline 5 & Recalculated cations of garnet supergroup mineral analyses (apfu) & $38-68$ \\
\hline 6 & Blank & 69 \\
\hline 7 & Cations allocation at the $Z$ site & $70-82$ \\
\hline 8 & Blank & 83 \\
\hline 9 & Cations allocation at the $Y$ site & $84-102$ \\
\hline 10 & Blank & 103 \\
\hline 11 & Cations allocation at the $X$ site & $104-111$ \\
\hline 12 & Blank & 112 \\
\hline 13 & Anions allocation at the $\varphi$ site & $113-116$ \\
\hline 14 & Blank & 117 \\
\hline 15 & Sums of homovalent cations by site and sums of monovalent and divalent anions & $118-135$ \\
\hline 16 & Blank & 136 \\
\hline 17 & Dominant charge formula & 137 \\
\hline 18 & Dominant formula type & 138 \\
\hline 19 & Garnet group & 139 \\
\hline 20 & Garnet species & 140 \\
\hline 21 & Blank & 141 \\
\hline 22 & Input $P$ (kbar) and $\mathrm{Al}^{\mathrm{vI}}, \mathrm{Ti}, \mathrm{Fe}^{2+}$ and $\mathrm{Mg}(a p f u)$ values of $\mathrm{Bt}$ for garnet-biotite geothermometers & $142-146$ \\
\hline 23 & Garnet-biotite geothermometers & $147-156$ \\
\hline 24 & Blank & 157 \\
\hline 25 & Input $P(\mathrm{kbar})$ and $\mathrm{Al}_{\text {tot }}, \mathrm{Ti}_{1} \mathrm{Fe}_{\text {tot }}$ and $\mathrm{Mg}(a p f u)$ values of $\mathrm{Cpx}$ for garnet-clinopyroxene geothermometers & $158-162$ \\
\hline 26 & Garnet-clinopyroxene geothermometers & $163-172$ \\
\hline 27 & Blank & 173 \\
\hline 28 & Input $P$ (kbar) and $\mathrm{Al}_{\text {tot }}, \mathrm{Fe}^{2+}$ and $\mathrm{Mg}(a p f u)$ values of Opx for garnet-orthopyroxene geothermometers & $174-177$ \\
\hline 29 & Garnet-orthopyroxene geothermometers & $178-187$ \\
\hline
\end{tabular}

Note: $a p f u=$ atoms per formula unit; $\mathrm{Bt}=$ biotite, $\mathrm{Cpx}=$ clinopyroxene, $\mathrm{Opx}=$ orthopyroxene

and species according to the nomenclature scheme by Grew et al. (2013) are presented in columns 137-140 of the Calculation Screen window (Fig. 3; see rows 68-70 in Tab. 3) for selected mineral analyses from literature. WinGrt calculates $\mathrm{Fe}^{2+}-\mathrm{Mg}$ exchange reaction geothermometers only for a garnet analysis that belongs to the garnet group including almandine, pyrope, spessartine and grossular species. Garnet-biotite geothermometers with input biotite cations (apfu) and pressure (kbar) values are presented in columns $142-156$ of the Calculation Screen window (Fig. 4a). On the other hand, garnetclinopyroxene and garnet-orthopyroxene geothermometer estimations by WinGrt are listed with input clino- and orthopyroxene cations (apfu) and pressure (kbar) values in columns 158-172 (Fig. 4b) and 174-187 (Fig. 4c) of the Calculation Screen window, respectively.

\subsection{Geothermometry}

Estimating the temperature at which minerals crystallized in rocks is an important goal for petrologists and geochemists. Hence, various methods to estimate the $P-T$ conditions of geologic environments have been developed based on laboratory experiments, thermodynamic calculations, and electron-microprobe mineral analyses. Geothermometers that are based on the exchange reactions (e.g. $\mathrm{Fe}^{2+}$ and $\mathrm{Mg}$ ) between different sites in a single mineral or between mineral pairs are widely used in metamorphic rocks involving e.g. olivine, garnet, clinopyroxene, orthopyroxene, spinel, ilmenite, cordierite, biotite, phengite, chlorite and hornblende (Bucher and Grapes 2011). Current version of WinGrt offers the most widely used $\mathrm{Fe}^{2+}-\mathrm{Mg}$ exchange calibrations in garnet-biotite, garnet-clinopyroxene and garnet-orthopyroxene pairs within the application range of greenschist-, amphibolite-, granulite- and eclogite-facies metamorphic rocks.

\subsubsection{Garnet-biotite geothermometers}

When compared to the other all $\mathrm{Fe}^{2+}-\mathrm{Mg}$ exchange geothermometers, the garnet-biotite is the most popular one due to its applicability to a large variety of rocks and covering a broad range of metamorphic grades from amphibolite- to granulite-facies (see row 21 in Tab. 4). The 
Tab. 3 Chemical compositions of selected garnet supergroup minerals recalculated and classified by WinGrt

\begin{tabular}{|c|c|c|c|c|c|c|c|c|c|c|c|}
\hline \multicolumn{2}{|c|}{ Row } & \multirow{2}{*}{$\begin{array}{r}\text { SG1 } \\
30.64\end{array}$} & \multirow{2}{*}{$\begin{array}{r}\text { SG2 } \\
31.80\end{array}$} & \multirow{2}{*}{$\begin{array}{r}\text { SG3 } \\
36.60\end{array}$} & \multirow{2}{*}{$\begin{array}{c}\text { SG4 } \\
16.63\end{array}$} & \multirow{2}{*}{$\begin{array}{c}\text { SG5 } \\
25.72\end{array}$} & \multirow{2}{*}{$\begin{array}{c}\text { SG6 } \\
20.90\end{array}$} & \multirow{2}{*}{$\begin{array}{c}\text { SG7 } \\
0.28\end{array}$} & \multirow{2}{*}{$\begin{array}{c}\text { SG8 } \\
0.28\end{array}$} & \multirow{2}{*}{$\begin{array}{c}\text { SG9 } \\
24.64\end{array}$} & \multirow{2}{*}{$\begin{array}{c}\text { SG10 } \\
0.73\end{array}$} \\
\hline 1 & $\mathrm{SiO}_{2}$ & & & & & & & & & & \\
\hline 2 & $\mathrm{TiO}_{2}$ & 1.10 & 1.76 & 0.00 & 6.95 & 16.00 & 25.30 & 2.66 & 2.62 & 0.00 & 0.03 \\
\hline 3 & $\mathrm{ZrO}_{2}$ & 0.00 & 0.12 & 0.00 & 27.38 & 2.98 & 0.00 & 0.28 & 4.21 & 0.00 & 0.00 \\
\hline 4 & $\mathrm{HfO}_{2}$ & 0.00 & 0.00 & 0.00 & 0.00 & 0.00 & 0.00 & 0.01 & 0.00 & 0.00 & 0.00 \\
\hline 5 & $\mathrm{SnO}_{2}$ & 0.00 & 0.00 & 0.00 & 0.00 & 0.00 & 0.00 & 16.65 & 16.70 & 0.00 & 0.00 \\
\hline 6 & $\mathrm{Al}_{2} \mathrm{O}_{3}$ & 4.87 & 11.20 & 0.00 & 9.37 & 0.72 & 15.70 & 11.65 & 6.17 & 0.41 & 0.00 \\
\hline 7 & $\mathrm{Cr}_{2} \mathrm{O}_{3}$ & 0.04 & 0.00 & 0.00 & 0.00 & 0.01 & 0.000 & 0.02 & 0.00 & 0.00 & 0.00 \\
\hline 8 & $\mathrm{~V}_{2} \mathrm{O}_{3}$ & 0.17 & 0.21 & 0.00 & 0.00 & 0.55 & 0.50 & 0.00 & 0.00 & 0.00 & 0.00 \\
\hline 9 & $\mathrm{Fe}_{2} \mathrm{O}_{3}$ & 8.38 & 0.00 & 0.00 & 0.00 & 18.14 & 0.00 & 14.38 & 19.82 & 0.33 & 0.00 \\
\hline 10 & $\mathrm{Mn}_{2} \mathrm{O}_{3}$ & 0.00 & 0.00 & 0.00 & 0.00 & 0.000 & 0.00 & 0.00 & 0.00 & 31.69 & 0.00 \\
\hline 11 & $\mathrm{Sc}_{2} \mathrm{O}_{3}$ & 0.17 & 0.00 & 0.00 & 0.00 & 0.00 & 0.00 & 0.00 & 0.05 & 0.00 & 0.00 \\
\hline 12 & $\mathrm{Y}_{2} \mathrm{O}_{3}+\mathrm{REE}_{2} \mathrm{O}_{3}$ & 25.32 & 0.00 & 0.00 & 0.00 & 0.00 & 0.00 & 0.00 & 0.00 & 0.00 & 0.00 \\
\hline 13 & $\mathrm{FeO}$ & 11.82 & 13.50 & 27.26 & 6.43 & 2.21 & 0.70 & 1.11 & 2.20 & 0.00 & 0.06 \\
\hline 14 & $\mathrm{MgO}$ & 3.98 & 0.18 & 0.00 & 3.32 & 1.49 & 2.10 & 0.22 & 0.02 & 0.01 & 11.69 \\
\hline 15 & $\mathrm{MnO}$ & 0.85 & 0.12 & 0.00 & 0.00 & 0.47 & 0.000 & 0.03 & 0.00 & 0.00 & 3.51 \\
\hline 16 & $\mathrm{CaO}$ & 13.92 & 35.80 & 33.90 & 30.25 & 31.63 & 34.60 & 25.38 & 23.86 & 34.77 & 20.63 \\
\hline 17 & $\mathrm{Na}_{2} \mathrm{O}$ & 0.00 & 0.07 & 0.00 & 0.00 & 0.22 & 0.00 & 0.00 & 0.00 & 0.00 & 4.82 \\
\hline 18 & $\mathrm{P}_{2} \mathrm{O}_{5}$ & 0.00 & 0.00 & 0.00 & 0.00 & 0.00 & 0.00 & 0.00 & 0.00 & 0.00 & 0.25 \\
\hline 19 & $\mathrm{~V}_{2} \mathrm{O}_{5}$ & 0.00 & 0.00 & 0.00 & 0.00 & 0.00 & 0.00 & 0.00 & 0.00 & 0.00 & 0.33 \\
\hline 20 & $\mathrm{Sb}_{2} \mathrm{O}_{5}$ & 0.00 & 0.00 & 0.00 & 0.00 & 0.00 & 0.00 & 25.98 & 16.73 & 0.00 & 0.00 \\
\hline 21 & $\mathrm{As}_{2} \mathrm{O}_{5}$ & 0.00 & 0.00 & 0.00 & 0.00 & 0.00 & 0.00 & 0.00 & 0.000 & 0.00 & 57.40 \\
\hline 22 & $\mathrm{Nb}_{2} \mathrm{O}_{5}$ & 0.00 & 0.00 & 0.00 & 0.00 & 0.00 & 0.00 & 0.07 & 0.08 & 0.00 & 0.00 \\
\hline 23 & $\mathrm{UO}_{3}$ & 0.00 & 0.00 & 0.00 & 0.00 & 0.00 & 0.00 & 0.05 & 6.30 & 0.00 & 0.00 \\
\hline 24 & $\mathrm{~F}$ & 0.00 & 3.13 & 0.00 & 0.00 & 0.00 & 0.00 & 0.00 & 0.00 & 0.00 & 0.00 \\
\hline 25 & $\mathrm{O}=\mathrm{F}$ & 0.00 & -1.318 & 0.00 & 0.00 & 0.00 & 0.00 & 0.00 & 0.00 & 0.00 & 0.00 \\
\hline 26 & $\mathrm{H}_{2} \mathrm{O}$ & 0.00 & 1.93 & 0.00 & 0.00 & 0.00 & 0.00 & 0.00 & 0.00 & 7.57 & 0.00 \\
\hline 27 & $\sum($ wt. \%) & 101.26 & 98.502 & 97.60 & 100.33 & 100.14 & 99.80 & 98.79 & 99.04 & 99.42 & 99.45 \\
\hline 28 & $\mathrm{Si}$ & 2.762 & 2.485 & 3.000 & 1.507 & 2.214 & 1.680 & 0.031 & 0.033 & 1.981 & 0.070 \\
\hline 29 & $\mathrm{Ti}$ & 0.000 & 0.000 & 0.000 & 0.005 & 0.000 & 0.000 & 0.129 & 0.149 & 0.000 & 0.000 \\
\hline 30 & $\mathrm{Al}$ & 0.238 & 0.071 & 0.000 & 1.001 & 0.073 & 1.320 & 1.530 & 0.853 & 0.000 & 0.000 \\
\hline 31 & $\mathrm{Fe}^{3+}$ & 0.000 & 0.000 & 0.000 & 0.487 & 0.713 & 0.000 & 1.206 & 1.750 & 0.000 & 0.000 \\
\hline 32 & As & 0.000 & 0.000 & 0.000 & 0.000 & 0.000 & 0.000 & 0.000 & 0.000 & 0.000 & 2.885 \\
\hline 33 & $\mathrm{~V}^{5+}$ & 0.000 & 0.000 & 0.000 & 0.000 & 0.000 & 0.000 & 0.001 & 0.000 & 0.000 & 0.021 \\
\hline 34 & $\mathrm{P}$ & 0.000 & 0.000 & 0.000 & 0.000 & 0.000 & 0.000 & 0.000 & 0.000 & 0.000 & 0.020 \\
\hline 35 & $\mathrm{Fe}^{2+}$ & 0.000 & 0.000 & 0.000 & 0.000 & 0.000 & 0.000 & 0.103 & 0.216 & 0.000 & 0.004 \\
\hline 36 & $\mathrm{H}_{4}$ & 0.000 & 0.251 & 0.000 & 0.000 & 0.000 & 0.000 & 0.000 & 0.000 & 1.019 & 0.000 \\
\hline 37 & vacancy & 0.000 & 0.193 & 0.000 & 0.000 & 0.000 & 0.000 & 0.000 & 0.000 & 0.000 & 0.000 \\
\hline 38 & $\sum Z$ site & 3.000 & 3.000 & 3.000 & 3.000 & 3.000 & 3.000 & 3.000 & 3.000 & 3.000 & 3.000 \\
\hline 39 & $\mathrm{Si}$ & 0.000 & 0.000 & 0.059 & 0.000 & 0.000 & 0.000 & 0.000 & 0.000 & 0.007 & 0.000 \\
\hline 40 & $\mathrm{Ti}$ & 0.075 & 0.103 & 0.000 & 0.469 & 1.036 & 1.530 & 0.094 & 0.082 & 0.000 & 0.002 \\
\hline 41 & $\mathrm{Zr}$ & 0.000 & 0.005 & 0.000 & 1.210 & 0.125 & 0.000 & 0.015 & 0.241 & 0.000 & 0.000 \\
\hline 42 & Hf & 0.000 & 0.000 & 0.000 & 0.000 & 0.000 & 0.000 & 0.0003 & 0.000 & 0.000 & 0.000 \\
\hline 43 & $\mathrm{Sn}$ & 0.000 & 0.000 & 0.000 & 0.000 & 0.000 & 0.000 & 0.740 & 0.781 & 0.000 & 0.000 \\
\hline 44 & $\mathrm{Sb}$ & 0.000 & 0.000 & 0.000 & 0.000 & 0.000 & 0.000 & 1.075 & 0.729 & 0.000 & 0.000 \\
\hline 45 & $\mathrm{Nb}$ & 0.000 & 0.000 & 0.000 & 0.000 & 0.000 & 0.000 & 0.004 & 0.004 & 0.000 & 0.000 \\
\hline 46 & $\mathrm{U}$ & 0.000 & 0.000 & 0.000 & 0.000 & 0.000 & 0.000 & 0.001 & 0.155 & 0.000 & 0.000 \\
\hline 47 & $\mathrm{Al}$ & 0.279 & 0.961 & 0.000 & 0.000 & 0.000 & 0.167 & 0.000 & 0.000 & 0.039 & 0.000 \\
\hline 48 & $\mathrm{Fe}^{3+}$ & 0.568 & 0.882 & 1.882 & 0.000 & 0.462 & 0.047 & 0.000 & 0.000 & 0.000 & 0.000 \\
\hline 49 & $\mathrm{Sc}$ & 0.013 & 0.000 & 0.000 & 0.000 & 0.000 & 0.000 & 0.000 & 0.005 & 0.000 & 0.000 \\
\hline 50 & $\mathrm{~V}^{3+}$ & 0.012 & 0.013 & 0.000 & 0.000 & 0.038 & 0.032 & 0.000 & 0.000 & 0.000 & 0.000 \\
\hline 51 & $\mathrm{Cr}$ & 0.003 & 0.000 & 0.000 & 0.000 & 0.001 & 0.000 & 0.002 & 0.000 & 0.000 & 0.000 \\
\hline 52 & $\mathrm{Mn}^{3+}$ & 0.000 & 0.008 & 0.000 & 0.000 & 0.000 & 0.000 & 0.000 & 0.000 & 1.946 & 0.000 \\
\hline 53 & $\mathrm{Mg}$ & 0.535 & 0.021 & 0.000 & 0.321 & 0.191 & 0.244 & 0.037 & 0.002 & 0.001 & 1.675 \\
\hline 54 & $\mathrm{Fe}^{2+}$ & 0.515 & 0.000 & 0.023 & 0.000 & 0.147 & 0.000 & 0.000 & 0.000 & 0.000 & 0.001 \\
\hline 55 & $\mathrm{Mn}^{2+}$ & 0.000 & 0.000 & 0.000 & 0.000 & 0.000 & 0.000 & 0.003 & 0.000 & 0.000 & 0.286 \\
\hline 56 & $\sum Y$ site & 2.000 & 1.993 & 1.964 & 2.000 & 2.000 & 2.000 & 1.9703 & 2.000 & 1.994 & 1.964 \\
\hline
\end{tabular}


Tab. 3 Continued

\begin{tabular}{|c|c|c|c|c|c|c|c|c|c|c|c|}
\hline Row & & SG1 & SG2 & SG3 & SG4 & SG5 & SG6 & SG7 & SG8 & SG9 & SG10 \\
\hline 57 & $\mathrm{Y}+\mathrm{REE}$ & 1.214 & 0.000 & 0.000 & 0.000 & 0.000 & 0.000 & 0.000 & 0.000 & 0.000 & 0.000 \\
\hline 58 & $\mathrm{Fe}^{2+}$ & 0.376 & 0.000 & 0.000 & 0.000 & 0.012 & 0.000 & 0.000 & 0.000 & 0.000 & 0.000 \\
\hline 59 & $\mathrm{Mn}^{2+}$ & 0.065 & 0.000 & 0.000 & 0.000 & 0.034 & 0.000 & 0.000 & 0.000 & 0.000 & 0.000 \\
\hline 60 & $\mathrm{Mg}$ & 0.000 & 0.000 & 0.028 & 0.128 & 0.000 & 0.028 & 0.000 & 0.001 & 0.000 & 0.000 \\
\hline 61 & $\mathrm{Ca}$ & 1.344 & 2.997 & 2.980 & 2.937 & 2.917 & 2.980 & 3.030 & 2.999 & 3.006 & 2.125 \\
\hline 62 & $\mathrm{Na}$ & 0.000 & 0.011 & 0.000 & 0.000 & 0.037 & 0.000 & 0.000 & 0.000 & 0.000 & 0.898 \\
\hline 63 & $\sum X$ site & 3.000 & 3.008 & 3.008 & 3.065 & 3.000 & 3.008 & 3.030 & 3.000 & 3.006 & 3.023 \\
\hline 64 & $\mathrm{O}$ & 12.000 & 10.221 & 12.000 & 12.000 & 12.000 & 12.000 & 12.000 & 12.000 & 7.925 & 12.000 \\
\hline 65 & $\mathrm{OH}$ & 0.000 & 1.006 & 0.000 & 0.000 & 0.000 & 0.000 & 0.000 & 0.000 & 4.075 & 0.000 \\
\hline 66 & $\mathrm{~F}$ & 0.000 & 0.773 & 0.000 & 0.000 & 0.000 & 0.000 & 0.000 & 0.000 & 0.000 & 0.000 \\
\hline 67 & $\sum$ Anions & 12.000 & 12.000 & 12.000 & 12.000 & 12.000 & 12.000 & 12.000 & 12.000 & 12.000 & 12.000 \\
\hline 68 & Dominant Formula Type & menzerite & \multicolumn{2}{|c|}{ tetravalent $\mathrm{Z}$ garnet } & \multicolumn{3}{|c|}{ schorlomite } & \multicolumn{2}{|c|}{ bitikleite } & henritermierite & berzeliite \\
\hline 69 & Garnet Group & \multicolumn{3}{|c|}{ Garnet } & \multicolumn{3}{|c|}{ Schorlomite } & \multicolumn{2}{|c|}{ Bitikleite } & Henritermierite & Berzeliite \\
\hline 70 & Garnet Species & enzerite- & gross & dradit & imzey & chorlom & tcheon & ikleit & dzhuluite & henritermierite & berzeliite \\
\hline
\end{tabular}

Notes: The formulae were recalculated to 12 anions and 8 cations; $\mathrm{Fe}^{3+}$ and $\mathrm{Fe}^{2+}$, as well as $\mathrm{Mn}^{2+}$ and $\mathrm{Mn}^{3+}$, estimations from total $\mathrm{FeO}$ (wt. \%) contents were carried out on the basis of stoichiometric constraints using Droop's (1987) method; S1, S2, S3 from an Excel spreadsheet developed by Grew et al. (2013) to perform the calculation, cation allocation, species and group determination; S4 from Munno et al. (1980); S5 from Antao (2014); S6 from Ma et al. (2014); S7 from Galuskina et al. (2010); S8 from Galuskina et al. (2013); S9 from Antao and Cruickshank (2018); S10 from Nagashima and Armbruster (2012)

following cation exchange reaction (Eq. 1) has been calibrated by Thompson (1976) as a geothermometer (Eq. 2) based largely on comparison of natural assemblages with experimental phase equilibria:

$$
\begin{aligned}
& \mathrm{Mg}_{3} \mathrm{Al}_{2} \mathrm{Si}_{3} \mathrm{O}_{12}+\mathrm{KFe}^{2+}{ }_{3} \mathrm{AlSi}_{3} \mathrm{O}_{10}(\mathrm{OH})_{2}=\mathrm{Fe}^{2+}{ }_{3} \mathrm{Al}_{2} \mathrm{Si}_{3} \mathrm{O}_{12} \\
& \text { pyrope annite almandine } \\
& +\mathrm{KMg}_{3} \mathrm{AlSi}_{3} \mathrm{O}_{10}(\mathrm{OH})_{2} \\
& \text { phlogopite } \\
& T_{\mathrm{T} 76}^{\mathrm{Grt} B \mathrm{Bt}}\left({ }^{\circ} \mathrm{C}\right)=\frac{2739.646}{1.56-\ln K_{D}}-273.15
\end{aligned}
$$

Thompson's (1976) empirical calibration, which is essentially used for natural metapelitic assemblages of low- and medium-grade metamorphic rocks, is based on a linear relationship between $\ln K_{\mathrm{D}}$ and $1 / T$, where $K_{\mathrm{D}}=\left(\mathrm{Mg}^{\mathrm{Grt}} \times \mathrm{Fe}^{\mathrm{Bt}}\right) /\left(\mathrm{Fe}^{\mathrm{Grt}} \times \mathrm{Mg}^{\mathrm{Bt}}\right)$. An empirical calibration does not assume any pressure effect on the $\mathrm{Fe}-\mathrm{Mg}$ fractionation between garnet and biotite phases. However, by clicking the Use Input P (kbar) for Pressure-independent Garnet-biotite Geothermometer option from the Calculate menu, WinGrt uses previously entered pressure (kbar) values and estimates Thompson's (1976) calibration as a pressure-dependent geothermometer:

$T_{\mathrm{T} 76}^{\mathrm{Grt}}{ }^{\mathrm{Bt}}\left({ }^{\circ} \mathrm{C}\right)=\frac{2740+2.34 P(\mathrm{kbar})}{1.56-\ln K_{D}}-273.15$

Ferry and Spear (1978) presented experimental data at $2.07 \mathrm{kbar}$ and $550-800^{\circ} \mathrm{C}$ on the $\mathrm{Fe}^{2+}-\mathrm{Mg}$ exchange reaction between synthetic almandine-pyrope and annitephlogopite, and proposed an empirical geothermometer (Eq. 4) in systems, where $\mathrm{Fe} /(\mathrm{Fe}+\mathrm{Mg}$ ) of garnet was held at 0.9 (see row 22 in Tab. 4):

$T_{\mathrm{FS} 78}^{\mathrm{Grt}}{ }^{\mathrm{Bt}}\left({ }^{\circ} \mathrm{C}\right)=\frac{2109}{0.782-\ln K_{D}}-273.15$
Again, by clicking the Use Input P (kbar) for Pressure-independent Garnet-Biotite Geothermometer option from the Calculate menu, the program takes into account previously typed pressure (kbar) values and calculates Ferry and Spear's (1978) calibration as pressure-dependent geothermometer (Eq. 5):

$T_{\mathrm{FS} 78}^{\mathrm{Grt}-\mathrm{Bt}}\left({ }^{\circ} \mathrm{C}\right)=\frac{2089+9.56 P(\mathrm{kbar})}{0.782-\ln K_{D}}-273.15$

Hodges and Spear (1982) applied widely used geothermobarometers to pelitic schists of the Mt. Moosilauke region, New Hampshire, where the three aluminum-silicate polymorphs tightly constrain the $P-T$ conditions (see row 23 in Tab. 4). According to Hodges and Spear (1982), previous garnet-biotite calibrations (i.e. Eqs 2 and 4) may be affected by the propensity of reequilibration of garnet and biotite during cooling at geologically reasonable rates, and may underestimate the metamorphic temperatures. Hence, they presented an empirically derived more internally consistent geothermometer for a suite of garnet-biotite-plagioclasesillimanite-quartz-( \pm andalusite $)$ assemblages from Mt. Moosilauke, New Hampshire:

$T_{\mathrm{HS} 82}^{\mathrm{Grt}-\mathrm{Bt}}\left({ }^{\circ} \mathrm{C}\right)=\frac{-12454-57 P(\mathrm{kbar})-9900 X_{\mathrm{Ca}}^{\mathrm{Grt}}}{3 \times 1.9872 \ln K_{D}-4.5 X_{\mathrm{Ca}}^{\mathrm{Grt}}}-273.15(6)$

where $X_{\mathrm{Ca}}^{\mathrm{Gr}}=\frac{\mathrm{Ca}}{\mathrm{Ca}+\mathrm{Fe}^{2+}+\mathrm{Mg}+\mathrm{Mn}}$.

In estimating the temperature conditions of biotitegarnet equilibria in metamorphic rocks, Perchuk and Lavrent'eva (1983) proposed a geothermometer using predominantly natural minerals as reactants for their experiments at $550-1000^{\circ} \mathrm{C}$ and $5-7 \mathrm{kbar}$ : 


\begin{tabular}{|c|c|c|c|c|c|c|c|c|c|c|c|c|c|c|c|c|c|c|c|c|c|c|c|c|c|c|c|c|c|c|c|c|}
\hline \multicolumn{27}{|c|}{ A Windows Program for Garnet Supergroup Minerals } & \multicolumn{6}{|c|}{ Calculation Screen } \\
\hline & 38 & 39 & 40 & 41 & 42 & 43 & 44 & 45 & 46 & 47 & 48 & 49 & 50 & 51 & 52 & 53 & 54 & 55 & 56 & 57 & 58 & 59 & 60 & 61 & 62 & 63 & 64 & 65 & 66 & 67 & 68 & \\
\hline Sample & {$[\mathrm{Si}$} & $\pi$ & $\mathrm{Zr}$ & $\mathrm{Hf}$ & Th & Sn & Al & $\mathrm{Cr}$ & $V+3$ & $\mathrm{Fe} 3+$ & $\mathrm{Mn} 3+$ & Sc & $+R E E$ & $\mathrm{Fe} 2+$ & $\mathrm{Mg}$ & $M n$ & $2 n$ & $\mathrm{Ca}$ & $\mathrm{Na}$ & نا & $P$ & V5+ & $\mathrm{Sb}$ & As & $\mathrm{Nb}$ & U & $\mathrm{Te}$ & $\mathrm{F}$ & $\mathrm{O}=\mathrm{F}$ & $\mathrm{H} 4$ & Total](apfu) & \\
\hline Menzer & 2.762 & 0.075 & 0.000 & 0.000 & 0.000 & 0.000 & 0.517 & 0.003 & 0.012 & 0.568 & 0.000 & 0.013 & 1.214 & 0.891 & 0.535 & 0.065 & 0.0001 & $1.344 \mathrm{C}$ & 0.000 & 0.0000 & 0.0000 & 0.000 & 0.000 & 0.0000 & 0.000 & 0.000 & 0.000 & 0.000 & 0.000 & 0.000 & 00 & \\
\hline Pyrope & 94 & 0.049 & 0.000 & 0.000 & 0.000 & 0.000 & 1.832 & 0.011 & 0.000 & 0.080 & 0.000 & 0.000 & 0.000 & 0.537 & 2.125 & 0.018 & 0.0000 & $0.345 \mathrm{C}$ & 0.008 & 0.0000 & 0.0000 & 0.000 & 0.000 & 0.0000 & 0.000 & 0.000 & 0.000 & 0.000 & 0.000 & 0.000 & 000 & \\
\hline ossular & 931 & 0.023 & 0.000 & 0.000 & 0.000 & 0.000 & 1.937 & 0.000 & 0.000 & 0.089 & 0.040 & 0.000 & 0.000 & 0.000 & 0.000 & 0.005 & 0.0002 & 2.9620 & 0.000 & 0.0000 & 0.0000 & 0.000 & 0.000 & 0.0000 & 0.000 & 0.000 & 0.000 & 0.000 & 0.000 & 0.013 & 000 & \\
\hline ne & 876 & 0.000 & 0.000 & 0.000 & 0.000 & 0.000 & 2.063 & 0.000 & 0.000 & 0.000 & 0.000 & 0.000 & 0.000 & 0.000 & 0.000 & $2.831 \mathrm{C}$ & 0.0000 & 0.0890 & 0.000 & 0.0000 & 0.0000 & 0.000 & 0.000 & 0.0000 & 0.000 & 0.000 & 0.000 & 0.000 & 0.000 & 0.117 & 976 & \\
\hline nandine & 2.955 & 0.075 & 0.000 & 0.000 & 0.000 & 0.000 & 2.016 & 0.000 & 0.000 & 0.000 & 0.000 & 0.000 & 0.000 & 2.519 & 0.162 & 0.173 & 0.0000 & 0.050 & 0.008 & 0.0000 & 0.0030 & 0.000 & 0.000 & 0.0000 & 0.000 & 0.000 & 0.000 & 0.000 & 0.000 & 0.000 & 962 & \\
\hline Goldmante & 2.868 & 0.031 & 0.000 & 0.000 & 0.000 & 0.000 & 0.185 & 0.002 & 1.821 & 0.051 & 0.012 & 0.000 & 0.000 & 0.000 & 0.000 & 0.000 & 0.0003 & 3.0960 & 0.000 & 0.0000 & 0.0000 & 0.000 & 0.000 & 0.0000 & 0.000 & 0.000 & 0.000 & 0.000 & 0.000 & 0.000 & 066 & \\
\hline Momoite & 3.035 & 0.009 & 0.000 & 0.000 & 0.000 & 0.000 & 0.595 & 0.019 & 1.331 & 0.000 & 0.000 & 0.000 & 0.000 & 0.025 & 0.010 & 1.692 & 0.0001 & $1.267 \mathrm{C}$ & 0.000 & 0.0000 & 0.0000 & 0.000 & 0.000 & 0.0000 & 0.000 & 0.000 & 0.000 & 0.000 & 0.000 & 0.000 & 983 & \\
\hline Knorringte & 3.007 & 0.006 & 0.000 & 0.000 & 0.000 & 0.000 & 0.865 & $1.041 \mathrm{C}$ & $0.000 \mathrm{C}$ & 0.068 & 0.000 & 0.000 & 0.000 & 0.411 & 1.906 & 0.038 & 0.0000 & 0.657 & 0.000 & 0.0000 & 0.0000 & 0.000 & 0.000 & 0.0000 & 0.000 & 0.000 & 0.000 & 0.000 & 0.000 & 0.000 & 000 & \\
\hline varovite & 3.028 & 0.000 & 0.000 & 0.000 & 0.000 & 0.000 & 0.187 & 1.792 & 0.000 & 0.000 & 0.000 & 0.000 & 0.000 & 0.025 & 0.061 & 0.000 & 0.0002 & $2.887 \mathrm{C}$ & 0.000 & 0.0000 & 0.090 & 0.000 & 0.000 & 0.0000 & 0.000 & 0.000 & 0.000 & 0.000 & 0.000 & 0.000 & 902 & \\
\hline Andradtie & 3.059 & 0.000 & 0.000 & 0.000 & 0.000 & 0.000 & 0.000 & 0.000 & 0.000 & 1.882 & 0.000 & 0.000 & 0.000 & 0.023 & 0.000 & 0.000 & 0.0003 & $3.036 \mathrm{C}$ & 0.000 & 0.0000 & $0.000^{\circ} \mathrm{C}$ & 0.000 & 0.000 & 0.0000 & 0.000 & 0.000 & 0.000 & 0.000 & 0.000 & 0.000 & .000 & \\
\hline Calderte & 3.004 & 0.010 & 0.000 & 0.000 & 0.000 & 0.000 & 0.582 & 0.000 & 0.000 & 1.398 & 0.000 & 0.000 & 0.000 & 0.181 & 0.006 & $1.761 \mathrm{C}$ & 0.0001 & 1.0550 & 0.000 & 0.0000 & 0.0000 & 0.000 & 0.000 & 0.0000 & 0.000 & 0.000 & 0.000 & 0.000 & 0.000 & 0.000 & 97 & \\
\hline Majortie & 3.728 & 0.000 & 0.000 & 0.000 & 0.000 & 0.000 & 0.699 & 0.000 & $0.000 \mathrm{C}$ & 0.000 & 0.000 & 0.000 & 0.000 & 0.000 & 3.496 & 0.000 & 0.0000 & $0.000 \mathrm{C}$ & 0.000 & 0.0000 & 0.0000 & 0.000 & 0.000 & 0.0000 & 0.000 & 0.000 & 0.000 & 0.000 & 0.000 & 0.000 & 922 & \\
\hline Morimotote & 2.319 & 1.199 & 0.062 & 0.000 & 0.000 & 0.000 & 0.098 & 0.000 & 0.000 & 0.740 & 0.000 & 0.000 & 0.000 & 0.560 & 0.112 & 0.017 & 0.0002 & 2.8930 & 0.000 & 0.0000 & 0.0000 & 0.000 & 0.000 & 0.0000 & 0.000 & 0.000 & 0.000 & 0.000 & 0.000 & 0.000 & 000 & \\
\hline Kimzeyte & 1.507 & 0.474 & 1.210 & 0.000 & 0.000 & 0.000 & 1.001 & 0.000 & 0.000 & 0.487 & 0.000 & 0.000 & 0.000 & 0.000 & 0.449 & 0.000 & 0.0002 & $2.937 \mathrm{C}$ & $0.000 \mathrm{C}$ & 0.0000 & 0.0000 & 0.000 & 0.000 & 0.0000 & 0.000 & 0.000 & 0.000 & 0.000 & 0.000 & 0.000 & 065 & \\
\hline Schoriomte & 2.214 & 1.036 & 0.125 & 0.000 & 0.000 & 0.000 & 0.073 & 0.001 & 0.038 & 1.175 & 0.000 & 0.000 & 0.000 & 0.159 & 0.191 & 0.034 & 0.0002 & $2.917 \mathrm{C}$ & 0.037 & 0.0000 & 0.0000 & 0.000 & 0.000 & 0.0000 & 0.000 & 0.000 & 0.000 & 0.000 & 0.000 & 0.000 & 000 & \\
\hline erimaste & 1.656 & 0.595 & 0.811 & 0.017 & 0.000 & 0.010 & 0.465 & 0.000 & 0.000 & 1.303 & 0.000 & 0.019 & 0.002 & 0.056 & 0.033 & 0.000 & 0.0003 & $3.021 \mathrm{C}$ & 0.000 & 0.0000 & 0.0020 & 0.000 & 0.000 & 0.0000 & 0.008 & 0.000 & 0.000 & 0.000 & 0.000 & 0.000 & 000 & \\
\hline turite & 0.552 & 0.359 & 0.013 & 0.000 & 0.000 & 1.462 & 0.609 & 0.001 & 0.000 & 1.633 & 0.000 & 0.000 & 0.000 & 0.050 & 0.003 & 0.000 & 0.0002 & $2.989 \mathrm{C}$ & 0.000 & 0.0000 & 0.0000 & 0.001 & 0.325 & 0.0000 & 0.002 & 0.000 & 0.000 & 0.000 & 0.000 & 0.000 & 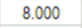 & \\
\hline Hutcheonite & 1.680 & 1.530 & 0.000 & 0.000 & 0.000 & 0.000 & 1.487 & 0.000 & 0.032 & 0.047 & 0.000 & 0.000 & 0.000 & 0.000 & 0.252 & 0.000 & 0.0002 & $2.980 \mathrm{C}$ & 0.000 & 0.0000 & 0.0000 & 0.000 & 0.000 & 0.0000 & 0.000 & 0.000 & 0.000 & 0.000 & 0.000 & 0.000 & 007 & \\
\hline Bokle & 0.031 & 0.223 & 0.015 & 0.000 & 0.000 & 0.740 & 1.530 & 0.002 & 0.000 & 1.206 & 0.000 & 0.000 & 0.000 & 0.103 & 0.037 & 0.003 & 0.0003 & $3.030 \mathrm{C}$ & 0.000 & 0.0000 & 0.0000 & 0.001 & 1.075 & 0.0000 & 0.004 & 0.001 & 0.000 & 0.000 & 0.000 & 0.000 & .000 & \\
\hline chulute & 0.033 & 0.231 & 0.241 & 0.000 & 0.000 & 0.781 & 0.853 & 0.000 & 0.000 & 1.750 & 0.000 & 0.005 & 0.000 & 0.216 & 0.003 & 0.000 & 0.0002 & $2.999 \mathrm{C}$ & 0.000 & 0.0000 & 0.0000 & 0.000 & 0.729 & 0.0000 & 0.004 & 0.155 & 0.000 & 0.000 & 0.000 & 0.000 & 0 & \\
\hline Elbru & 0.098 & 0.199 & 40 & 0.009 & 0.018 & 0.255 & 0.539 & 0.000 & 0.000 & 1.576 & 0.000 & 0.000 & 0.001 & 0.559 & 0.004 & 0.000 & 0.0003 & 3.040 & 0.000 & 0.0000 & 0.0000 & 0.004 & 0.000 & 0.0000 & 0.000 & 0.658 & 0.000 & 0.000 & 0.000 & 0.000 & 000 & \\
\hline Hotst: & 1.862 & 0.000 & 0.000 & 0.000 & 0.000 & 0.000 & 1.293 & 0.000 & 0.000 & 0.000 & 0.646 & 0.000 & 0.000 & 0.000 & 0.000 & 0.000 & 0.0003 & $3.027 \mathrm{C}$ & $0.000 \mathrm{C}$ & 0.0000 & 0.0000 & 0.000 & 0.000 & 0.0000 & 0.000 & 0.000 & 0.000 & 0.000 & 0.000 & 1.170 & 998 & \\
\hline & 1.988 & 0.000 & 0.000 & 0.000 & 0.000 & 0.000 & 0.039 & 0.000 & $0.000 \mathrm{C}$ & 0.000 & 1.946 & 0.000 & 0.000 & 0.000 & 0.001 & 0.000 & 0.0003 & 3.0060 & 0.000 & 0.0000 & 0.0000 & 0.000 & 0.000 & 0.0000 & 0.000 & 0.000 & 0.000 & 0.000 & 0.000 & 1.019 & .000 & \\
\hline Palenzonate & 0.183 & 0.003 & 0.000 & 0.000 & 0.000 & 0.000 & 0.002 & 0.000 & $0.000 \mathrm{c}$ & 0.000 & 0.000 & 0.000 & 0.000 & 0.003 & 0.003 & $1.818 \mathrm{C}=$ & 0.0002 & $2.219 \mathrm{C}$ & $0.771 \mathrm{C}$ & 0.0000 & 0.0002 & 2.712 & 0.000 & 0.1660 & 0.000 & 0.000 & 0.000 & 0.000 & 0.000 & 0.000 & 7.881 & \\
\hline 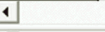 & & & & & & & & & & & & & & & & & & & & & & & & & & & & & & & & \\
\hline & & & & & & & & & & & & & & & & & & & & & & & & & & & & & & & & \\
\hline
\end{tabular}

A Windows Program for Garnet Supergroup Minerals

Calculation Screen

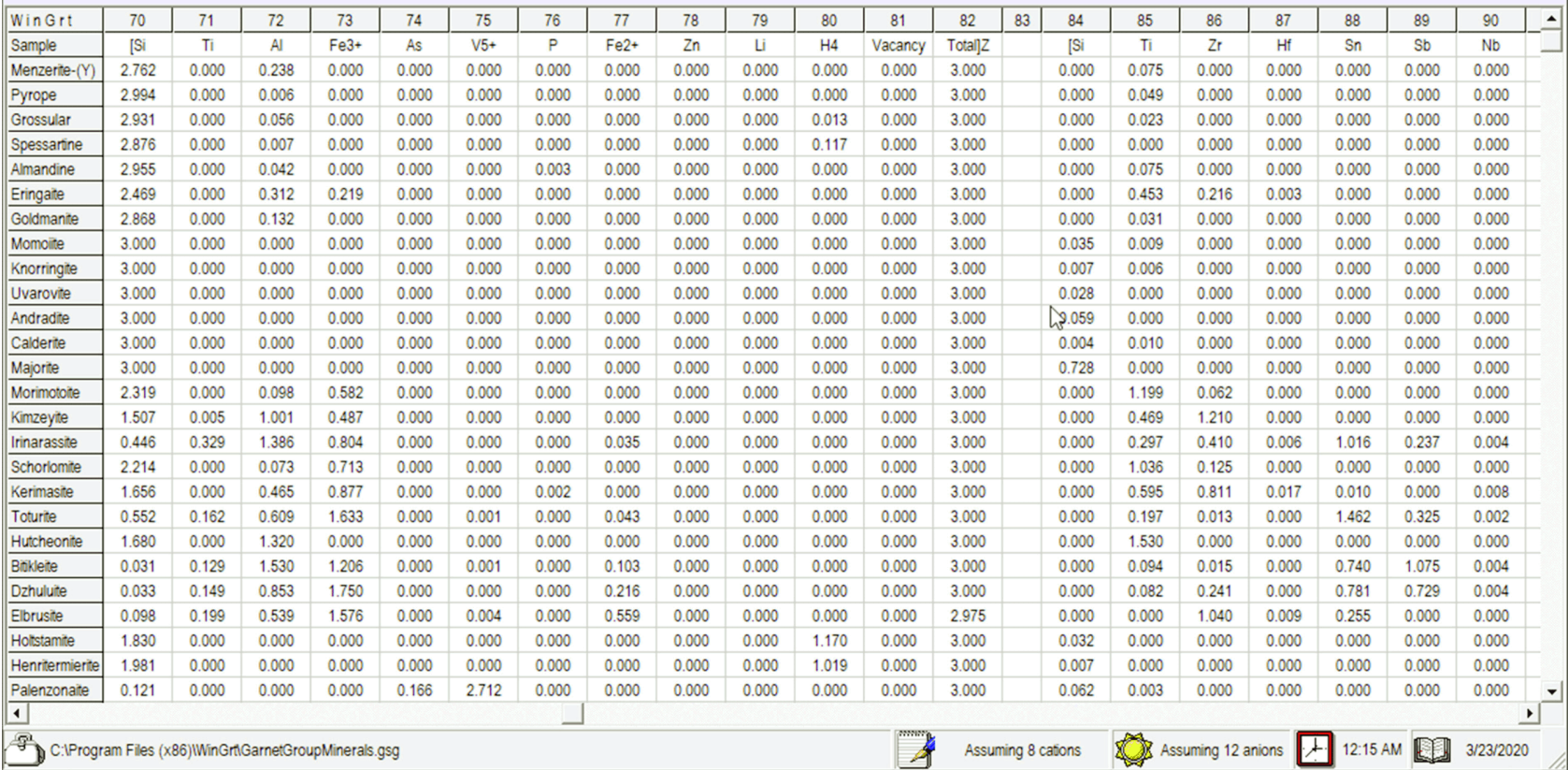

Fig. 2 Screenshots of the WinGrt Calculation Screen window. a - Recalculated cations of garnet supergroup mineral analyses (apfu). b - Cations allocation at the $Z, Y$, and $X$ sites $(a p f u)$. 
WinGrt

Edit Excel Graph About Help

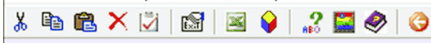

A Windows Program for Garnet Supergroup Minerals

Calculation Screen

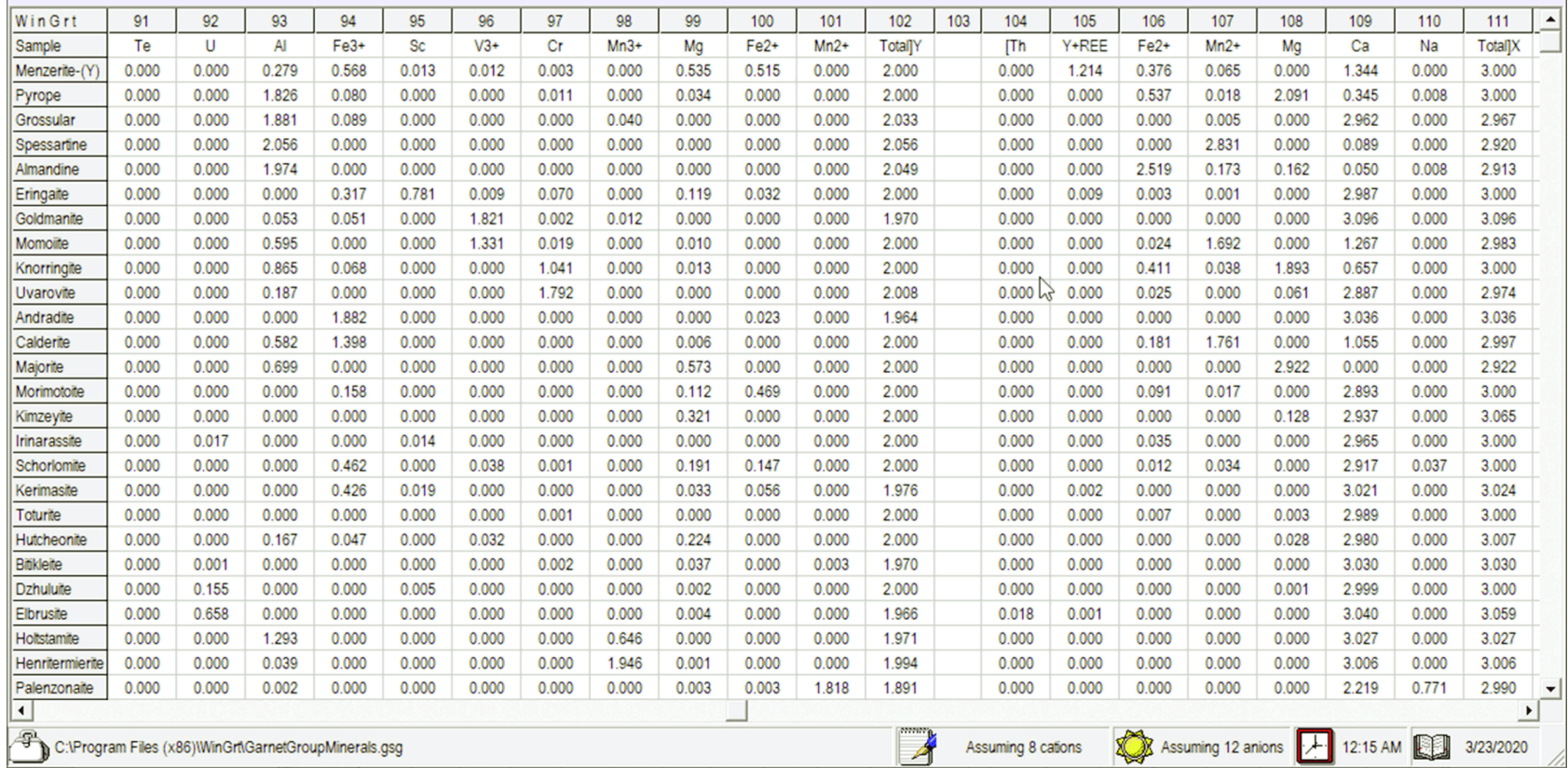

Wingrt

Edit Excel Graph About Help

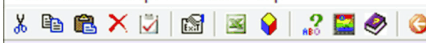

d

A Windows Program for Garnet Supergroup Minerals

Calculation Screen

\begin{tabular}{|c|c|c|c|c|c|c|c|c|c|c|c|c|c|c|c|c|c|}
\hline Wingrt & 118 & 119 & 120 & 121 & 122 & 123 & 124 & 125 & 126 & 127 & 128 & 129 & 130 & 131 & 132 & 133 & $\Delta$ \\
\hline Sample & Monovalent Z & Divalent Z & Trivalent Z & Tetravalent $Z$ & Pentavalent $Z$ & Hydrogen for $Z$ & Vacancy at $z$ & Divalent $Y$ & Trivalent $Y$ & Tetravalent $Y$ & Pentavalent $Y$ & Hexavalent $Y$ & Monovalent X & Divalent $X$ & Trivalent $\mathrm{X}$ & Tetravalent X & t \\
\hline Menzerte- $(\mathrm{Y})$ & 0.00 & 0.00 & 0.24 & 2.76 & 0.00 & 0.00 & 0.00 & 1.05 & 0.88 & 0.07 & 0.00 & 0.00 & 0.00 & 1.79 & 1.21 & 0.00 & \\
\hline Pyrope & 0.00 & 0.00 & 0.01 & 2.99 & 0.00 & 0.00 & 0.00 & 0.03 & 1.92 & 0.05 & 0.00 & 0.00 & 0.01 & 2.99 & 0.00 & 0.00 & \\
\hline Grossular & 0.00 & 0.00 & 0.06 & 2.93 & 0.00 & 0.01 & 0.00 & 0.00 & 2.01 & 0.02 & 0.00 & 0.00 & 0.00 & 2.97 & 0.00 & 0.00 & \\
\hline Spessartine & 0.00 & 0.00 & 0.01 & 2.88 & 0.00 & 0.12 & 0.00 & 0.00 & 2.06 & 0.00 & 0.00 & 0.00 & 0.00 & 2.92 & 0.00 & 0.00 & \\
\hline Almandine & 0.00 & 0.00 & 0.04 & 2.96 & 0.00 & 0.00 & 0.00 & 0.00 & 1.97 & 0.07 & 0.00 & 0.00 & 0.01 & 2.91 & 0.00 & 0.00 & \\
\hline Eringare & 0.00 & 0.00 & 0.53 & 2.47 & 0.00 & 0.00 & 0.00 & 0.15 & 1.18 & 0.67 & 0.00 & 0.00 & 0.00 & 2.99 & 0.01 & 0.00 & \\
\hline Goldmante & 0.00 & 0.00 & 0.13 & 2.87 & 0.00 & 0.00 & 0.00 & 0.00 & 1.94 & 0.03 & 0.00 & 0.00 & 0.00 & 3.10 & 0.00 & 0.00 & \\
\hline Momoite & 0.00 & 0.00 & 0.00 & 3.00 & 0.00 & 0.00 & 0.00 & 0.01 & 1.95 & 0.04 & 0.00 & 0.00 & 0.00 & 2.98 & 0.00 & 0.00 & \\
\hline Uvarovte & 0.00 & 0.00 & 0.00 & 3.00 & 0.00 & 0.00 & 0.00 & 0.00 & 1.98 & 0.03 & 0.00 & 0.00 & 0.00 & 2.97 & 0.00 & 0.00 & \\
\hline Andradte & 0.00 & 0.00 & 0.00 & 3.00 & 0.00 & 0.00 & 0.00 & 0.02 & 1.88 & 0.06 & $0.9 \rho$ & 0.00 & 0.00 & 3.04 & 0.00 & 0.00 & \\
\hline Calderte & 0.00 & 0.00 & 0.00 & 3.00 & 0.00 & 0.00 & 0.00 & 0.01 & 1.98 & 0.01 & $0 . d a^{5}$ & 0.00 & 0.00 & 3.00 & 0.00 & 0.00 & \\
\hline Majorte & 0.00 & 0.00 & 0.00 & 3.00 & 0.00 & 0.00 & 0.00 & 0.57 & 0.70 & 0.73 & 0.00 & 0.00 & 0.00 & 2.92 & 0.00 & 0.00 & \\
\hline Morimotote & 0.00 & 0.00 & 0.68 & 2.32 & 0.00 & 0.00 & 0.00 & 0.58 & 0.16 & 1.26 & 0.00 & 0.00 & 0.00 & 3.00 & 0.00 & 0.00 & \\
\hline Kimzeyte & 0.00 & 0.00 & 1.49 & 1.51 & 0.00 & 0.00 & 0.00 & 0.32 & 0.00 & 1.68 & 0.00 & 0.00 & 0.00 & 3.06 & 0.00 & 0.00 & \\
\hline Irinarasste & 0.00 & 0.04 & 2.19 & 0.77 & 0.00 & 0.00 & 0.00 & 0.00 & 0.01 & 1.73 & 0.24 & 0.02 & 0.00 & 3.00 & 0.00 & 0.00 & \\
\hline Schorlomite & 0.00 & 0.00 & 0.79 & 2.21 & 0.00 & 0.00 & 0.00 & 0.34 & 0.50 & 1.16 & 0.00 & 0.00 & 0.04 & 2.96 & 0.00 & 0.00 & \\
\hline Kerimaste & 0.00 & 0.00 & 1.34 & 1.66 & 0.00 & 0.00 & 0.00 & 0.09 & 0.45 & 1.43 & 0.01 & 0.00 & 0.00 & 3.02 & 0.00 & 0.00 & \\
\hline Boklete & 0.00 & 0.10 & 2.74 & 0.16 & 0.00 & 0.00 & 0.00 & 0.04 & 0.00 & 0.85 & 1.08 & 0.00 & 0.00 & 3.03 & 0.00 & 0.00 & \\
\hline Dzhulute & 0.00 & 0.22 & 2.60 & 0.18 & 0.00 & 0.00 & 0.00 & 0.00 & 0.01 & 1.10 & 0.73 & 0.16 & 0.00 & 3.00 & 0.00 & 0.00 & \\
\hline Elbruste & 0.00 & 0.56 & 2.11 & 0.30 & 0.00 & 0.00 & 0.00 & 0.00 & 0.00 & 1.30 & 0.00 & 0.66 & 0.00 & 3.04 & 0.00 & 0.02 & \\
\hline Hotstamte & 0.00 & 0.00 & 0.00 & 1.83 & 0.00 & 1.17 & 0.00 & 0.00 & 1.94 & 0.03 & 0.00 & 0.00 & 0.00 & 3.03 & 0.00 & 0.00 & \\
\hline Henritermiertie & 0.00 & 0.00 & 0.00 & 1.98 & 0.00 & 1.02 & 0.00 & 0.00 & 1.99 & 0.01 & 0.00 & 0.00 & 0.00 & 3.01 & 0.00 & 0.00 & \\
\hline Palenzonate & 0.00 & 0.00 & 0.00 & 0.12 & 2.88 & 0.00 & 0.00 & 1.82 & 0.00 & 0.06 & 0.00 & 0.00 & 0.77 & 2.22 & 0.00 & 0.00 & - \\
\hline \multicolumn{17}{|l|}{4} & \\
\hline${ }^{2}$ C.Pro & 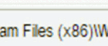 & & 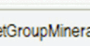 & & & & & & & 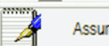 & ming 8 casons & & & $1+$ & $\mathbb{t}$ & $3 / 23 / 2020$ & \\
\hline
\end{tabular}

Fig. 2 Continued. c - Cations allocation at the $Z, Y$, and $X$ sites (apfu). d - Sums of homovalent cations by site (apfu). 


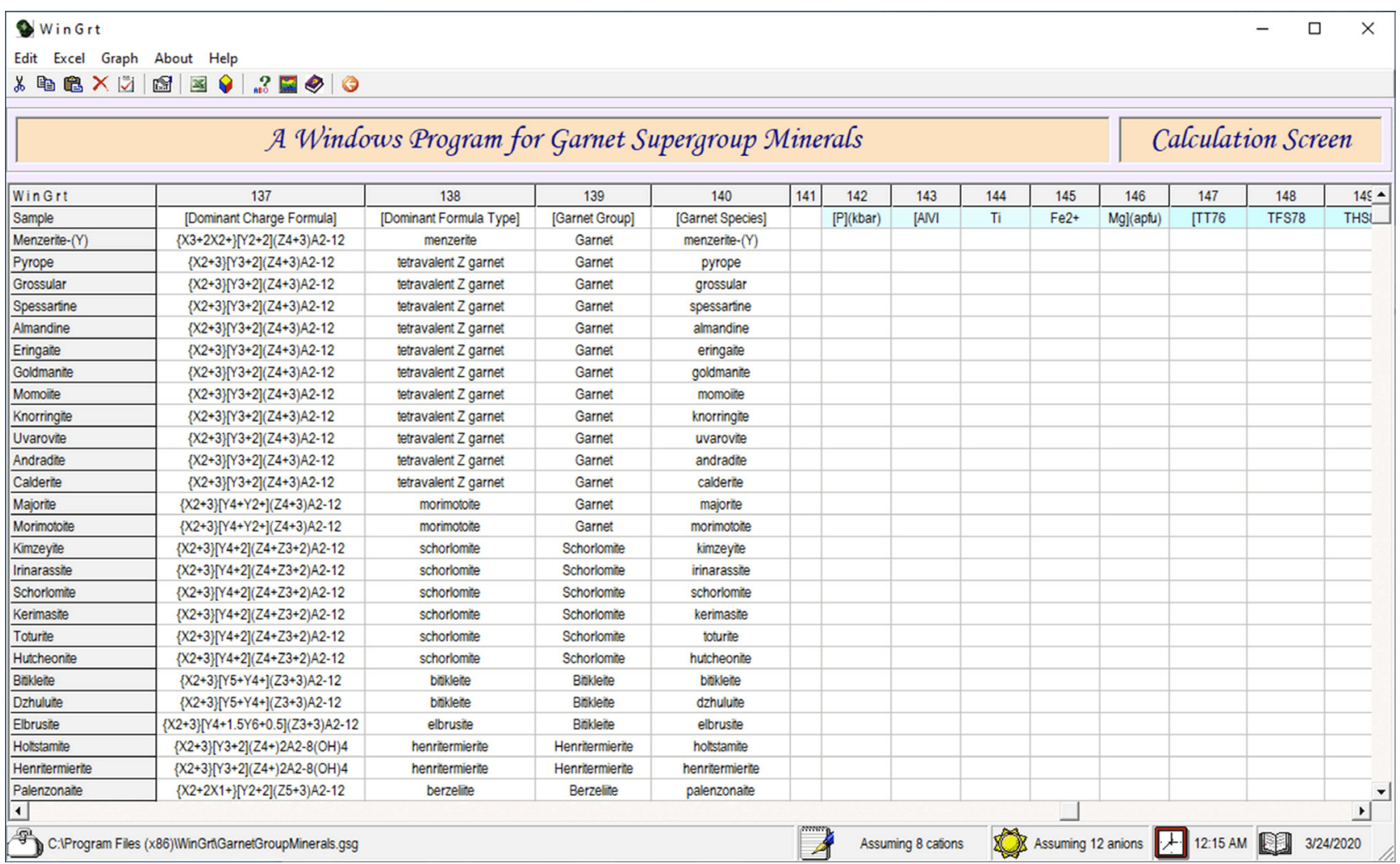

Fig. 3 Screenshots of the WinGrt Calculation Screen window showing the dominant charge formula, formula type, garnet group and garnet species.

$T_{\mathrm{PL} 83}^{\mathrm{Grt}-\mathrm{Bt}}\left({ }^{\circ} \mathrm{C}\right)=\frac{7843.7-0.0246 P(\mathrm{kbar})-6}{1.987 \ln K_{D}+5.699}-273.15$

Taking into account the negative Clausius-Clapeyron slopes, Thoenen (1989) suggested a corrected version of garnet-biotite geothermometer developed by Perchuk and Lavrent'eva (1983):

$T_{\mathrm{PL} 83}^{\mathrm{Grt}-\mathrm{Bt}}\left({ }^{\circ} \mathrm{C}\right)=\frac{3890+9.56 P(\mathrm{kbar})}{2.868-\ln K_{D}}-273.15$

WinGrt uses this revised version of equation by Thoenen (1989) in estimation of the Perchuk and Lavrent'eva (1983) calibration (see row 24 in Tab. 4).

Considering a reliable temperature estimate for a wide compositional range of natural garnet and biotite assemblages, Ganguly and Saxena (1984) extensively studied the mixing behavior of non-ideality in quaternary $(\mathrm{Fe}-\mathrm{Mg}-\mathrm{Ca}-$ $\mathrm{Mn}$ ) garnet solid solution and an ideal mixing of $\mathrm{Fe}-\mathrm{Mg}$ in biotite. Thus, on the basis of thermodynamic and statistical treatment of natural data, they formulated the following garnet-biotite geothermometer (see row 25 in Tab. 4):

$T_{\mathrm{GS} 84}^{\mathrm{Grt}-\mathrm{Bt}}\left({ }^{\circ} \mathrm{C}\right)=\frac{-17371-79.5 P(\mathrm{kbar})+0.8 \times 9497.7}{8.3144 \ln K_{D}-0.782}$

$\frac{-W_{\mathrm{MgFe}}^{\mathrm{Grt}}\left(X_{\mathrm{Fe}}^{\mathrm{Grt}}-X_{\mathrm{Mg}}^{\mathrm{Grt}}\right)-12552 X_{\mathrm{Ca}}^{\mathrm{Grt}}-12552 X_{\mathrm{Mn}}^{\mathrm{Grt}}}{8.3144 \ln K_{D}-0.782}-273.15(9)$ where $W_{\mathrm{MgFe}}^{\mathrm{Grt}}=\frac{837 X_{\mathrm{Mg}}^{\mathrm{Grt}}+10460 X_{\mathrm{Fe}}^{\mathrm{Grt}}}{X_{\mathrm{Mg}}^{\mathrm{Grt}}+X_{\mathrm{Fe}}^{\mathrm{Grt}}}$ and $X_{i}^{\mathrm{Grt}}=\frac{i}{\mathrm{Mg}+\mathrm{Fe}^{2+}+\mathrm{Mn}+\mathrm{Ca}}$.

Since the first attempts of empirical calibration of distribution coefficient $\left(K_{\mathrm{D}}\right)$ as a function of temperature, biotite-garnet geothermometers (e.g. Thompson 1976; Ferry and Spear 1978) have been widely used in estimation of temperature conditions in medium- to high-grade pelitic and semipelitic rocks. However, application of these empirical calibrations to high-grade metamorphic terranes, such as granulite-facies rocks, showed internally inconsistent and highly variable results (Bohlen and Essene 1980). Indares and Martignole (1985) reevaluated the various garnet-biotite geothermometers in granulitefacies rocks and, based on their own observations and analyses, provided two improved calibrations (Eqs 10 and 11) of Ferry and Spear's (1978) garnet-biotite geothermometer (see rows 26-27 in Tab. 4) that take into account the effects of $\mathrm{Ti}$ and $\mathrm{Al}$ in the biotite solid-solution:

$$
\begin{aligned}
& T 1_{\mathrm{IM} 85}^{\mathrm{Grt}-\mathrm{Bt}}\left({ }^{\circ} \mathrm{C}\right)=\frac{12454+57 P(\mathrm{kbar})-3 \times 1590\left(\mathrm{Al}_{\mathrm{Bt}}^{\mathrm{VI}} / 3\right)}{4.662-5.9616 \ln K_{D}} \\
& \frac{+3 \times 7451\left(\mathrm{Ti}_{\mathrm{Bt}} / 3\right)+9000\left(X_{\mathrm{Ca}}^{\mathrm{Grt}}+X_{\mathrm{Mn}}^{\mathrm{Grt}}\right)}{4.662-5.9616 \ln K_{D}}-273.15
\end{aligned}
$$


Wingrt

Edit Excel Graph About Help

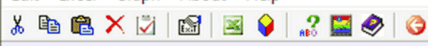

A Windows Program for Garnet Supergroup Minerals

Calculation Screen

\begin{tabular}{|c|c|c|c|c|c|c|c|c|c|c|c|c|c|c|c|}
\hline Wing it & 142 & 143 & 144 & 145 & 146 & 147 & 148 & 149 & 150 & 151 & 152 & 153 & 154 & 155 & 156 \\
\hline Sample & [P](Kbar) & {$[A|N|$} & Ti & $\mathrm{Fe} 2+$ & Mg](aptu) & [TT76 & TFS78 & THS82 & TPL83 & TGS84 & T11M85 & T21M85 & TD91 & TDB92HW84 & TDB92GS84](OC) \\
\hline LT122 & 5 & 0.459 & 0.0455 & 1.191 & 1.219 & 615 & 641 & 659 & 623 & 553 & 588 & 620 & 664 & 577 & 633 \\
\hline PR54 & 5 & 0.4045 & 0.0955 & 1.1125 & 1.3405 & 531 & 529 & 547 & 562 & 478 & 469 & 493 & 566 & 551 & 568 \\
\hline PR40 & 5 & 0.45 & 0.082 & 1.22 & 1.126 & 554 & 559 & 579 & 579 & 532 & 500 & 527 & 591 & 454 & 450 \\
\hline P542 & 5 & 0.3605 & 0.1105 & 1.142 & 1.2955 & 578 & 591 & 610 & 597 & 517 & 526 & 546 & 624 & 584 & 633 \\
\hline PR33 & 5 & 0.3155 & 0.101 & 1.1615 & 1.3075 & 506 & 497 & 540 & 543 & 491 & 486 & 486 & 574 & 461 & 456 \\
\hline SW163 & 5 & 0.4 & 0.074 & 0.895 & 1.54 & 491 & 478 & 532 & 532 & 439 & 492 & 487 & 569 & 653 & 719 \\
\hline SW198 & 5 & 0.3225 & 0.104 & 0.7745 & 1.741 & 429 & 402 & 451 & 483 & 368 & 401 & 401 & 494 & 707 & 769 \\
\hline $\mathrm{T} 172$ & 5 & 0.523 & 0.0605 & 0.884 & 1.4435 & 493 & 481 & 541 & 533 & 451 & 495 & 496 & 571 & 612 & 663 \\
\hline 01 & 5 & 0.3705 & 0.098 & 1.105 & 1.3345 & 510 & 502 & 580 & 546 & 513 & 543 & 522 & 621 & 464 & 475 \\
\hline PF3 & 5 & 0.353 & 0.083 & 0.9655 & 1.501 & 490 & 477 & 551 & 531 & 463 & 513 & 501 & 594 & 556 & 587 \\
\hline SW158 & 5 & 0.537 & 0.0515 & 0.733 & 1.589 & 394 & 361 & 437 & 454 & 375 & 404 & 399 & 476 & 551 & 542 \\
\hline SW151 & 5 & 0.4225 & 0.093 & 0.899 & 1.502 & 460 & 440 & 535 & 507 & 455 & 502 & 479 & 580 & 515 & 530 \\
\hline \multicolumn{16}{|l|}{4} \\
\hline \multicolumn{9}{|c|}{ C.IProgram Files (x86)WinGriGrBioSamples.gsg } & H & \multicolumn{4}{|c|}{ Assuming 8 casons } & \multicolumn{2}{|c|}{ 12:15 AM [3/24/2020 } \\
\hline
\end{tabular}

WinGrt

Edit Excel Graph About Help

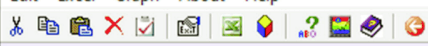

A Windows Program for Garnet Supergroup Minerals

Calculation Screen

\begin{tabular}{|c|c|c|c|c|c|c|c|c|c|c|c|c|c|c|c|}
\hline Wingrt & 158 & 159 & 160 & 161 & 162 & 163 & 164 & 165 & 166 & 167 & 168 & 169 & 170 & 171 & 172 \\
\hline Sample & {$[P]($ kbar) } & {$[A]\left(t^{\circ}\right)$} & $\pi$ & $\mathrm{Fe}\left(\mathrm{O}^{\circ}\right)$ & Mg](aptu) & [TRG74 & TEG79 & TG79 & TP85 & TK88 & TA94 & TG96 & TL98 & TKROO & TN09]0C)Gr-CpX \\
\hline grt1 & 25 & 0.437 & 0.191 & 0.191 & 0.536 & 1012 & 1037 & 1072 & 1025 & 1026 & 1007 & 1077 & 1003 & 1087 & 1055 \\
\hline$g+2$ & 25 & 0.468 & 0.221 & 0.221 & 0.507 & 1071 & 1129 & 1142 & 1122 & 1146 & 1137 & 1144 & 1123 & 1183 & 1126 \\
\hline grn3 & 25 & 0.465 & 0.228 & 0.228 & 0.538 & 1104 & 1157 & 1171 & 1152 & 1178 & 1170 & 1174 & 1152 & 1221 & 1165 \\
\hline gra & 25 & 0.376 & 0.216 & 0.216 & 0.593 & 1050 & 1085 & 1112 & 1076 & 1086 & 1067 & 1118 & 1060 & 1140 & 1101 \\
\hline gr5 & 25 & 0.388 & 0.202 & 0.202 & 0.664 & 1112 & 1149 & 1172 & 1143 & 1160 & 1129 & 1182 & 1115 & 1218 & 1175 \\
\hline \multicolumn{14}{|l|}{4} & \multicolumn{2}{|l|}{$\square$} \\
\hline \multicolumn{9}{|c|}{ C.IProgram Files (x86)WinGriGrCpxSamples.gsg } & 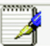 & \multicolumn{4}{|c|}{ Assuming 8 catons } & \multicolumn{2}{|c|}{ 12:15 AM $3 / 24 / 2020$} \\
\hline
\end{tabular}

WinGrt

Edit Excel Graph About Help

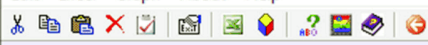

A Windows Program for Garnet Supergroup Minerals

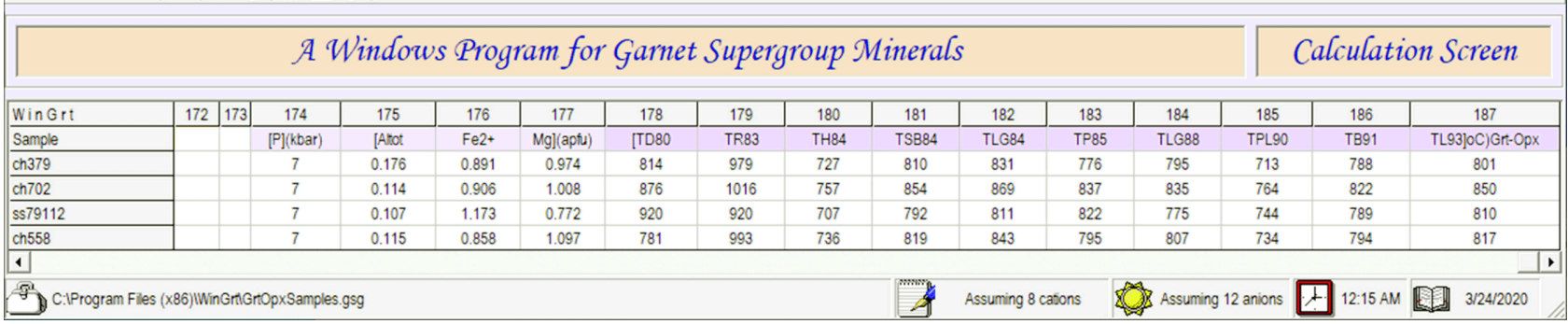

Fig. 4 Screenshots of the WinGrt Calculation Screen window. a - Input $P$ (kbar) and $\mathrm{Al}^{\mathrm{VI}}$, $\mathrm{Ti}_{1}, \mathrm{Fe}^{2+}$, and $\mathrm{Mg}$ (apfu) values of biotite for garnetbiotite geothermometers. b - Input $P$ (kbar) and $\mathrm{Al}_{\text {tot }}, \mathrm{Ti}, \mathrm{Fe}_{\text {tot }}$ and $\mathrm{Mg}(a p f u)$ values of clinopyroxene for garnet-clinopyroxene geothermometers. c - Input $P(\mathrm{kbar})$ and $\mathrm{Al}_{\mathrm{tot}}, \mathrm{Fe}^{2+}$ and $\mathrm{Mg}(a p f u)$ values of orthopyroxene for garnet-orthopyroxene geothermometers.

$$
\begin{aligned}
& T 2_{\mathrm{IM} 85}^{\mathrm{Grt} B \mathrm{Bt}}\left({ }^{\circ} \mathrm{C}\right)=\frac{12454+57 P(\mathrm{kbar})+3 \times 454\left(\mathrm{Al}_{\mathrm{Bt}}^{\mathrm{VI}} / 3\right)}{4.662-5.9616 \ln K_{D}+4.5 X_{\mathrm{Ca}}^{\mathrm{Gr}}} \\
& \frac{+3 \times 6767\left(\mathrm{Ti}_{\mathrm{Bt}} / 3\right)+9000 X_{\mathrm{Ca}}^{\mathrm{Grt}}}{4.662-5.9616 \ln K_{D}+4.5 X_{\mathrm{Ca}}^{\mathrm{Grt}}}-273.15
\end{aligned}
$$

Dasgupta et al. (1991) derived a new formulation of garnet-biotite $\mathrm{Fe}^{2+}-\mathrm{Mg}$ exchange geothermometer through statistical regression of the reversed experimental data of Ferry and Spear (1978) taking into consideration the non-ideality terms in garnet and biotite solid solutions. According to Dasgupta et al. (1991), the resultant geothermometer (Eq. 12; see row 28 in Tab. 4) gives consistent results for rocks with a much wider compositional range when compared to the earlier garnet-biotite formulations:

$$
T_{\mathrm{D} 91}^{\mathrm{Grt}-\mathrm{Bt}}\left({ }^{\circ} \mathrm{C}\right)=\frac{4301+3000 X_{\mathrm{Ca}}^{\mathrm{Grt}}+1300 X_{\mathrm{Mn}}^{\mathrm{Grt}}}{1.85-1.9872 \ln K_{D}}
$$


Tab. 4 Garnet-biotite geothermometer estimations by WinGrt program

\begin{tabular}{|c|c|c|c|c|c|c|c|c|c|c|c|c|}
\hline Row & & SG1 & SG2 & SG3 & SG4 & SG5 & SG6 & SG7 & SG8 & SG9 & SG10 & SG11 \\
\hline 1 & $\mathrm{SiO}_{2}$ & 36.700 & 36.500 & 37.900 & 37.100 & 37.300 & 38.300 & 37.400 & 38.400 & 36.900 & 37.400 & 37.800 \\
\hline 2 & $\mathrm{Al}_{2} \mathrm{O}_{3}$ & 20.900 & 20.900 & 19.600 & 20.600 & 21.300 & 20.800 & 21.300 & 21.600 & 20.200 & 21.800 & 20.900 \\
\hline 3 & $\mathrm{FeO}$ & 37.500 & 37.600 & 38.300 & 38.900 & 36.300 & 34.800 & 33.100 & 33.000 & 34.400 & 31.300 & 30.500 \\
\hline 4 & $\mathrm{MgO}$ & 4.400 & 3.800 & 3.400 & 3.300 & 4.300 & 3.100 & 4.000 & 4.000 & 2.710 & 3.800 & 2.970 \\
\hline 5 & $\mathrm{MnO}$ & 0.240 & 0.450 & 0.330 & 0.280 & 0.550 & 0.610 & 0.920 & 0.470 & 0.110 & 0.750 & 0.860 \\
\hline 6 & $\mathrm{CaO}$ & 0.450 & 0.590 & 0.780 & 0.480 & 0.660 & 3.100 & 4.100 & 4.000 & 4.600 & 4.700 & 6.400 \\
\hline 7 & $\sum($ wt. \%) & 100.190 & 99.840 & 100.310 & 100.660 & 100.410 & 100.710 & 100.820 & 101.470 & 98.920 & 99.750 & 99.430 \\
\hline \multicolumn{13}{|c|}{ Garnet recalculation based on 8 cations (apfu) } \\
\hline 8 & $\mathrm{Si}$ & 2.938 & 2.942 & 3.056 & 2.980 & 2.974 & 3.045 & 2.953 & 3.007 & 2.996 & 2.974 & 3.023 \\
\hline 9 & $\mathrm{Al}$ & 1.972 & 1.985 & 1.863 & 1.950 & 2.001 & 1.949 & 1.982 & 1.994 & 1.933 & 2.043 & 1.970 \\
\hline 10 & $\mathrm{Fe}^{3+}$ & 0.153 & 0.131 & 0.025 & 0.089 & 0.051 & 0.000 & 0.112 & 0.000 & 0.076 & 0.009 & 0.000 \\
\hline 11 & $\mathrm{Fe}^{2+}$ & 2.358 & 2.404 & 2.557 & 2.525 & 2.369 & 2.314 & 2.074 & 2.161 & 2.260 & 2.073 & 2.040 \\
\hline 12 & $\mathrm{Mg}$ & 0.525 & 0.457 & 0.409 & 0.395 & 0.511 & 0.367 & 0.471 & 0.467 & 0.328 & 0.450 & 0.354 \\
\hline 13 & $\mathrm{Mn}$ & 0.016 & 0.031 & 0.023 & 0.019 & 0.037 & 0.041 & 0.062 & 0.031 & 0.008 & 0.051 & 0.058 \\
\hline 14 & $\mathrm{Ca}$ & 0.039 & 0.051 & 0.067 & 0.041 & 0.056 & 0.264 & 0.347 & 0.336 & 0.400 & 0.400 & 0.548 \\
\hline 15 & $\sum$ cations & 8.000 & 8.000 & 8.000 & 8.000 & 8.000 & 7.980 & 8.000 & 7.996 & 8.000 & 8.000 & 7.993 \\
\hline \multicolumn{2}{|c|}{ Garnet species } & Alm & Alm & Alm & Alm & Alm & Alm & Alm & Alm & Alm & Alm & Alm \\
\hline \multicolumn{13}{|c|}{ Input recalculated biotite cations (apfu) and pressure (kbar) values for garnet-biotite geothermometers } \\
\hline 16 & $\mathrm{Al}^{\mathrm{VI}}$ & 0.459 & 0.405 & 0.450 & 0.402 & 0.361 & 0.316 & 0.400 & 0.323 & 0.357 & 0.523 & 0.371 \\
\hline 17 & $\mathrm{Ti}$ & 0.046 & 0.096 & 0.082 & 0.098 & 0.111 & 0.101 & 0.074 & 0.104 & 0.097 & 0.061 & 0.098 \\
\hline 18 & $\mathrm{Fe}^{2+}$ & 1.191 & 1.113 & 1.220 & 1.142 & 1.142 & 1.162 & 0.895 & 0.775 & 1.055 & 0.884 & 1.105 \\
\hline 19 & $\mathrm{Mg}$ & 1.219 & 1.341 & 1.126 & 1.281 & 1.296 & 1.308 & 1.540 & 1.741 & 1.409 & 1.444 & 1.335 \\
\hline 20 & $P$ (kbar) & 5 & 5 & 5 & 5 & 5 & 5 & 5 & 5 & 5 & 5 & 5 \\
\hline \multicolumn{13}{|c|}{ Garnet-biotite geothermometers $\left(T,{ }^{\circ} \mathrm{C}\right)$} \\
\hline 21 & $T 1$ & 619 & 535 & 557 & 507 & 582 & 509 & 494 & 432 & 455 & 496 & 513 \\
\hline 22 & $T 2$ & 653 & 540 & 570 & 504 & 602 & 507 & 488 & 411 & 439 & 491 & 512 \\
\hline 23 & $T 3$ & 659 & 547 & 579 & 509 & 610 & 540 & 532 & 451 & 487 & 541 & 580 \\
\hline 24 & $T 4$ & 623 & 562 & 579 & 541 & 597 & 543 & 532 & 483 & 501 & 533 & 546 \\
\hline 25 & $T 5$ & 553 & 478 & 532 & 469 & 517 & 491 & 439 & 368 & 442 & 451 & 513 \\
\hline 26 & $T 6$ & 588 & 469 & 500 & 431 & 526 & 486 & 492 & 401 & 435 & 495 & 543 \\
\hline 27 & $T 7$ & 620 & 493 & 527 & 457 & 546 & 486 & 487 & 401 & 437 & 496 & 522 \\
\hline 28 & $T 8$ & 664 & 566 & 591 & 530 & 624 & 574 & 569 & 494 & 525 & 571 & 621 \\
\hline 29 & $T 9$ & 577 & 551 & 454 & 478 & 584 & 461 & 653 & 707 & 443 & 612 & 464 \\
\hline 30 & $T 10$ & 633 & 568 & 450 & 460 & 633 & 456 & 719 & 769 & 420 & 663 & 475 \\
\hline
\end{tabular}

Notes: The formulae were recalculated to 12 anions and 8 cations; $\mathrm{Fe}^{3+}$ and $\mathrm{Fe}^{2+}$ estimations from total $\mathrm{FeO}$ (wt. \%) contents were carried out on the basis of stoichiometric constraints using Droop's (1987) method; sample numbers SG1 to SG11 from Hoinkes (1986);

Alm = almandine; Thermometers $T 1$ by Thompson (1976), $T 2$ by Ferry and Spear (1978), T3 by Hodges and Spear (1982), $T 4$ by Perchuk and Lavrent'eva (1983), $T 5$ by Ganguly and Saxena (1984), T6 and $T 7$ by Indares and Mortignale (1985), $T 8$ by Dasgupta et al. (1991), T9 by Bhattacharya et al. (1992) using the updated mixing parameters of Hackler and Wood (1989), T10 by Bhattacharya et al. (1992) using the updated mixing parameters of Ganguly and Saxena (1984)

$$
\begin{aligned}
& \frac{-495\left(X_{\mathrm{Mg}}^{\mathrm{Grt}}-X_{\mathrm{Fe}}^{\mathrm{Grt}}\right)-3595\left(\mathrm{Al}_{\mathrm{Bt}}^{\mathrm{VI}} / 3\right)-4423\left(\mathrm{Ti}_{\mathrm{Bt}} / 3\right)}{1.85-1.9872 \ln K_{D}} \\
& \frac{+1073\left(\left(\mathrm{Mg}_{\mathrm{Bt}} / 3\right)-\left(\mathrm{Fe}_{\mathrm{Bt}} / 3\right)\right)+24.6 P(\mathrm{kbar})}{1.85-1.9872 \ln K_{D}}-273.15
\end{aligned}
$$

Bhattacharya et al. (1992) attempted a theoretical analysis of the experimentally observed $P-T-X(\mathrm{Mg}-\mathrm{Fe})$ relationship in the biotite-garnet system using the updated mixing parameters for the pyrope-almandine asymmetric regular solution by Ganguly and Saxena (1984) and Hackler and Wood (1989). Thus, they proposed two new garnet-biotite formulations (see rows 29-30 in
Tab. 4) for application to natural rocks including garnet and biotite pairs:

$$
\begin{aligned}
& T 1_{\mathrm{B} 92-\mathrm{Gs} 84}^{\mathrm{Grt}}\left({ }^{\circ} \mathrm{C}\right)=\frac{13538+19.3 P(\mathrm{kbar})-\left\{837\left(X_{\mathrm{Mg}}^{\mathrm{Grt}}\right)^{2}\right.}{6.778+8.3143 \ln K_{D}+6.276 X_{\mathrm{Ca}}^{\mathrm{Grt}}\left(1-X_{\mathrm{Mn}}^{\mathrm{Grt}}\right)} \\
& \frac{-10460\left(X_{\mathrm{Fe}}^{\mathrm{Grt}}\right)^{2}-13807 X_{\mathrm{Ca}}^{\mathrm{Grt}}\left(1-X_{\mathrm{Mn}}^{\mathrm{Grt}}\right)}{6.778+8.3143 \ln K_{D}+6.276 X_{\mathrm{Ca}}^{\mathrm{Grt}}\left(1-X_{\mathrm{Mn}}^{\mathrm{Grt}}\right)} \\
& \frac{\left.+19246 X_{\mathrm{Fe}}^{\mathrm{Grt}} X_{\mathrm{Mg}}^{\mathrm{Grt}}\left(1-X_{\mathrm{Mn}}^{\mathrm{Grt}}\right)+5649 X_{\mathrm{Ca}}^{\mathrm{Grt}}\left(X_{\mathrm{Mg}}^{\mathrm{Grt}}-X_{\mathrm{Fe}}^{\mathrm{Grt}}\right)\right\}}{6.778+8.3143 \ln K_{D}+6.276 X_{\mathrm{Ca}}^{\mathrm{Grt}}\left(1-X_{\mathrm{Mn}}^{\mathrm{Grt}}\right)} \\
& \quad+7972\left(2 X_{\mathrm{Mg}}^{\mathrm{Bt}}-1\right) \\
& \frac{6.778+8.3143 \ln K_{D}+6.276 X_{\mathrm{Ca}}^{\mathrm{Grt}}\left(1-X_{\mathrm{Mn}}^{\mathrm{Grt}}\right)}{2}-273.15
\end{aligned}
$$




$$
\begin{aligned}
& T 2_{\mathrm{B} 92 \_\mathrm{HW} 84}^{\mathrm{Grt}-\mathrm{Bt}}\left({ }^{\circ} \mathrm{C}\right)=\frac{20286+19.3 P(\mathrm{kbar})-\left\{2080\left(X_{\mathrm{Mg}}^{\mathrm{Git}}\right)^{2}\right.}{13.138+8.3143 \ln K_{D}+6.276 X_{\mathrm{Ca}}^{\mathrm{Grt}}\left(1-X_{\mathrm{Mn}}^{\mathrm{Git}}\right)} \\
& \frac{-6350\left(X_{\mathrm{Fe}}^{\mathrm{Git}}\right)^{2}-13807 X_{\mathrm{Ca}}^{\mathrm{Grt}}\left(1-X_{\mathrm{Mn}}^{\mathrm{Grt}}\right)}{13.138+8.3143 \ln K_{D}+6.276 X_{\mathrm{Ca}}^{\mathrm{Grt}}\left(1-X_{\mathrm{Mn}}^{\mathrm{Git}}\right)} \\
& \frac{\left.+8540 X_{\mathrm{Fe}}^{\mathrm{Git}} X_{\mathrm{Mg}}^{\mathrm{Git}}\left(1-X_{\mathrm{Mn}}^{\mathrm{Git}}\right)+4215 X_{\mathrm{Ca}}^{\mathrm{Grt}}\left(X_{\mathrm{Mg}}^{\mathrm{Grt}}-X_{\mathrm{Fe}}^{\mathrm{Grtt}}\right)\right\}}{13.138+8.3143 \ln K_{D}+6.276 X_{\mathrm{Ca}}^{\mathrm{Grt}}\left(1-X_{\mathrm{Mn}}^{\mathrm{Grt}}\right)} \\
& \frac{+4441\left(2 X_{\mathrm{Mg}}^{\mathrm{Bt}}-1\right)}{13.138+8.3143 \ln K_{D}+6.276 X_{\mathrm{Ca}}^{\mathrm{Git}}\left(1-X_{\mathrm{Mn}}^{\mathrm{Grt}}\right)}-273.15(14
\end{aligned}
$$

where $K_{\mathrm{D}}=\mathrm{FeMg}_{\mathrm{Grt}} / \mathrm{FeMg}_{\mathrm{Bt}} ; \mathrm{FeMg}_{\mathrm{Grt}}=\mathrm{Fe}_{\mathrm{Grt}} / \mathrm{Mg}_{\mathrm{Grt}}$; $\mathrm{FeMg}_{\mathrm{Bt}}=\mathrm{Mg}_{\mathrm{Bt}} / \mathrm{Fe}_{\mathrm{Bt}} ; \mathrm{Fe}_{\mathrm{Grt}}=\mathrm{Fe}^{2+} /\left(\mathrm{Mg}+\mathrm{Fe}^{2+}+\mathrm{Mn}+\mathrm{Ca}\right) ;$ $\mathrm{Mg}_{\mathrm{Grt}}=\mathrm{Mg} /\left(\mathrm{Mg}+\mathrm{Fe}^{2+}+\mathrm{Mn}+\mathrm{Ca}\right) ; \mathrm{Mg}_{\mathrm{Bt}}=$ octahedral $\mathrm{Mg}$ content (apfu) and $\mathrm{Fe}_{\mathrm{Bt}}=$ octahedral $\mathrm{Fe}^{2+}$ content (apfu).

Consequently, the garnet-biotite geothermometer appears to estimate the temperature conditions of rocks metamorphosed under high- $T$ greenschist and amphibolite facies fairly well. However, due to the retrograde $\mathrm{Fe}^{2+}-\mathrm{Mg}$ exchange in the upper amphibolite and granulite facies, the garnet-biotite geothermometers may give anomalously low temperatures, if garnet rims and adjacent biotites in thin sections are analyzed. In this case, reasonable estimates of maximum prograde temperatures may be obtained by using the garnet core and matrix biotite compositions, provided garnet and biotite compositions were once in chemical equilibrium (Bucher and Grapes 2011).

\subsubsection{Garnet-clinopyroxene geothermometers}

Garnet-clinopyroxene geothermometers which were in general calibrated in the range of $600-1500^{\circ} \mathrm{C}$, have been widely used by petrologists and geochemists. Without consideration of the non-ideality of garnet, Råheim and Green (1974) experimentally calibrated $K_{\mathrm{D}}$ as a function of pressure and temperature for basaltic rocks that crystallized to eclogite at $20-40 \mathrm{kbar}$ and $600-1400^{\circ} \mathrm{C}$, respectively. Based on the experimental results, they proposed an empirical garnet-clinopyroxene geothermometer (see row 25 in Tab. 5) that could only be used for rocks of basaltic composition:

$T_{\mathrm{RG} 74}^{\mathrm{Grt}-\mathrm{Cpx}}\left({ }^{\circ} \mathrm{C}\right)=\frac{3686+28.35 P(\mathrm{kbar})}{2.33+\ln K_{D}}-273.15$

where $K_{\mathrm{D}}=\left(\mathrm{Fe}^{2+} / \mathrm{Mg}\right)^{\mathrm{Grt}} /\left(\mathrm{Fe}^{2+} / \mathrm{Mg}\right)^{\mathrm{Cpx}}$ for the following garnet-clinopyroxene $\mathrm{Fe}^{2+}-\mathrm{Mg}$ exchange reaction:

$$
\begin{aligned}
& \underset{\text { pyrope }}{1 / 3 \mathrm{Mg}_{3} \mathrm{Al}_{2} \mathrm{Si}_{3} \mathrm{O}_{12}}+\underset{\mathrm{CaFeSi}_{2} \mathrm{O}_{6}}{\text { hedenbergite }}=1 / 3 \mathrm{Fe}^{2+}{ }_{3} \mathrm{Al}_{2} \mathrm{Si}_{3} \mathrm{O}_{12} \\
& \text { almandine }
\end{aligned}
$$

Ellis and Green (1979) carried out a series of experiments for basaltic compositions within the simple $\mathrm{CaO}-\mathrm{MgO}-\mathrm{FeO}-\mathrm{Al}_{2} \mathrm{O}_{3}-\mathrm{SiO}_{2}$ system that crystallized to garnet-clinopyroxene-bearing mineral assemblages at $24-30 \mathrm{kbar}$ and $750-1300^{\circ} \mathrm{C}$. They experimentally proven that the non-ideality of $\mathrm{Ca}$ content in garnet is important, and thus incorporated a non-ideal term for $\mathrm{Ca}$ in garnet into their formulation. Ellis and Green (1979) established $K_{\mathrm{D}}$ as a function of pressure, temperature, and molar fraction of grossular $\left(X_{\mathrm{Ca}}^{\text {Grt }}\right)$, proposing an empirical geothermometer (see row 26 in Tab. 5) applicable to garnet-clinopyroxene-bearing rocks from a wide range of geological environments:

$$
T_{\mathrm{EG} 79}^{\mathrm{Grt}-\mathrm{Cx}}\left({ }^{\circ} \mathrm{C}\right)=\frac{3104 X_{\mathrm{Ca}}^{\mathrm{Grt}}+3030+10.86 P(\mathrm{kbar})}{\ln K_{D}+1.9034}-273.15
$$

Being an improved version of the first garnet-clinopyroxene geothermometer (i.e. Råheim and Green 1974), Eq. 17 and its different calibrations found a broad application in the field of metamorphic petrology.

Ganguly (1979) developed a semiempirical theoretical formulation of garnet-clinopyroxene geothermometer (see row 27 in Tab. 5) on the basis of thermodynamic mixing data of garnet solid solutions with grossular and spessartine components:

$T_{\mathrm{G} 79}^{\mathrm{Grt}-\mathrm{Cpx}}\left({ }^{\circ} \mathrm{C}\right)=\frac{4100+1586 X_{\mathrm{Ca}}^{\mathrm{Grt}}+11.07 P(\mathrm{kbar})}{2.4+\ln K_{D}}-273.15$

for $T\left({ }^{\circ} \mathrm{C}\right) \geq 1060$

$T_{\mathrm{G} 79}^{\mathrm{Grt}-\mathrm{Cpx}}\left({ }^{\circ} \mathrm{C}\right)=\frac{4801+1586 X_{\mathrm{Ca}}^{\mathrm{Grt}}+11.07 P(\mathrm{kbar})}{2.93+\ln K_{D}}-273.15$

for $T\left({ }^{\circ} \mathrm{C}\right) \leq 1060$

WinGrt estimates temperatures by Ganguly's (1979) geothermometer using the Eq. 18a as a default. However, by clicking the Use Ganguly's (1979) Garnet-Clinopyroxene Geothermometer for $T\left({ }^{\circ} \mathrm{C}\right) \leq 1060$ option from the Calculate menu, the program calculates Ganguly's (1979) geothermometer using the Eq. 18b.

The process of calibration of a geothermobarometer should involve the determination of the parameters, as well as the uncertainties. Powell (1985) proposed a new garnet-clinopyroxene geothermometer (see row 28 in Tab. 5), derived using a rigorous statistical consideration:

$$
T_{\mathrm{P} 85}^{\mathrm{Grt}-\mathrm{Cpx}}\left({ }^{\circ} \mathrm{C}\right)=\frac{2790+3140 X_{\mathrm{Ca}}^{\mathrm{Grt}}+10 P(\mathrm{kbar})}{1.735+\ln K_{D}}-273.15
$$

Using the previous experimental data on the partitioning of $\mathrm{Fe}^{2+}$ and $\mathrm{Mg}$ between garnet and clinopyroxene (e.g. Råheim and Green 1974; Ellis and Green 1979), a new expression for the garnet-clinopyroxene geother- 
mometer (Eq. 20; see row 29 in Tab. 5), including a curvilinear relationship between $\ln K_{\mathrm{D}}$ and molar fraction of grossular $\left(X_{\mathrm{Ca}}^{\mathrm{Grt}}\right)$, has been derived by Krogh (1988). It was used to calculate equilibration temperatures for samples of eclogites and associated omphacite-bearing high-pressure gneisses from the Tromsø nappe complex within the northern Scandinavian Caledonides.

$$
T_{\mathrm{K} 88}^{\mathrm{Grt}-\mathrm{Cpx}}\left({ }^{\circ} \mathrm{C}\right)=\frac{-6173\left(X_{\mathrm{Ca}}^{\mathrm{Grt}}\right)^{2}+6731 X_{\mathrm{Ca}}^{\mathrm{Grt}}+1879+10 P(\mathrm{kbar})}{1.393+\ln K_{D}}
$$$$
-273.15
$$

Taking into account the significant influence of garnet $\mathrm{Mg}$ number (Mg\#) on the garnet-clinopyroxene $\mathrm{Fe}^{2+} / \mathrm{Mg}$ partition coefficient $\left(K_{\mathrm{D}}\right), \mathrm{Ai}(1994)$ used a comprehensive experimental dataset and developed an empirical geothermometer (see row 30 in Tab. 5) for the lower crustal rocks including garnet amphibolites, granulites and eclogites as well as for the upper mantle eclogite and lherzolite xenoliths in kimberlites and mineral inclusions in diamonds:

$$
\begin{aligned}
& T_{\mathrm{K} 88}^{\mathrm{Git}-\mathrm{Cpx}}\left({ }^{\circ} \mathrm{C}\right)=\frac{-1629\left(X_{\mathrm{Ca}}^{\mathrm{Git}}\right)^{2}+3648.55 X_{\mathrm{Ca}}^{\mathrm{Grt}}-6.59 \mathrm{Mg} \#^{\mathrm{Git}}}{1.076+\ln K_{D}} \\
& \frac{+1987.98+17.66 P(\mathrm{kbar})}{1.076+\ln K_{D}}-273.15
\end{aligned}
$$

where $\mathrm{Mg} \#^{\mathrm{Grt}}=100 \mathrm{Mg} /\left(\mathrm{Mg}+\mathrm{Fe}^{2+}\right)$.

Ganguly et al. (1996) reported new experimental data involving primarily $\mathrm{Mg}-\mathrm{Ca}-\mathrm{Mn}$ and $\mathrm{Fe}-\mathrm{Mg}-\mathrm{Ca}-\mathrm{Mn}$ and to a limited extent $\mathrm{Fe}-\mathrm{Mg}-\mathrm{Ca}$ and $\mathrm{Mg}-\mathrm{Ca}$ garnet compositions. They showed phase diagrams to illustrate unmixing in the binary and ternary garnet solid solutions, presented thermodynamically based formulations, and applied the solution model to develop compositional corrections for the garnet-orthopyroxene and garnet-clinopyroxene $\mathrm{Fe}^{2+}-\mathrm{Mg}$ exchange geothermometers (see row 31 in Tab. 5):

$$
T_{\mathrm{G} 96}^{\mathrm{Grt}-\mathrm{Cpx}}\left({ }^{\circ} \mathrm{C}\right)=\frac{4100+11.07 P(\mathrm{kbar})-\mathrm{A}}{2.4+\ln K_{D}+\mathrm{B}}-273.15
$$

where

$$
\begin{aligned}
& \mathrm{A}=\frac{(21627+12 P)\left(-2 X_{\mathrm{Ca}}^{\mathrm{Grt}} X_{\mathrm{Mg}}^{\mathrm{Grt}}+a\right)}{8.3144} \\
& \frac{+(9834+58 P)\left(\left(-X_{\mathrm{Ca}}^{\mathrm{Grt}}\right)^{2}+a\right)+873\left(2 X_{\mathrm{Ca}}^{\mathrm{Grt}} X_{\mathrm{Fe}}^{\mathrm{Grt}}+a\right)}{8.3144} \\
& \frac{-(6733+30 P)\left(\left(-X_{\mathrm{Ca}}^{\mathrm{Grt}}\right)^{2}+b\right)+(12083-539) X_{\mathrm{Mn}}^{\mathrm{Grt}}}{8.3144}, \\
& \mathrm{~B}=\frac{-5.78\left(-2 X_{\mathrm{Ca}}^{\mathrm{Grt}} X_{\mathrm{Mg}}^{\mathrm{Grt}}+a\right)-5.78\left(-\left(X_{\mathrm{Ca}}^{\mathrm{Grt}}\right)^{2}+a\right)}{8.3144} \\
& \frac{-1.69\left(2 X_{\mathrm{Ca}}^{\mathrm{Grt}} X_{\mathrm{Fe}}^{\mathrm{Grt}}+100\right)+1.69\left(\left(-X_{\mathrm{Ca}}^{\mathrm{Grt}}\right)^{2}+b\right)+7.69 X_{\mathrm{Mn}}^{\mathrm{Grt}}}{8.3144},
\end{aligned}
$$

$a=0.5 X_{\mathrm{Ca}}^{\mathrm{Git}}\left(X_{\mathrm{Mg}}^{\mathrm{Grt}}-X_{\mathrm{Fe}}^{\mathrm{Git}}-X_{\mathrm{Mn}}^{\mathrm{Git}}\right)$ and $b=0.5 X_{\mathrm{Ca}}^{\mathrm{Git}}\left(X_{\mathrm{Mg}}^{\mathrm{Git}}-X_{\mathrm{Fe}}^{\mathrm{Git}}\right.$ $\left.+X_{\mathrm{Mn}}^{\mathrm{Grt}}\right)$.

They also argued that their garnet-clinopyroxene geothermometer should not be applied to eclogites unless these are mantle-derived (i.e. $T>1000^{\circ} \mathrm{C}$ ).

Liu (1998) carried out a series of experimental studies for $\mathrm{Fe}-\mathrm{Mg}$ exchange equilibrium between garnet and clinopyroxene at $600-950{ }^{\circ} \mathrm{C}, 0.8-3.0 \mathrm{GPa}$, and fayalite-quartz-magnetite buffer $\left(f \mathrm{O}_{2}\right)$ conditions in the basalt- $\mathrm{H}_{2} \mathrm{O}$ system. He formulated a new garnet-clinopyroxene geothermometer (see row 32 in Tab. 5) applicable to rocks in amphibolite, granulite, and eclogite terranes: $T_{\mathrm{L} 98}^{\mathrm{Grt}-\mathrm{Cpx}}\left({ }^{\circ} \mathrm{C}\right)=\frac{3820}{1+0.13 \times(2.2-P(\mathrm{GPa})) \ln K_{D}+1.828}-273.15$

Applying the multiple regression analysis technique on a large experimental data set and natural high-Mn granulites, Krogh-Ravna (2000) proposed a new garnetclinopyroxene geothermometer (see row 33 in Tab. 5):

$$
\begin{aligned}
& T_{\mathrm{KR} 00}^{\mathrm{Grt}-\mathrm{Cpx}}\left({ }^{\circ} \mathrm{C}\right)=\frac{1939.9+3270 X_{\mathrm{Ca}}^{\mathrm{Grt}}-1396\left(X_{\mathrm{Ca}}^{\mathrm{Grt}}\right)^{2}+3319 X_{\mathrm{Mn}}^{\mathrm{Grt}}}{1.223+\ln K_{D}} \\
& \frac{-3535 X_{\mathrm{Mn}}^{\mathrm{Grt}}+1105 X_{\mathrm{Mg} \#}^{\mathrm{Grt}}-3561\left(X_{\mathrm{Mg} \#}^{\mathrm{Grt}}\right)^{2}+2324\left(X_{\mathrm{Mg} \#}^{\mathrm{Grt}}\right)^{3}}{1.223+\ln K_{D}} \\
& \frac{+169.4 P(\mathrm{GPa})}{1.223+\ln K_{D}}-273.15
\end{aligned}
$$

Based on the compilation of experimental data, the author stressed that the amount of jadeite component in clinopyroxene does not seem to affect the $\mathrm{Fe}-\mathrm{Mg}$ distribution coefficient between garnet and clinopyroxene. In addition to a significant dependence between the distribution coefficient $K_{\mathrm{D}}, X_{\mathrm{Ca}}^{\mathrm{Grt}}$ and $X_{\mathrm{Mg}}^{\mathrm{Grt}}, \mathrm{Krogh}$-Ravna's (2000) empirical formulation also considers the effect of $X_{\mathrm{Mn}}^{\mathrm{Grt}}$ in temperature estimation.

Over 300 garnet-clinopyroxene pairs from published experimental data were used by Nakamura (2009) to develop a new formulation of the garnet-clinopyroxene geothermometer (see row 34 in Tab. 5) based on statistical analysis technique:

$$
T_{\mathrm{N} 09}^{\mathrm{Grt}-\mathrm{Cpx}}\left({ }^{\circ} \mathrm{C}\right)=\frac{\mathrm{A}-\mathrm{B}-\mathrm{C}}{\mathrm{D}}-273.15
$$

where

$\mathrm{A}=2784+14.52 P(\mathrm{kbar})+(2601+1.44 P(\mathrm{kbar}))$

$\times\left(2 X_{\mathrm{Ca}}^{\mathrm{Grt}} X_{\mathrm{Mg}}^{\mathrm{Grt}}-a\right)+(1183+6.98 P(\mathrm{kbar}))\left(\left(X_{\mathrm{Ca}}^{\mathrm{Grt}}\right)^{2}-a\right)$,

$\mathrm{B}=105\left(2 X_{\mathrm{Ca}}^{\mathrm{Grt}} X_{\mathrm{Fe}}^{\mathrm{Grt}}+b\right)+(814.6+3.61 P(\mathrm{kbar}))$

$\times\left(\left(X_{\mathrm{Ca}}^{\mathrm{Grt}}\right)^{2}+b\right)-(254.6+8.42 P(\mathrm{kbar}))\left(2 X_{\mathrm{Mg}}^{\mathrm{Grt}} X_{\mathrm{Fe}}^{\mathrm{Grt}}\right.$

$\left.-\left(X_{\mathrm{Fe}}^{\mathrm{Grt}}\right)^{2}+c\right)$, 
$\mathrm{C}=83.6\left(\left(X_{\mathrm{Mg}}^{\mathrm{Grt}}\right)^{2}-2 X_{\mathrm{Mg}}^{\mathrm{Grt}} X_{\mathrm{Fe}}^{\mathrm{Grt}}+c\right)+1388 X_{\mathrm{Mn}}^{\mathrm{Grt}}-462\left(X_{\mathrm{Mg}}^{\mathrm{Grt}}-X_{\mathrm{Fe}}^{\mathrm{Grt}}\right)$,

$\mathrm{D}=\ln K_{\mathrm{D}}+1.431+0.695\left(2 X_{\mathrm{Ca}}^{\mathrm{Grt}} X_{\mathrm{Mg}}^{\mathrm{Grt}}+\left(X_{\mathrm{Ca}}^{\mathrm{Grt}}\right)^{2}-2 a\right)+0.203\left(\left(X_{\mathrm{Ca}}^{\mathrm{Grt}}\right)^{2}\right.$

$\left.-2 X_{\mathrm{Ca}}^{\mathrm{Grt}} X_{\mathrm{Fe}}^{\mathrm{Grt}}\right)+0.922 X_{\mathrm{Mn}}^{\mathrm{Grt}}$,

$a=0.5 X_{\mathrm{Ca}}^{\mathrm{Grt}}\left(X_{\mathrm{Mg}}^{\mathrm{Grt}}-X_{\mathrm{Fe}}^{\mathrm{Grt}}-X_{\mathrm{Mn}}^{\mathrm{Grt}}\right), b=0.5 X_{\mathrm{Ca}}^{\mathrm{Grt}}\left(X_{\mathrm{Mg}}^{\mathrm{Grt}}-X_{\mathrm{Fe}}^{\mathrm{Grt}}\right.$

$\left.+X_{\mathrm{Mn}}^{\mathrm{Grt}}\right)$ and $c=0.5\left(X_{\mathrm{Ca}}^{\mathrm{Grt}}+X_{\mathrm{Mn}}^{\mathrm{Grt}}\right)\left(X_{\mathrm{Mg}}^{\mathrm{Grt}}-X_{\mathrm{Fe}}^{\mathrm{Grt}}\right)$.

According to Nakamura (2009), tests of his garnetclinopyroxene geothermometer for the accumulated data set are concordant with the experimental temperatures over the whole range of the experimental temperatures be- tween 800 and $1820^{\circ} \mathrm{C}$, with a standard deviation of $74{ }^{\circ} \mathrm{C}$

\subsubsection{Garnet-orthopyroxene geothermometers}

Compared to the garnet-clinopyroxene geothermometry, the temperature dependence of the $\mathrm{Fe}^{2+}-\mathrm{Mg}$ distribution between garnet and orthopyroxene is less pronounced, but recognized as a potential indicator of the $P-T$ conditions of formation for a variety of natural assemblages,

Tab. 5 Garnet-clinopyroxene geothermometer estimations by WinGrt program

\begin{tabular}{|c|c|c|c|c|c|c|}
\hline Row & & SG1 & SG2 & SG3 & SG4 & SG5 \\
\hline 1 & $\mathrm{SiO}_{2}$ & 40.230 & 39.920 & 40.090 & 40.060 & 40.480 \\
\hline 2 & $\mathrm{TiO}_{2}$ & 0.550 & 0.760 & 0.520 & 0.660 & 0.530 \\
\hline 3 & $\mathrm{Al}_{2} \mathrm{O}_{3}$ & 21.870 & 21.450 & 22.010 & 21.820 & 22.380 \\
\hline 4 & $\mathrm{FeO}$ & 19.030 & 19.170 & 18.480 & 18.590 & 16.000 \\
\hline 5 & $\mathrm{MgO}$ & 10.070 & 9.650 & 10.340 & 10.620 & 12.700 \\
\hline 6 & $\mathrm{MnO}$ & 0.400 & 0.450 & 0.410 & 0.460 & 0.410 \\
\hline 7 & $\mathrm{CaO}$ & 7.270 & 8.090 & 7.740 & 7.560 & 7.350 \\
\hline 8 & $\mathrm{Na}_{2} \mathrm{O}$ & 0.000 & 0.000 & 0.000 & 0.000 & 0.000 \\
\hline 9 & $\sum$ (wt. \%) & 99.420 & 99.490 & 99.590 & 99.770 & 99.850 \\
\hline \multicolumn{7}{|c|}{ Garnet recalculation based on 8 cations $(a p f u)$} \\
\hline 10 & $\mathrm{Si}$ & 3.036 & 3.025 & 3.018 & 3.013 & 3.001 \\
\hline 11 & $\mathrm{Ti}$ & 0.031 & 0.043 & 0.029 & 0.037 & 0.030 \\
\hline 12 & $\mathrm{Al}$ & 1.945 & 1.916 & 1.953 & 1.934 & 1.956 \\
\hline 14 & $\mathrm{Fe}^{2+}$ & 1.201 & 1.215 & 1.164 & 1.169 & 0.992 \\
\hline 15 & $\mathrm{Mg}$ & 1.133 & 1.090 & 1.161 & 1.191 & 1.404 \\
\hline 16 & $\mathrm{Mn}$ & 0.026 & 0.029 & 0.026 & 0.029 & 0.026 \\
\hline 17 & $\mathrm{Ca}$ & 0.588 & 0.657 & 0.624 & 0.609 & 0.584 \\
\hline 18 & $\mathrm{Na}$ & 0.000 & 0.000 & 0.000 & 0.000 & 0.000 \\
\hline 19 & $\sum$ cations & 7.960 & 7.974 & 7.976 & 7.983 & 7.992 \\
\hline Garn & ies & Alm & Alm & Alm & Prp & Prp \\
\hline \multicolumn{7}{|c|}{ Input clinopyroxene cations $(a p f u)$ and pressure (kbar) for geothermometer estimations } \\
\hline 20 & $\mathrm{Al}_{\text {tot }}$ & 0.437 & 0.468 & 0.465 & 0.376 & 0.388 \\
\hline 21 & $\mathrm{Fe}_{\text {tot }}$ & 0.191 & 0.221 & 0.228 & 0.216 & 0.202 \\
\hline 22 & $\mathrm{Fe}^{2+}$ & 0.191 & 0.221 & 0.228 & 0.216 & 0.202 \\
\hline 23 & $\mathrm{Mg}$ & 0.536 & 0.507 & 0.538 & 0.593 & 0.664 \\
\hline 24 & $P$ (kbar) & 25 & 25 & 25 & 25 & 25 \\
\hline 25 & $T 1$ & 1012 & 1071 & 1104 & 1050 & 1112 \\
\hline 26 & $T 2$ & 1037 & 1129 & 1157 & 1085 & 1149 \\
\hline 27 & $T 3$ & 1072 & 1142 & 1171 & 1112 & 1172 \\
\hline 28 & $T 4$ & 1025 & 1122 & 1152 & 1076 & 1143 \\
\hline 29 & $T 5$ & 1026 & 1146 & 1178 & 1086 & 1160 \\
\hline 30 & $T 6$ & 1007 & 1137 & 1170 & 1067 & 1129 \\
\hline 31 & $T 7$ & 1077 & 1144 & 1174 & 1118 & 1182 \\
\hline 32 & $T 8$ & 1003 & 1123 & 1152 & 1060 & 1115 \\
\hline 33 & $T 9$ & 1087 & 1183 & 1221 & 1140 & 1218 \\
\hline 34 & $T 10$ & 1055 & 1126 & 1165 & 1101 & 1175 \\
\hline
\end{tabular}

Notes: The formulae were recalculated to 12 anions and 8 cations; sample numbers SG1 to SG5 from Nakamura and Hirajima (2005);

Alm = almandine, Prp = pyrope; Garnet-clinopyroxene geothermometer of $T 1$ by Råheim and Green (1974), T2 by Ellis and Green (1979), T3 by Ganguly (1979), T4 by Powell (1985), T5 by Krogh (1988), T6 by Ai (1994), 77 by Ganguly et al. (1996), T8 by Liu (1998), T9 by Krogh-Ravna (2000), T10 by Nakamura (2009). 
especially for basic granulites and charnockites that formed at granulite-facies metamorphic conditions in the Earth's upper mantle. Garnet-orthopyroxene pairs coexist at $700-900{ }^{\circ} \mathrm{C}$ (rarely up to $1000^{\circ} \mathrm{C}$ ) and $4-10 \mathrm{kbar}$ in rocks including charnockites, basic granulites, aluminous granulites, pyroxene hornfelses and metamorphic iron formations. Coexisting garnet-orthopyroxene pairs are also found in mantle-derived lherzolites, eclogites and peridotites at $>800^{\circ} \mathrm{C}$ and $10 \mathrm{kbar}$ (Lal 1993).

Dahl (1980) studied the garnet-clinopyroxene and garnet-orthopyroxene compositions in diverse metamorphic lithologies from two small areas in the Ruby Range, southwestern Montana, and proposed an empirical calibration of garnet-orthopyroxene geothermometer (see row 27 in Tab. 6) based on a limited mineral-pair analysis by using the multiple linear regression technique:

$$
\begin{aligned}
& T_{\mathrm{D} 80}^{\mathrm{Grt}-\mathrm{Opx}}\left({ }^{\circ} \mathrm{C}\right)=\frac{1391+1509\left(X_{\mathrm{Fe}}^{\mathrm{Grt}}-X_{\mathrm{Mg}}^{\mathrm{Grt}}\right)+2810 X_{\mathrm{Ca}}^{\mathrm{Grt}}}{1.987 \ln K_{D}} \\
& \frac{+2855 X_{\mathrm{Mn}}^{\mathrm{Grt}}}{1.987 \ln K_{D}}-273.15
\end{aligned}
$$

where $K_{\mathrm{D}}$ is $\left(\mathrm{Fe}^{2+} / \mathrm{Mg}\right)^{\mathrm{Grt}} /\left(\mathrm{Fe}^{2+} / \mathrm{Mg}\right)^{\mathrm{Opx}}$ for the following garnet-orthopyroxene $\mathrm{Fe}^{2+}-\mathrm{Mg}$ exchange reaction:

$$
\begin{aligned}
& 1 / 2 \mathrm{Fe}_{2} \mathrm{SiO}_{6}+1 / 3 \mathrm{Mg}_{3} \mathrm{Al}_{2} \mathrm{Si}_{3} \mathrm{O}_{12}=\underset{2}{1 / 2 \mathrm{Mg}_{2} \mathrm{Si}_{2} \mathrm{O}_{6}} \\
& \text { ferrosilite pyrope } \\
& +1 / 3 \mathrm{Fe}_{3} \mathrm{Al}_{2} \mathrm{Si}_{3} \mathrm{O}_{12} \\
& \quad \text { almandine }
\end{aligned}
$$

Neglecting the non-ideality of the Fe-Mg substitution in garnet and orthopyroxene phases and the effect of pressure, Raith et al. (1983) developed an empirical garnet-orthopyroxene geothermometer (see row 28 in Tab. 6) using the compositional data from garnet lherzolite nodules in the granulite-facies terrain of South India:

$$
T_{\mathrm{R} 83}^{\mathrm{Grt}-\mathrm{Opx}}\left({ }^{\circ} \mathrm{C}\right)=\frac{1684}{0.334+\ln K_{D}}-273.15
$$

The partitioning of Fe and $\mathrm{Mg}$ between garnet and aluminous orthopyroxene was experimentally investigated by Harley (1984) within the $P-T$ range of 5-30 kbar and $800-1200^{\circ} \mathrm{C}$ in the $\mathrm{FeO}-\mathrm{MgO}-\mathrm{Al}_{2} \mathrm{O}_{3}-\mathrm{SiO}_{2}$ and $\mathrm{CaO}-\mathrm{FeO}-\mathrm{MgO}-\mathrm{Al}_{2} \mathrm{O}_{3}-\mathrm{SiO}_{2}$ systems. The calibration of garnet-orthopyroxene geothermometer (see row 29 in Tab. 6) is applicable to garnet peridotites and granulites:

$$
T_{\mathrm{H} 84}^{\mathrm{Grt}-\mathrm{Opx}}\left({ }^{\circ} \mathrm{C}\right)=\frac{3740+1400 X_{\mathrm{Ca}}^{\mathrm{Grt}}+22.86 P(\mathrm{kbar})}{1.9872 \ln K_{D}+1.96}-273.15(29)
$$

Although the accuracy and precision of Harley's (1984) calibration are limited due to large relative errors in the experimental and natural-rock data, it was however pointed out that this geothermometer yields reasonable temperature estimates for a variety of natural samples.
Taking into account the enthalpy, entropy and the volume data of $\mathrm{Mg}$ and $\mathrm{Fe}^{2+}$ end-member components in garnet and orthopyroxene solid solutions, a new $\mathrm{Fe}^{2+}-\mathrm{Mg}$ exchange geothermometer (see row 30 in Tab. 6) for coexisting orthopyroxene-garnet pairs was formulated by Sen and Bhattacharya (1984) for granulite-facies quartzofeldspathic and basic lithologies:

$T_{\mathrm{SB} 84}^{\mathrm{Grt}-\mathrm{Opx}}\left({ }^{\circ} \mathrm{C}\right)=\frac{2713+22 P(\mathrm{kbar})+3300 X_{\mathrm{Ca}}^{\mathrm{Git}}+195\left(X_{\mathrm{Fe}}^{\mathrm{Grt}}-X_{\mathrm{Mg}}^{\mathrm{Grt}}\right)}{-1.9872 \ln K_{D}+0.787+1.5 X_{\mathrm{Ca}}^{\mathrm{Grt}}}$

$-273.15$

where $K_{\mathrm{D}}=\left(\mathrm{Fe}^{2+} / \mathrm{Mg}\right)^{\mathrm{Opx}} /\left(\mathrm{Fe}^{2+} / \mathrm{Mg}\right)^{\mathrm{Grt}}$.

Based on an experimental study for garnet and orthopyroxene pairs, Lee and Ganguly (1984) derived an exchange $\mathrm{Fe}^{2+}-\mathrm{Mg}$ geothermometer (see row 31 in Tab. 6) with an empirical adjustment from natural data set in terms of $\mathrm{Ca}$ and $\mathrm{Mn}$ contents:

$$
T_{\mathrm{LG} 84}^{\mathrm{Grt}-\mathrm{Opx}}\left({ }^{\circ} \mathrm{C}\right)=\frac{2187+1510\left(X_{\mathrm{Ca}}^{\mathrm{Grt}}-X_{\mathrm{Mn}}^{\mathrm{Grt}}\right)+8.6 P(\mathrm{kbar})}{\ln K_{D}+1.071}
$$

$-273.15$

Using thermodynamic parameters listed in their table and corresponding activity expressions, the following equation of the garnet-orthopyroxene geothermometer (Eq. 32; see row 32 in Tab. 6) has been derived by Perchuk et al. (1985) to estimate the physical and geodynamic conditions of metamorphism of the Precambrian granulites of the Aldan Shield, eastern Siberia, on the basis of analytical, petrological and experimental data:

$$
\begin{aligned}
& T_{\mathrm{P} 85}^{\mathrm{Grt}-\mathrm{Opx}}\left({ }^{\circ} \mathrm{C}\right)= \frac{4766+2533\left(X_{\mathrm{Fs}}^{\mathrm{Opx}}-X_{\mathrm{En}}^{\mathrm{Opp}}\right)-5214 X_{\mathrm{Al}}^{\mathrm{Opx}}}{1.9872 \ln K_{D}+2.65+1.86\left(X_{\mathrm{Fs}}^{\mathrm{Opx}}-X_{\mathrm{En}}^{\mathrm{O} p}\right)+1.242 X_{\mathrm{Ca}}^{\mathrm{Git}}} \\
& \frac{+5704 X_{\mathrm{Ca}}^{\mathrm{Git}}+23 P(\mathrm{kbar})}{1.9872 \ln K_{D}+2.65+1.86\left(X_{\mathrm{Fs}}^{\mathrm{Opx}}-X_{\mathrm{En}}^{\mathrm{O} \mathrm{px}}\right)+1.242 X_{\mathrm{Ca}}^{\mathrm{Git}}}-273.15
\end{aligned}
$$

where $K_{\mathrm{D}}=\left(X_{\mathrm{Fe}}^{\mathrm{Git}} / X_{\mathrm{En}}^{\mathrm{Opx}}\right) /\left(X_{\mathrm{Mg}}^{\mathrm{Git}} / X_{\mathrm{Fs}}^{\mathrm{O} \mathrm{px}}\right), X_{\mathrm{Fs}}^{\mathrm{Opx}}=\mathrm{Fe} /(\mathrm{Fe}+\mathrm{Mg}+0.5 \mathrm{Al})$, $X_{\mathrm{En}}^{\mathrm{Opx}}=\mathrm{Mg} /(\mathrm{Fe}+\mathrm{Mg}+0.5 \mathrm{Al})$ and $X_{\mathrm{Al}}^{\mathrm{Opx}}=1-X_{\mathrm{Fs}}^{\mathrm{Opx}}-X_{\mathrm{En}}^{\mathrm{Opx}}$.

Lee and Ganguly (1988) carried out an experimental study to determine the $\mathrm{Fe}-\mathrm{Mg}$ fractionation between coexisting garnet and orthopyroxene at 20-45 kbar, $975-1400{ }^{\circ} \mathrm{C}$, and the effect of iron on alumina solubility in orthopyroxene at $25 \mathrm{kbar}, 1200^{\circ} \mathrm{C}$, and $20 \mathrm{kbar}$, $975^{\circ} \mathrm{C}$ in the $\mathrm{FeO}-\mathrm{MgO}-\mathrm{Al}_{2} \mathrm{O}_{3}-\mathrm{SiO}_{2}$ system. Using their own experimental data, Lee and Ganguly (1988) formulated a revised version of their previous (i.e. Lee and Ganguly 1984) garnet-orthopyroxene exchange geothermometer (see row 33 in Tab. 6), applicable to a wide variety of natural garnet-orthopyroxene assemblages: 
Tab. 6 Garnet-orthopyroxene geothermometer estimations by WinGrt program

\begin{tabular}{|c|c|c|c|c|c|}
\hline \multicolumn{2}{|c|}{ Row } & \multirow{2}{*}{$\frac{\text { SG1 }}{38.500}$} & \multirow{2}{*}{$\begin{array}{r}\text { SG2 } \\
38.150\end{array}$} & \multirow{2}{*}{$\begin{array}{r}\text { SG3 } \\
37.800\end{array}$} & \multirow{2}{*}{$\begin{array}{r}\text { SG4 } \\
38.100\end{array}$} \\
\hline 1 & $\mathrm{SiO}_{2}$ & & & & \\
\hline 2 & $\mathrm{TiO}_{2}$ & 0.000 & 0.030 & 0.050 & 0.060 \\
\hline 3 & $\mathrm{Al}_{2} \mathrm{O}_{3}$ & 21.210 & 21.840 & 21.460 & 21.740 \\
\hline 4 & $\mathrm{Cr}_{2} \mathrm{O}_{3}$ & 0.010 & 0.120 & 0.000 & 0.120 \\
\hline 5 & $\mathrm{FeO}$ & 30.940 & 29.980 & 33.790 & 29.740 \\
\hline 6 & $\mathrm{MgO}$ & 6.850 & 6.830 & 4.120 & 7.620 \\
\hline 7 & $\mathrm{MnO}$ & 0.720 & 0.890 & 1.280 & 0.640 \\
\hline 8 & $\mathrm{CaO}$ & 1.770 & 2.760 & 2.890 & 1.890 \\
\hline 9 & $\mathrm{Na}_{2} \mathrm{O}$ & 0.030 & 0.020 & 0.030 & 0.040 \\
\hline 10 & $\sum($ wt. \%) & 100.030 & 100.620 & 101.420 & 99.950 \\
\hline \multicolumn{6}{|c|}{ Garnet recalculation based on 8 cations $(a p f u)$} \\
\hline 11 & $\mathrm{Si}$ & 3.015 & 2.963 & 2.971 & 2.967 \\
\hline 12 & $\mathrm{Ti}$ & 0.000 & 0.002 & 0.003 & 0.004 \\
\hline 13 & $\mathrm{Al}$ & 1.958 & 1.999 & 1.988 & 1.995 \\
\hline 14 & $\mathrm{Cr}$ & 0.001 & 0.007 & 0.000 & 0.007 \\
\hline 15 & $\mathrm{Fe}^{3+}$ & 0.016 & 0.068 & 0.068 & 0.063 \\
\hline 16 & $\mathrm{Fe}^{2+}$ & 2.010 & 1.880 & 2.154 & 1.874 \\
\hline 17 & $\mathrm{Mg}$ & 0.800 & 0.791 & 0.483 & 0.885 \\
\hline 18 & $\mathrm{Mn}$ & 0.048 & 0.059 & 0.085 & 0.042 \\
\hline 19 & $\mathrm{Ca}$ & 0.149 & 0.230 & 0.243 & 0.158 \\
\hline 20 & $\mathrm{Na}$ & 0.005 & 0.003 & 0.005 & 0.006 \\
\hline 21 & $\sum$ cations & 8.000 & 8.000 & 8.000 & 8.000 \\
\hline Garn & & Alm & Alm & Alm & Alm \\
\hline \multicolumn{6}{|c|}{ Input orthopyroxene cations $(a p f u)$ and pressure (kbar) for geothermometer estimations } \\
\hline 22 & $\mathrm{Al}_{\text {tot }}$ & 0.176 & 0.114 & 0.107 & 0.115 \\
\hline 23 & $\mathrm{Fe}_{\text {tot }}$ & 0.891 & 0.906 & 1.173 & 0.858 \\
\hline 24 & $\mathrm{Fe}^{2+}$ & 0.891 & 0.906 & 1.173 & 0.858 \\
\hline 25 & $\mathrm{Mg}$ & 0.974 & 1.008 & 0.772 & 1.097 \\
\hline 26 & $P$ (kbar) & 7 & 7 & 7 & 7 \\
\hline \multicolumn{6}{|c|}{ Garnet-orthopyroxene geothermometers $\left({ }^{\circ} \mathrm{C}\right)$} \\
\hline 27 & $T 1$ & 814 & 876 & 920 & 781 \\
\hline 28 & $T 2$ & 979 & 1016 & 920 & 993 \\
\hline 29 & $T 3$ & 727 & 757 & 707 & 736 \\
\hline 30 & $T 4$ & 810 & 854 & 792 & 819 \\
\hline 31 & $T 5$ & 831 & 869 & 811 & 843 \\
\hline 32 & $T 6$ & 776 & 837 & 822 & 795 \\
\hline 33 & $T 7$ & 795 & 835 & 775 & 807 \\
\hline 34 & $T 8$ & 713 & 764 & 744 & 734 \\
\hline 35 & $T 9$ & 788 & 822 & 789 & 794 \\
\hline 36 & $T 10$ & 801 & 850 & 810 & 817 \\
\hline
\end{tabular}

Notes: The formulae were recalculated to 12 anions and 8 cations; $\mathrm{Fe}^{3+}$ and $\mathrm{Fe}^{2+}$ estimations from total FeO (wt. \%) contents were carried out on the basis of stoichiometric constraints using Droop's (1987) method; sample numbers SG 1to SG4 from Sen and Bhattacharya (1984);

Alm = almandine; Geothermometers T1 by Dahl (1980), T2 by Raith et al. (1983), T3 by Harley (1984), T4 by Sen and Bhattacharya (1984), T5 by Lee and Ganguly (1984), T6 by Perchuk et al. (1985), 77 by Lee and Ganguly (1988), T8 by Perchuk and Lavrent'eva (1990), T9 by Bhattacharya et al. (1991), T10 by Lal (1993)

$$
T_{\mathrm{LG} 88}^{\mathrm{Grt}-\mathrm{Opx}}\left({ }^{\circ} \mathrm{C}\right)=\frac{1981+1509.66\left(X_{\mathrm{Ca}}^{\mathrm{Grt}}-X_{\mathrm{Mn}}^{\mathrm{Git}}\right)+11.91 P(\mathrm{kbar})}{\ln K_{D}+0.97}
$$

Based on the experimental data at temperatures of 700 and $800^{\circ} \mathrm{C}$ over a wide range of pressures (6-30 kbar) for the orthopyroxene-garnet $\mathrm{Fe}^{2+}-\mathrm{Mg}$ exchange reaction, Perchuk and Lavrent'eva (1990) derived a new garnet-orthopyroxene geothermometer (see row 34 in Tab. 6) in the third iteration of the non-ideal nature of the $\mathrm{Fe}-\mathrm{Mg}-\mathrm{Al}$ solid solution in orthopyroxene, as well as taking into account the variable content of $\mathrm{Ca}$ in garnet and $\mathrm{Al}$ in orthopyroxene: 


$$
\begin{aligned}
& T_{\mathrm{PL} 90}^{\mathrm{Grt}-\mathrm{Opx}}\left({ }^{\circ} \mathrm{C}\right)=\frac{4066-347\left(X_{\mathrm{En}}^{\mathrm{Opx}}-X_{\mathrm{Fs}}^{\mathrm{Opx}}\right)-17484 X_{\mathrm{Al}}^{\mathrm{Opx}}}{\mathrm{A}} \\
& \frac{+5769 X_{\mathrm{Ca}}^{\mathrm{Grt}}+23.42 P(\mathrm{kbar})}{\mathrm{A}}-273.15
\end{aligned}
$$

where $\mathrm{A}=1.987 \ln K_{D}+2.143+0.0929\left(X_{\mathrm{En}}^{\mathrm{Opx}}-X_{\mathrm{Fs}}^{\mathrm{Opx}}\right)$ $-12.8994 X_{\mathrm{Al}}^{\mathrm{Opx}}+3.846 X_{\mathrm{Ca}}^{\mathrm{Grt}}$.

Using the retrieved data combined with the known thermochemical parameters for the pyrope-grossular and almandine-grossular binaries, Bhattacharya et al. (1991) published a refined formulation of the garnet-orthopyroxene geothermometer (see row 35 in Tab. 6) based exclusively on calorimetric determinations and high-quality phase-equilibrium data:

$$
\begin{aligned}
& T_{\mathrm{B} 91}^{\mathrm{Grt}-\mathrm{Opx}}\left({ }^{\circ} \mathrm{C}\right)=\frac{1611+21 P(\mathrm{kbar})+906 X_{\mathrm{Ca}}^{\mathrm{Grt}}+\mathrm{A}}{\ln K_{D}+0.796} \\
& \frac{+477\left(2 X_{\mathrm{En}}^{\mathrm{Opx}}-1\right)}{\ln K_{D}+0.796}-273.15
\end{aligned}
$$

where $\mathrm{A}=-1220 X_{\mathrm{Fe}}^{\mathrm{Grt}} X_{\mathrm{Mg}}^{\mathrm{Grt}} X_{\mathrm{Ca}}^{\mathrm{Grt}}\left(X_{\mathrm{Mg}}^{\mathrm{Grt}}-X_{\mathrm{Fe}}^{\mathrm{Grt}}\right)-136\left(X_{\mathrm{Mg}}^{\mathrm{Grt}}\right)^{2}$ $+746\left(X_{\mathrm{Fe}}^{\mathrm{Git}}\right)^{2}$.

According to Bhattacharya et al. (1991), Eq. 35 can be used to estimate the temperatures from garnet-orthopyroxene pairs for $X_{\mathrm{Ca}}^{\mathrm{Grt}} \leq 30 \mathrm{~mol} \%, X_{\mathrm{Mn}}^{\mathrm{Grt}} \leq 0.05 \mathrm{~mol} \%$ and pressures $<15$ kbar.

Lal (1993) refined the calibration of the $\mathrm{Fe}^{2+}-\mathrm{Mg}$ exchange garnet-orthopyroxene geothermometer (see row 36 in Tab. 6) using the large dataset for the $\mathrm{FeO}-$ $\mathrm{MgO}-\mathrm{AI}_{2} \mathrm{O}_{3}-\mathrm{SiO}_{2}$ and $\mathrm{CaO}-\mathrm{FeO}-\mathrm{MgO}-\mathrm{Al}_{2} \mathrm{O}_{3}-\mathrm{SiO}_{2}-\mathrm{H}_{2} \mathrm{O}$ systems in a wide range of pressure and temperature conditions $\left(700-1400^{\circ} \mathrm{C}\right.$ and $\left.5-50 \mathrm{kbar}\right)$ :

$$
\begin{aligned}
& T_{\mathrm{L} 93}^{\mathrm{Grt}-\mathrm{Opx}}\left({ }^{\circ} \mathrm{C}\right)=\frac{3367+21 P-1(\mathrm{kbar}) 24 \mathrm{~A}-948\left(X_{\mathrm{Fs}}^{\mathrm{Opx}}-X_{\mathrm{En}}^{\mathrm{Opx}}\right)}{1.987 \ln K_{D}+1.634+\mathrm{B}-0.34\left(X_{\mathrm{Fs}}^{\mathrm{Opx}}-X_{\mathrm{En}}^{\mathrm{Opx}}\right)} \\
& \frac{-1950 X_{\mathrm{Al}}^{\mathrm{Opx}}}{1.987 \ln K_{D}+1.634+\mathrm{B}-0.34\left(X_{\mathrm{Fs}}^{\mathrm{Opx}}-X_{\mathrm{En}}^{\mathrm{Opx}}\right)}-273.15
\end{aligned}
$$

where

$$
\begin{aligned}
& \mathrm{A}=-1256 X_{\mathrm{Mg}}^{\mathrm{Grt}}-2880\left(X_{\mathrm{Fe}}^{\mathrm{Grt}}\right)^{2}+8272 X_{\mathrm{Mg}}^{\mathrm{Grt}} X_{\mathrm{Fe}}^{\mathrm{Grt}}+812 X_{\mathrm{Ca}}^{\mathrm{Grt}} \\
& \times\left(X_{\mathrm{Mg}}^{\mathrm{Grt}}-X_{\mathrm{Fe}}^{\mathrm{Grt}}\right)+90\left(X_{\mathrm{Ca}}^{\mathrm{Grt}}\right)^{2}-2340 X_{\mathrm{Ca}}^{\mathrm{Grt}}\left(X_{\mathrm{Fe}}^{\mathrm{Grt}}+X_{\mathrm{Mg}}^{\mathrm{Grtt}}\right) \\
& -3047 X_{\mathrm{Mg}}^{\mathrm{Grt}} X_{\mathrm{Ca}}^{\mathrm{Grt}}-1813 X_{\mathrm{Fe}}^{\mathrm{Grt}} X_{\mathrm{Ca}}^{\mathrm{Grt}}-4498 X_{\mathrm{Ca}}^{\mathrm{Grt}}\left(X_{\mathrm{Fe}}^{\mathrm{Grt}}-X_{\mathrm{Mg}}^{\mathrm{Grt}}\right)
\end{aligned}
$$

and

$$
\begin{aligned}
& \mathrm{B}=\left(X_{\mathrm{Mg}}^{\mathrm{Grt}}\right)^{2}+1.7\left(X_{\mathrm{Fe}}^{\mathrm{Grt}}\right)^{2}-5.4 X_{\mathrm{Fe}}^{\mathrm{Grt}} X_{\mathrm{Mg}}^{\mathrm{Grt}}-0.35 X_{\mathrm{Ca}}^{\mathrm{Grt}} \\
& \times\left(X_{\mathrm{Mg}}^{\mathrm{Grt}}-X_{\mathrm{Fe}}^{\mathrm{Grt}}\right)+1.5\left(X_{\mathrm{Ca}}^{\mathrm{Grt}}\right)^{2}+1.666 X_{\mathrm{Ca}}^{\mathrm{Grt}}\left(X_{\mathrm{Fe}}^{\mathrm{Grt}}+X_{\mathrm{Mg}}^{\mathrm{Grt}}\right) \\
& +0.332 X_{\mathrm{Fe}}^{\mathrm{Grt}} X_{\mathrm{Ca}}^{\mathrm{Grt}}+1.516 X_{\mathrm{Ca}}^{\mathrm{Grt}}\left(X_{\mathrm{Fe}}^{\mathrm{Grt}}-X_{\mathrm{Mg}}^{\mathrm{Grt}}\right) .
\end{aligned}
$$

According to Lal (1993), Eq. 36 is applicable to the garnet-orthopyroxene pairs from crustal rocks including garnet with low Mn content $\left(X_{\mathrm{Mn}}^{\mathrm{Grt}}<5 \mathrm{~mol} \%\right.$ ), as well as mantle-derived rocks with a low $\mathrm{Cr}_{2} \mathrm{O}_{3}$ content in garnet $(<5$ wt. $\%)$.

\subsection{Garnet in provenance studies}

Parallel to the development of microanalysis equipment, a wide group of detrital minerals such as pyroxene, amphibole, epidote, tourmaline, and garnet were considered as potential provenance indicators in sedimentary environments. Although heavy minerals have been used in a variety of provenance studies, some of them are sensitive to diagenesis and many species suffer from the dissolution process known as intrastratal solution (Morton 1985). For example pyroxene, amphibole and epidote group minerals are moderately or extremely susceptible to intrastratal solution under both deep burial and weathering conditions. Even though composition of tourmaline, as a refractory mineral, may be used in provenance studies (e.g. Henry and Dutrow 2018), garnet minerals remain the most suitable group to determine the provenance of sediments (Morton 1985).

Because garnet composition is a function of $P-T$ conditions, and garnet is comparatively stable during surface weathering, transport, and deep burial, several geochemical discrimination schemes have been developed and applied as a tool in sedimentary provenance analysis (Schönig et al. 2018). Wright (1938) made the first attempt to classify garnets from different host-rock types in ternary (almandine + spessartine)-pyrope-grossular (mol. \%) discrimination diagram (Fig. 5a), including granites and granite pegmatites, biotite schists, amphibolites and eclogites. However, there is a strong overlap between garnets from biotite schists and amphibolites, and between amphibolites and eclogites (Preston et al. 2002; Mange and Morton 2007). The first insight into the potential of garnet geochemistry as a provenance indicator by using electron-microprobe analysis provided the study of Middle Jurassic sandstones from the North Sea by Morton (1985). Ternary garnet classification diagram (Fig. 5b) using the molecular proportions of pyrope-spessartinegrossular, but without involving the Fe-component, was proposed by Teraoka et al. $(1997,1998)$ for amphibolites, granulites, and eclogite-facies rocks. Similarly, Morton et al. (2004) used the (almandine + spessartine)-pyropegrossular projection (Fig. 5c) of garnet compositions from Scottish and Norwegian basement terrains, but with the newly defined A, B and C fields, and suggested that garnet compositions can be directly tied back to the adjacent source areas. Modified version of this diagram (Fig. 5d) with an additional D field was introduced by Mange and Morton (2007). Aubrecht et al. (2009) proposed two new ternary classification diagrams (Fig. 5e-f) using molecular proportions of almandine-pyrope-grossular for (ultra-) 

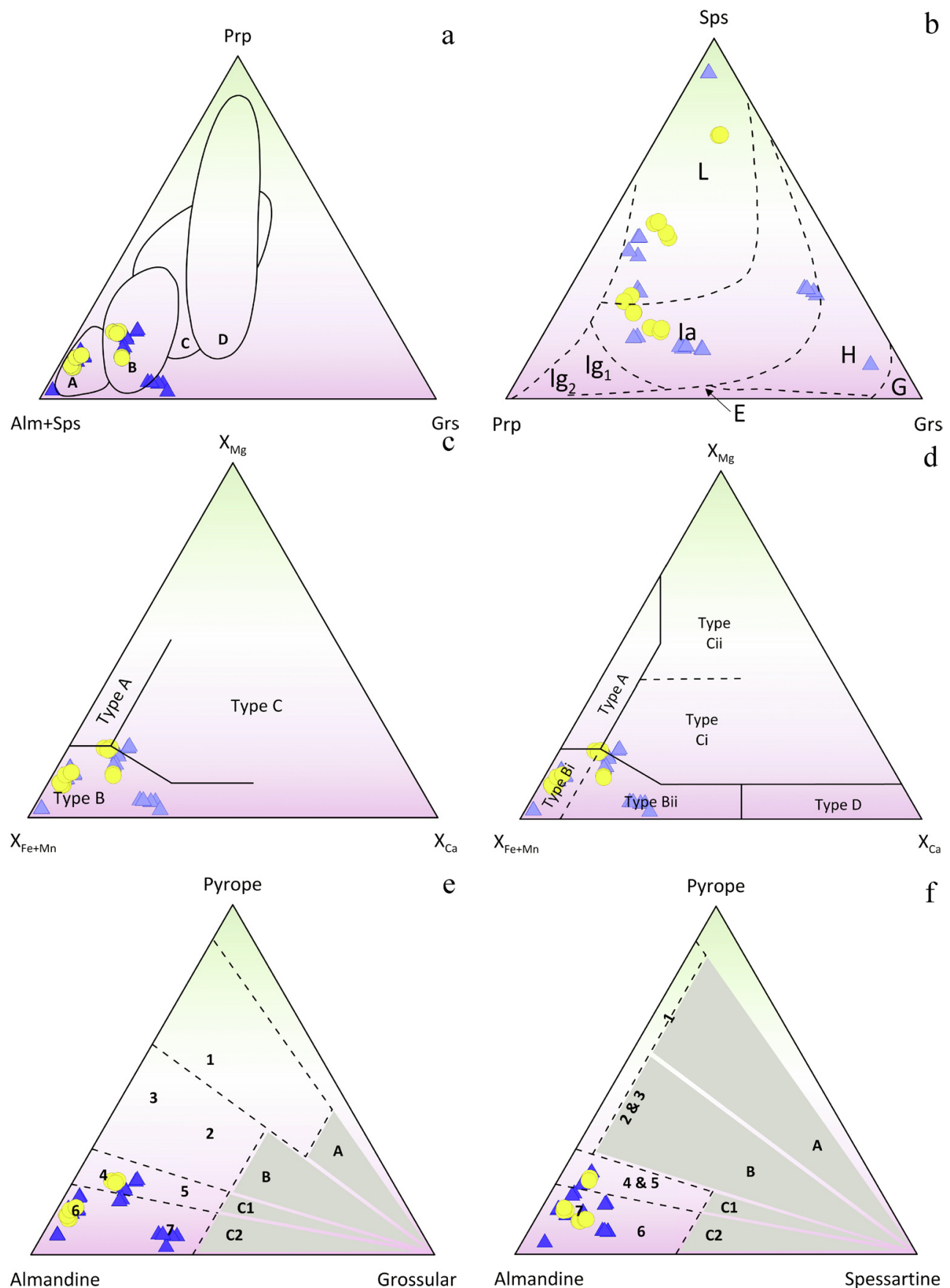

Almandine

Grossular

Almandine

Fig. 5 Ternary discrimination diagrams using molar fractions (\%) of garnet end-members in provenance study of sediments. a $-($ Almandine + spessartine)- pyrope-grossular. A = granites and granite pegmatites, $\mathrm{B}=$ biotite schists, $\mathrm{C}=$ amphibolites, $\mathrm{D}=$ eclogites (Wright 1938$)$. $\mathbf{b}-\mathrm{Pyrope}-$ spessartine-grossular. $\mathrm{L}=$ low $P-T$, la $=$ intermediate $P-T$ (up to amphibolite facies), $\mathrm{H}=$ high $P-T, \lg _{1}, \lg _{2}=$ intermediate $P-T$ (granulite facies), $\mathrm{E}=$ eclogite, $\mathrm{G}=$ grandite garnets (Teraoka et al. 1997, 1998). $\mathbf{c}-\left(X_{\mathrm{Fe}}+X_{\mathrm{Mn}}\right)-X_{\mathrm{Mg}}-X_{\mathrm{Ca}} . \mathrm{A}=$ low Ca, high $\mathrm{Mg}, \mathrm{B}=$ low $\mathrm{Mg}$, variable Ca, $\mathrm{C}=$ high $\mathrm{Ca}$, high $\mathrm{Mg}$ (Morton et al. 2004). d $-\left(X_{\mathrm{Fe}}+X_{\mathrm{Mn}}\right)-X_{\mathrm{Mg}}-X_{\mathrm{Ca}}$. Garnets from: $\mathrm{A}=$ mainly high-grade granulite-facies metasediments or charnockites and intermediate felsic igneous rocks, $\mathrm{B}=$ amphibolite-facies metasedimentary rocks, $\mathrm{Bi}=$ intermediate to felsic igneous rocks, $\mathrm{Ci}=$ mainly high-grade mafic rocks, $\mathrm{Cii}=$ ultramafics with high $\mathrm{Mg}$ (pyroxenites and peridotites), $\mathrm{D}=$ metasomatic rocks, very low-grade metabasic rocks and ultrahigh-temperature calc-silicate granulites (Mange and Morton 2007). e - Almandine-pyrope-grossular and $\mathbf{f}$ - Almandine-pyrope-spessartine. Garnets from: $\mathrm{A}=$ high-pressure (HP) to ultrahigh-pressure (UHP) rocks, $\mathrm{B}=$ eclogite- and granulite-facies rocks, $\mathrm{C} 1=$ higher amphibolite- to granulite-facies rocks, $\mathrm{C} 2=$ amphibolite-facies rocks but also any other rocks such as blueschists, skarns, serpentinites and igneous rocks. Garnets derived from: $1=\mathrm{UHP}$ eclogites or garnet peridotites, 2 = HP eclogites and HP mafic granulites, $3=$ felsic-intermediate granulites, $4=$ gneisses metamorphosed under P-T conditions transitional between granulite- and amphibolite-facies metamorphism, $5=$ amphibolites metamorphosed under $\mathrm{P}-\mathrm{T}$ conditions transitional between granulite- and amphibolite-facies metamorphism, $6=$ amphibolite-facies gneisses, $7=$ amphibolites proper (Aubrecht et al. 2009). Garnet analyses in all plots are taken from Suggate and Hall (2014). Triangles and circles correspond to tables 4 and 5 in Suggate and Hall (2014), respectively. 


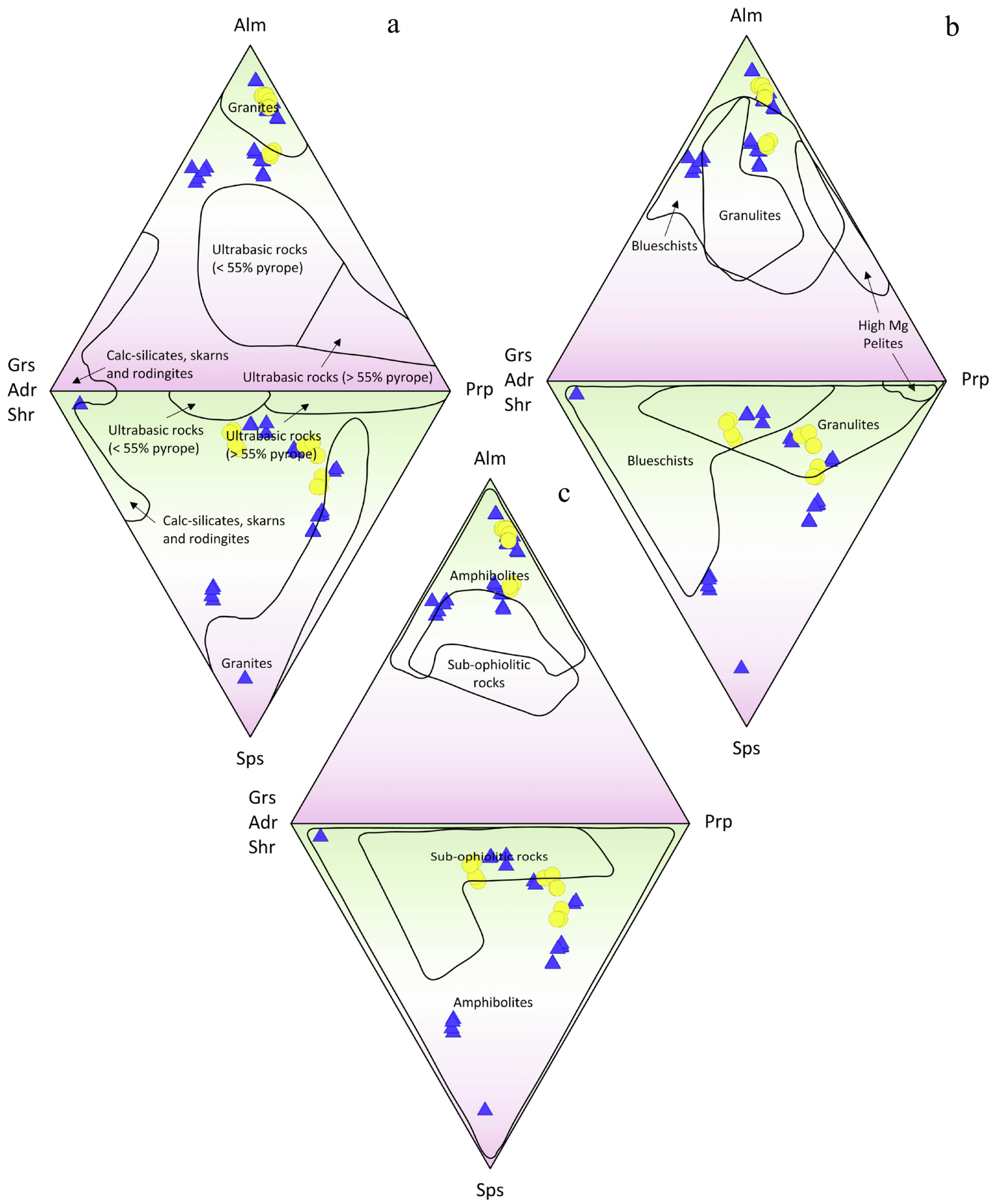

Fig. 6 Ternary plots based on garnet end-members discriminating garnets with different protoliths (Suggate and Hall 2014). a - Ultramafic rocks (peridotites, eclogites and kimberlites), granites, calc-silicates, skarns and rodingites. b-Granulites, granulite-facies high-Mg pelites, and blueschists. c-Amphibolites and metabasic sub-ophiolitic rocks. Triangles and circles correspond to tables 4 and 5 in Suggate and Hall (2014), respectively.

high-pressure metamorphic rocks and almandine-pyropespessartine for granulite- and amphibolite-facies rocks (after Méres 2008).
Suggate and Hall (2014) presented a new approach to garnet discrimination that invokes two ternary diagrams (Fig. 6) with almandine and spessartine apices, and a 
common axis (grossular + andradite + schorlomite)-pyrope. Their scheme requires a stepwise separation of garnets of specific composition. Hence, garnets with an uncommon content of $\mathrm{Y}_{2} \mathrm{O}_{3}, \mathrm{~V}_{2} \mathrm{O}_{3}$, and $\mathrm{ZrO}_{2}$ are first removed and then those with high $\mathrm{TiO}_{2}$ are separated in their discrimination diagrams. This type of projection enables to group garnets derived from ores, skarns, mafic pyroclastic rocks, and nepheline syenites. In a next step, another group of garnets with high pyrope and uvarovite end-member compositions derived from ultramafic rocks is distinguished. Following the second step, all the remaining garnets, i.e., those from amphibolite-, granulite-, and eclogite-facies rocks, are plotted in ternary diagrams with almandine and spessartine apices. These garnets may overlap, but many mafic eclogites can be distinguished by their low spessartine content (Krippner et al. 2014; Suggate and Hall 2014).

\section{Summary and availability of the program}

WinGrt is a user-friendly program, which is specially developed for personal computers running in the Windows operating system to estimate and classify the garnet supergroup mineral analyses obtained by electron-microprobe or wet-chemical analyses. The program calculates multiple (up to 300) garnet supergroup analyses on the basis of 8 cations and 12 anions. Following the procedure by Droop (1987), WinGrt estimates the $\mathrm{Fe}^{2+}$ and $\mathrm{Fe}^{3+}$ and, if necessary, $\mathrm{Mn}^{3+}(a p f u)$ contents from electron-microprobe analyses using the stoichiometric constraints. It also provides users a large number of geothermometers to determine the temperature conditions based on the $\mathrm{Fe}^{2+}-$ $\mathrm{Mg}$ exchange reactions of garnet-biotite, garnet-clinopyroxene, and garnet-orthopyroxene pairs in greenschist- to eclogite-facies metamorphic rocks. The program yields end-member molecular proportions using various normalization schemes to plot ternary discrimination diagrams in sedimentary provenance analysis by using the Golden Software's Grapher software.

WinGrt generates two main windows. The first (i.e. Start-up/Data Entry Screen), with several pull-down menus and equivalent shortcuts, enables to edit garnet analyses (wt. \%) and recalculated specific input biotite, clinopyroxene and orthopyroxene cation values such as $\mathrm{Fe}^{2+}, \mathrm{Mg}, \mathrm{Al}^{\mathrm{VI}}, \mathrm{Ti}, \mathrm{Fe}_{\text {tot }}$ and $\mathrm{Al}_{\text {tot }}(a p f u)$ into the data entry section as well as to carry out necessary arrangements for a desired calculation scheme. By clicking the Calculate icon (i.e. $\sum$ ) in the Data Entry Screen, all calculated parameters are displayed in the second window (i.e. Calculation Screen). WinGrt reports the output in a tabulated form with columns numbered from 1 to 187 in the Calculation Screen window. Garnet-only-related pa- rameters are listed at columns 1-140. Calculated garnetbiotite, garnet-clinopyroxene and garnet-orthopyroxene geothermometers with input parameters are displayed by light blue, green and pink colours in columns 142-156, 158-172 and 174-187 of the Calculation Screen, as well as in an Output Excel file. The results in the Calculation Screen can be exported to Microsoft ${ }^{\circledR}$ Excel file (i.e. Output.xlsx), by clicking the Send Results to Excel File (Output.xlsx) icon or selecting the Send Results to Excel File (Output.xlsx) option from the Excel pull-down menu. Subsequently, this file is opened in Excel by clicking the Open and Edit Excel File (Output.xlsx) icon or selecting the Open Excel File (Output.xlsx) option from the Excel pull-down menu.

WinGrt is a compiled program that consists of a selfextracting setup file including all the necessary support files (i.e. *. dll and ${ }^{\star} . \mathrm{OCx}$ ) for the 32-bit system. During the setup procedure, the program and its associated files (i.e. support files, help file, data files with the extensions of $g s g, x l s, x l s x$ and plot files with the extension of $g r f$ ) are installed into the personal computer (i.e. the directory $C: \backslash$ Program Files $\backslash$ WinGrt) with the Windows XP or later operating systems. An installation of program into a personal computer with the 64-bit operating system may require the msflexgrd adjustment. This procedure is explained in detail in Electronic Supplementary Material (ESM 2). The self-extracting setup file is $14 \mathrm{MB}$ and can be obtained from the journal server or from corresponding author on request.

Acknowledgements. We thank Renata Čopjaková and an anonymous reviewer for their constructive reviews and contributions which improved the quality of manuscript. We also thank handling editor, Jiří Konopásek, and Vojtěch Janoušek, editor-in-chief, for their valuable comments and suggestions on text and program structure.

Electronic supplementary material. Examples of garnet data recalculated and plotted by the WinGrt program, the steps for its execution and self-extracting WinGrt. exe setup file are available online at the Journal web site (http://dx.doi.org/10.3190/jgeosci.303).

\section{References}

AI Y (1994) A revision of the garnet-clinopyroxene $\mathrm{Fe}^{2+}-$ Mg exchange geothermometer. Contrib Mineral Petrol 115: 467-473

ANTAO SM (2014) Schorlomite and morimotoite: what's in a name? Powder Diffr 29: 346-351

Antao SM, Cruickshank LA (2018) Crystal structure refinements of tetragonal $(\mathrm{OH}, \mathrm{F})$-rich spessartine and henritermierite garnets. Acta Cryst B74: 104-114 
Aubrecht R, Méres Š, Sýkora M, Mikus T (2009) Provenance of the detrital garnets and spinels from the Albian sediments of the Czorsztyn Unit (Pieniny Klippen Belt, Western Carpathians, Slovakia). Geol Carpath 60: 463-483

BaXter EF, Caddick MJ, Ague JJ (2013) Garnet: common mineral, uncommonly useful. Elements 9: 415-419

Bhattacharya A, Krishnakumar KR, Raith M, Sen SK (1991) An improved set off $a-X$ parameters for $\mathrm{Fe}-\mathrm{Mg}-$ $\mathrm{Ca}$ garnets and refinements of the orthopyroxene-garnet thermometer and the orthopyroxene-garnet-plagioclasequartz barometer. J Petrol 32: 629-656

Bhattacharya A, Mohanty L, Maji A, Sen SK, Raith M (1992) Non-ideal mixing in the phlogopite-annite binary: constraints from experimental data on $\mathrm{Mg}-\mathrm{Fe}$ partitioning and a reformulation of the biotite-garnet geothermometer. Contrib Mineral Petrol 111: 87-93

Bohlen SR, Essene EJ (1980) Evaluation of coexisting garnet-biotite, garnet-clinopyroxene, and other $\mathrm{Mg}-\mathrm{Fe}$ exchange thermometers in Adirondack granulites: summary. Geol Soc Am Bull 91: 107-109

Bucher K, Grapes R (2011) Petrogenesis of Metamorphic Rocks, $8^{\text {th }}$ Edition. Springer, Berlin, pp 1-428

DAHL PS (1980) The thermal-compositional dependence of $\mathrm{Fe}^{2+}-\mathrm{Mg}$ distributions between coexisting garnet and pyroxene: applications to geothermometry. Amer Miner 65: 854-866

Dasgupta S, Sengupta P, Guha D, Fukuoka M (1991) A refined garnet-biotite $\mathrm{Fe}-\mathrm{Mg}$ exchange geothermometer and its application in amphibolites and granulites. Contrib Mineral Petrol 109: 130-137

Droop GTR (1987) A general equation for estimating $\mathrm{Fe}^{3+}$ concentrations in ferromagnesian silicates and oxides from microprobe analyses using stoichiometric criteria. Mineral Mag 51: 431-435

ElLis DJ, GrEen DH (1979) An experimental study of the effect of Ca upon garnet-clinopyroxene $\mathrm{Fe}-\mathrm{Mg}$ exchange equilibria. Contrib Mineral Petrol 71: 13-22

FERRY JM, SPEAR FS (1978) Experimental calibration of the partitioning of $\mathrm{Fe}$ and $\mathrm{Mg}$ between biotite and garnet. Contrib Mineral Petrol 66: 113-117

FRIBERG LM (1989) Garnet stoichiometry program using a Lotus 1-2-3 spreadsheet. Comput Geosci 15: 1169-1172

Galuskina IO, Galuskin EV, Armbruster T, Lazic B, Kusz J, DzierŻanowski P, Gazeev VM, Pertsev NN, Prusik K, Zadov AE, Winiarski A, WrZalik R, GurBANOV AG (2010) Elbrusite-(Zr) - a new uranian garnet from the Upper Chegem Caldera, Kabardino-Balkaria, northern Caucasus, Russia. Amer Miner 95: 1172-1181

Galuskina IO, Galuskin EV, Prusik K, Gazeev VM, Pertsev NN, Dzierżanowski P (2013) Irinarassite $\mathrm{Ca}_{3} \mathrm{Sn}_{2} \mathrm{SiAl}_{2} \mathrm{O}_{12}$ - new garnet from the Upper Chegem Caldera, northern Caucasus, Kabardino-Balkaria, Russia. Mineral Mag 77: 2857-2866
GANGULY J (1979) Garnet and clinopyroxene solid solutions, and geothermometry based on $\mathrm{Fe}-\mathrm{Mg}$ distribution coefficient. Geochim Cosmochim 43: 1021-1029

Ganguly J, SAXENa SK (1984) Mixing properties of aluminosilicate garnets: constraints from natural and experimental data, and applications to geothermo-barometry. Amer Miner 69: 88-97

Ganguly J, Cheng W, Tirone M (1996) Thermodynamics of aluminosilicate garnet solid solution: new experimental data, an optimized model, and thermometric applications. Contrib Mineral Petrol 126: 137-151

Geiger C (2013) Garnet: a key phase in nature, the laboratory, and technology. Elements 9: 447-452

Grew ES, Locock AJ, Mills SJ, Galuskina IO, Galuskin EV, HÅlenius U (2013) Nomenclature of the garnet supergroup. Amer Miner 98: 785-811

HACKLER RT, WOOD BJ (1989) Experimental determination of $\mathrm{Fe}$ and $\mathrm{Mg}$ exchange between garnet and olivine and estimation of $\mathrm{Fe}-\mathrm{Mg}$ garnet mixing properties. Amer Miner 74: 994-999

HARLEY SL (1984) An experimental study of the partitioning of $\mathrm{Fe}$ and $\mathrm{Mg}$ between garnet and orthopyroxene. Contrib Mineral Petrol 86: 359-373

HENRY DJ, Dutrow BL (2018) Tourmaline studies through time: contributions to scientific advancements. J Geosci 63: 77-98

Henry DJ, Novák M, Hawthorne FC, Ertl A, Dutrow BL, Uher P, Pezozotta F (2011) Nomenclature of the tourmaline-supergroup minerals. Amer Mineral 96: 895-913

Hodges KV, SPeAR FS (1982) Geothermometry, geobarometry and the $\mathrm{Al}_{2} \mathrm{SiO}_{5}$ triple point at Mt. Moosilauke, New Hampshire. Amer Miner 67: 1118-1134

HOINKES G (1986) Effect of grossular-content in garnet on the partitioning of $\mathrm{Fe}$ and $\mathrm{Mg}$ between garnet and biotite. An empirical investigation on staurolite-zone samples from the Austroalpine Schneeberg Complex. Contrib Mineral Petrol 92: 393-399

Hora JM, Kronz A, Möller-McNett S, Wörner G (2013) An Excel-based tool for evaluating and visualizing geothermobarometry data. Comput Geosci 56: 178-185

INDARES A, MARTIGNOLE J (1985) Biotite-garnet geothermometry in the granulite facies: the influence of Ti and $\mathrm{Al}$ in biotite. Amer Miner 70: 272-278

KnierZinger W, Wagreich M, Kiraly F, LeE EV, Ntaflos T (2019) TETGAR_C: a novel three-dimensional (3D) provenance plot and calculation tool for detrital garnets. J Geosci 64: 127-148

KNOWLES CR (1987) A BASIC program to recast garnet end-members. Comput Geosci 13: 655-658

Krippner A, Meinhold G, Morton AC, von Eynatten H (2014) Evaluation of garnet discrimination diagrams using geochemical data derived from various host rocks. Sediment Geol 306: 36-52 
KroGH EJ (1988) The garnet-clinopyroxene Fe-Mg geothermometer - a reinterpretation of existing experimental data. Contrib Mineral Petrol 99: 44-48

Krogh-Ravna E (2000) The garnet-clinopyroxene $\mathrm{Fe}^{2+}$ $\mathrm{Mg}$ geothermometer: an updated calibration. J Metamorph Geol 18: 211-219

LAL RK (1993) Internally consistent recalibrations of mineral equilibria for geothermobarometry involving garnet-orthopyroxene-plagioclase-quartz assemblages and their application to the South Indian granulites. J Metamorph Geol 11: 855-866

Lanari P, Vidal O, De Andre V, Dubacq B, Lewin E, Grosch EG, Schwartz S (2014) XMapTools: a MATLAB ${ }^{\circ}$-based program for electron microprobe $\mathrm{X}$-ray image processing and geothermobarometry. Comput Geosci 62: 227-240

LeE HY, Ganguly J (1984) Fe, Mg fractionation between garnet and orthopyroxene: experimental data and application. Geological Society of America Abstract No. 52733

LeE HY, GANGuly J (1988) Equilibrium compositions of coexisting garnet and orthopyroxene: experimental determinations in the system $\mathrm{FeO}-\mathrm{MgO}-\mathrm{Al}_{2} \mathrm{O}_{3}-\mathrm{SiO}_{2}$, and applications. J Petrol 29: 93-113

LIU J (1998) Assessment of the garnet-clinopyroxene thermometer. Int Geol Rev 40: 579-608

Locock AJ (2008) An Excel spreadsheet to recast analyses of garnet into end-member components, and a synopsis of the crystal chemistry of natural silicate garnets. Comput Geosci 34: 1769-1780

Locock AJ (2014) An Excel spreadsheet to classify chemical analyses of amphiboles following the IMA 2012 recommendations. Comput Geosci 62: 1-14

Ma C, Krot AN (2014) Hutcheonite, $\mathrm{Ca}_{3} \mathrm{Ti}_{2}\left(\mathrm{SiAl}_{2}\right) \mathrm{O}_{12}$, a new garnet mineral from the Allende meteorite: an alteration phase in a Ca-Al-rich inclusion. Amer Miner 99: 667-670

Mange MA, Morton AC (2007) Geochemistry of heavy minerals. In: MANGE MA, Wright DT (eds) Heavy Minerals in Use. Elsevier, Amsterdam, pp 345-391

MÉRES Š (2008) Garnets - an important information resource about source area and parent rocks of siliciclastic sedimentary rocks. In: JuRKovič L' (ed) Conference "Cambelové dni 2008”, Abstract Book. Comenius University, Bratislava, pp 37-43 (in Slovak with English summary)

Morton AC (1985) A new approach to provenance studies: electron microprobe analysis of detrital garnets from Middle Jurassic sandstones of the northern North Sea. Sedimentology 32: 553-566

Morton A, Hallsworth C, Chalton B (2004) Garnet compositions in Scottish and Norwegian basement terrains: a framework for interpretation of North Sea sandstone provenance. Mar Petrol Geol 21: 393-410

Muhling JR, GRIFFIN BJ (1991) On recasting garnet analyses into end-member molecules - revisited. Comput Geosci 17: 161-170
Munno R, Rossi G, TAdini C (1980) Crystal chemistry of kimzeyite from Stromboli, Aeolian Islands, ltaly. Amer Miner 65: 188-191

NAgashima M, Armbruster T (2012) Palenzonaite, berzeliite, and manganberzeliite: $\left(\mathrm{As}^{5+}, \mathrm{V}^{5+}, \mathrm{Si}^{4+}\right) \mathrm{O}_{4}$ tetrahedra in garnet structures. Mineral Mag 76: 1081-1097

NAKAMURA D (2009) A new formulation of garnet-clinopyroxene geothermometer based on accumulation and statistical analysis of a large experimental data set. J Metamorph Geol 27: 495-508

NAKAMURA D, HiraJima T (2005) Experimental evaluation of garnet-clinopyroxene geothermometry as applied to eclogites. Contrib Mineral Petrol 150: 581-588

PERCHUK LL, LAVRENT'EVA IV (1983) Experimental investigation of exchange equilibria in the system cordierite-garnet-biotite. In: SAXENA SK (ed) Kinetics and Equilibrium in Mineral Reactions. Springer, New York, pp 1-273

Perchuk LL, LaVrent'Eva IV (1990) Garnet-orthopyroxene and garnet-amphibole geothermobarometry: experimental data and thermodynamics. Int Geol Rev 32: 486-507

Perchuk LL, Aranovich LYa, Podlesskit KK, Lavrent'eva IV, Gerasimov VyU, Fed' kin VV, Kitsul VI, Karsakov LP, BERDNIKOV NV (1985) Precambrian granulites of the Aldan shield, eastern Siberia, USSR. J Metamorph Geol 3: 265-310

Powell R (1985) Regression diagnostics and robust regression in geothermometer/geobarometer calibration: the garnet-clinopyroxene geothermometer revisited. J Metamorph Geol 3: 231-243

Preston J, Hartley A, Mange-Rajetzky M, Hole M, May G, Buck S, Vaughan L (2002) The provenance of Triassic continental sandstones from the Beryl Field, northern North Sea: mineralogical, geochemical, and sedimentological constraints. J Sediment Res 72: 18-29

PUTIRKA K (2008) Thermometers and barometers for volcanic systems. In: Putirka KD, TePley III FJ (eds) Minerals, Inclusions And Volcanic Processes. Mineralogical Society of America and Geochemical Society Reviews in Mineralogy and Geochemistry 69, Washington, pp 61-120

RAITH M, RAASE P, ACKERMAND D, LAL RK (1983) Regional geothermobarometry in the granulite facies terrane of South India. Trans Roy Soc Edinb, Earth Sci 73: 221-244

Schingaro E, Lacalamita M, Mesto E, Ventruti G, Pedrazzi G, Ottolini L, Scordari F (2016) Crystal chemistry and light elements analysis of Ti-rich garnets. Amer Miner 101: 371-384

RÅHEIM A, GREEN DH (1974) Experimental determination of the temperature and pressure dependence of the $\mathrm{Fe}-\mathrm{Mg}$ partition coefficient for coexisting garnet and clinopyroxene. Contrib Mineral Petrol 48: 179-203

Raith M, RaAse P, ACKermand D, LAI RK (1983) Regional geothermobarometry in the granulite facies terrane of South India. Trans Roy Soc Edinb, Earth Sci 73: 221-244 
Rickwood PC (1968) On recasting analyses of garnet into end-member molecules. Contrib Mineral Petrol 18: 175-198

Schönig J, Meinhold G, Von Eynattena H, LünsDORF NK (2018) Provenance information recorded by mineral inclusions in detrital garnet. Sediment Geol 376: 32-49

SEN SK, BhatTACHARYA A (1984) An orthopyroxene-garnet thermometer and its application to the Madras charnockites. Contrib Mineral Petrol 88: 64-71

SugGate SM, Hall R (2014) Using detrital garnet compositions to determine provenance: a new compositional database and procedure. In: ScOTt RA, SMYTH HR, Morton AC, Richardson N (eds) Sediment Provenance Studies in Hydrocarbon Exploration and Production. Geological Society of London Special Publications 386, pp 373-393

Teraoka Y, Suzuki M, Hayashi T, Kawakami K (1997) Detrital garnets from Paleozoic and Mesozoic sandstones in the Onogawa area, East Kyushu, southwest Japan. Bull Fac Sch Educ, Hiroshima University, Part II 19: 87-101 (in Japanese with English abstract)

Teraoka Y, Suzuki M, KawaKami K (1998) Provenance of Cretaceous and Paleogene sediments in the Median Zone of southwest Japan. Bull Geol Soc Japan 49: 395-411 (in Japanese with English abstract)

THOEnEn T (1989) A Comparative Study of Garnet-Biotite Geothermometers. Unpublished PhD. thesis, University of Basel, pp 1-118

Thompson AB (1976) Mineral reactions in pelitic rocks: II. Calculation of some $\mathrm{P}-\mathrm{T}-\mathrm{X}(\mathrm{Fe}-\mathrm{Mg})$ phase relations. Amer J Sci 276: 425-454

Tolosana-Delgado R, Von Eynatten H, Krippner A, Meinhold G (2018) A multivariate discrimination scheme of detrital garnet chemistry for use in sedimentary provenance analysis. Sediment Geol 375: 14-26
Wright WI (1938) The composition and occurrence of garnets. Amer Miner 23: 436-449

YANG J (1991) A new scheme for calculating mineral endmembers with reference to clinopyroxene and garnet. Acta Geol Sin 65: 360-366

YAVUZ F (2001) PYROX: a computer program for the IMA pyroxene classification and calculation scheme. Comput Geosci 27: 97-107

YAVUZ F (2003a) Evaluating micas in petrologic and metallogenic aspect: I - definitions and structure of the computer program $\mathrm{MICA}^{+}$. Comput Geosci 29: 1203-1213

YAVUZ F (2003b) Evaluating micas in petrologic and metallogenic aspect: II - applications using the computer program $\mathrm{MICA}^{+}$. Comput Geosci 29: 1215-1228

YAVUZ F (2007) WinAmphcal: a Windows program for the IMA-04 amphibole classification. Geochem Geophys Geosyst 8: Q01004, DOI 10.1029/2006GC001391

YAVUZ F (2013) WinPyrox: a Windows program for pyroxene calculation classification and thermobarometry. Amer Miner 98: 1338-1359

YAVUZ F, DöNER Z (2017) WinAmptb: a Windows program for calcic amphibole thermobarometry. Period Mineral 86: $135-167$

YAVuz F, Yildirim DK (2018) A Windows program for calculation and classification of epidote-supergroup minerals. Period Mineral 87: 269-285

Yavuz F, Yavuz V, SASMAz A (2006) WinClastour - a Visual Basic program for tourmaline formula calculation and classification. Comput Geosci 32: 1156-1168

Yavuz F, Karakaya N, Yildirim DK, Karakaya MC, KumRAL M (2014) A Windows program for calculation and classification of tourmaline-supergroup (IMA-2011). Comput Geosci 63: 70-87

Yavuz F, Kumral M, Karakaya N, Karakaya MC, YildirIM DK (2015) A Windows program for chlorite calculation and classification. Comput Geosci 81: 101-113 